\title{
Effect of Heat Release on Streamwise Vorticity Enhanced Mixing
}

by

\author{
David Scott Underwood
}

B.S. Aeronautics and Astronautics, Massachusetts Institute of Technology, 1993

Submitted to the Department of Aeronautics and Astronautics in partial fulfillment of the requirements for the degree of

Master of Science

at the

MASSACHUSETTS INSTITUTE OF TECHNOLOGY

September 1995

(c) Massachusetts Institute of Technology 1995. All rights reserved.

1

Author .

Department of Aeronautics and Astronautics

August 3, 1995

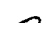

Certified by

$$
\text { August 3, } 1995
$$

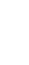

Certified by

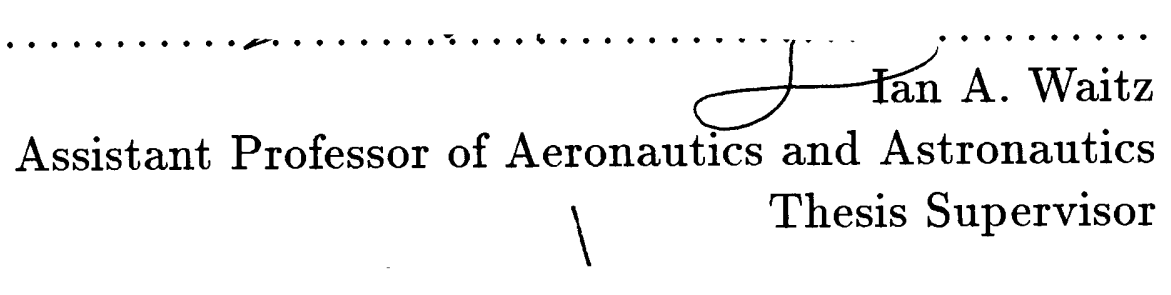

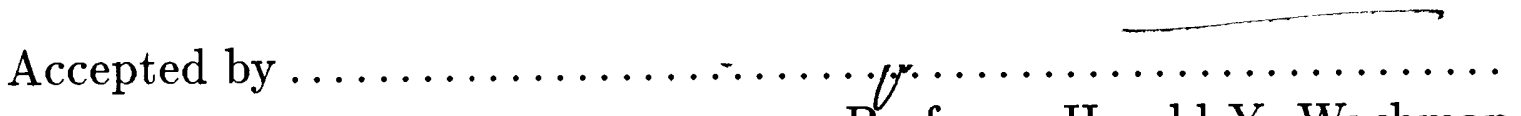

Professor Harold Y. Wachman

Chairman, Department Graduate Committee

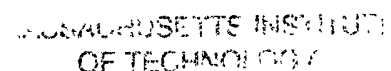

SEP 251995 



\title{
Effect of Heat Release on Streamwise Vorticity Enhanced Mixing
}

by

\section{David Scott Underwood}

\author{
Submitted to the Department of Aeronautics and Astronautics \\ on August 3, 1995, in partial fulfillment of the \\ requirements for the degree of \\ Master of Science
}

\begin{abstract}
Experiments have been carried out to determine the effect of heat release on streamwise vorticity enhanced mixing of two co-flowing streams. A lobed mixer and flat plate were examined in both reacting and non-reacting environments. Mixing rates were calculated by comparing experimental static pressure and mass-averaged total enthalpy measurements with the predictions of a quasi-one dimensional control volume model in which a mixing rate could be specified. Initial mixing rates were shown to be increased dramatically by the introduction of streamwise vorticity, while the mixing rate augmentation due to the addition of streamwise vorticity was found to be much less sensitive to the detrimental effects of heat release on mixing than the planar shear layer mixing rate. The initial mixing rates obtained for the lobed mixer were greater than those for the flat plate by a factor of between 6 and 12 for the different heat release cases. The mixing rates for the flat plate decreased by a factor of 4 for the highest heat release case compared with the zero heat release case. This decrease was in agreement with the results of previous studies. The mixing augmentation due to the streamwise vorticity decreased by a factor of 2 from zero to high heat release, while the mixing rate far downstream of the trailing edge of the lobed mixer, after the streamwise vorticity had decayed, decreased by a factor of 3.6 from zero to high heat release.
\end{abstract}

Thesis Supervisor: Ian A. Waitz

Title: Assistant Professor of Aeronautics and Astronautics 



\section{Acknowledgments}

The research presented herein could not have been carried out without the help of many individuals. Above all, I would like to thank Professor Ian Waitz for his constant good-natured guidance. Without him, this experience would have been much more painful. I also wish to thank Professor Edward Greitzer and Professor Frank Marble for their insights and encouragement, Paul Lewis for imparting his knowledge of combustion processes, and Professor James Hermanson for his valuable advice on the details of planar reacting shear layers.

Support for this work was provided by the NASA Lewis Research Center under NASA Grant NCC3-307. This support is gratefully acknowledged. I would like to thank Dr. Clarence Chang, the technical monitor for this work, Dr. Changlie Wey, Al Shott, Ghansham Dutt, Bill Kirchner, Ron Sobolewski, Edith Parrott, Harry Fuller, Kevin Roberts, and Jerry Bockmiller for their useful suggestions and long hours during my time at NASA Lewis.

I thank Dave Tew, Andy Fung, and Jan Krasnodebski for their helpful discussions on lobed mixer flows. I am also very grateful to Don Hoying and Brian Corn for making the office and the lab tolerable during frustrating times.

I am grateful to Robin Courchesne and Holly Rathbun for their help in administering the project. I also wish to thank Diana Park for providing excellent illustrations and a wealth of editorial skills and advice.

I want to thank Brett Masters for showing me that it is possible to be successful at MIT and still have a good time. I cannot thank him, Eric 'Radar' Bodnar, and Travis Atkins enough for helping me unwind every now and then. I owe you guys.

Last, but certainly not least, I wish to thank my parents Larry and Jayne Underwood for teaching me well, supporting me both financially and emotionally for so many years, and, most of all, for believing in me. Thank you so much for all your love and caring. 



\section{Contents}

1 Introduction $\quad 15$

1.1 Background and Motivation ...................... 15

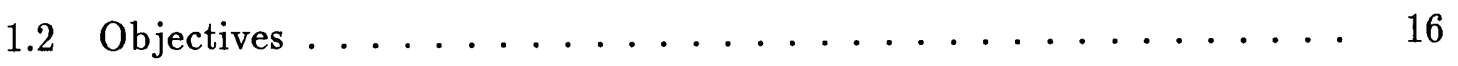

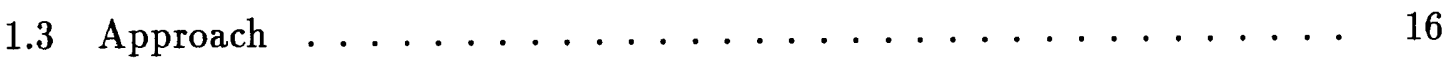

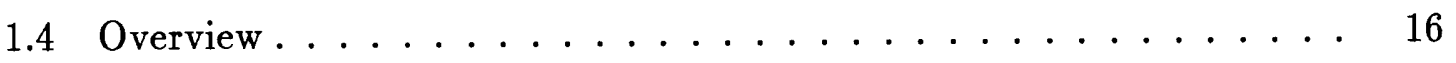

2 Experimental Apparatus 19

2.1 Reacting Shear Flow Facility . . . . . . . . . . . . 19

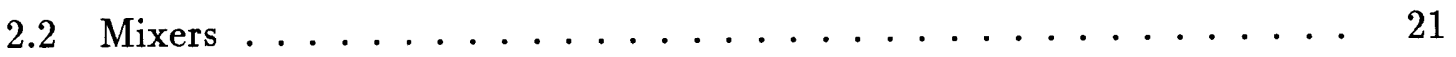

2.3 Flow Path ........................... 22

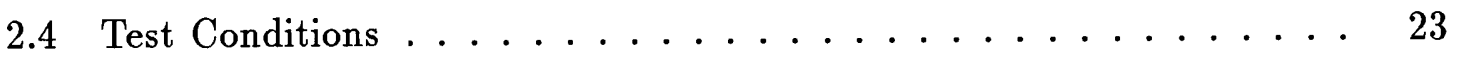

2.5 Data Acquisition . . . . . . . . . . . . . 24

2.6 Total Temperature/Total Pressure Probe . . . . . . . . . 25

3 Data Analysis $\quad 27$

3.1 Simplified Control Volume Model . . . . . . . . . . . . 27

3.2 Complete Control Volume Model . . . . . . . . . . . . 29

3.2.1 Film Cooling Mixing Rate Determination . . . . . . . . 30

4 Results 33

4.1 Total Temperature and Total Pressure Data . . . . . . . . . 33

4.1.1 Flat Plate $(\Phi=0) \ldots \ldots \ldots \ldots$

4.1.2 Flat Plate $(\Phi=1.84) \ldots \ldots \ldots \ldots$

4.1.3 Lobed Mixer $(\Phi=0) \ldots \ldots . \ldots \ldots$ 
$4.1 .4 \quad$ Lobed Mixer $(\Phi=1.84) \ldots \ldots \ldots \ldots \ldots$

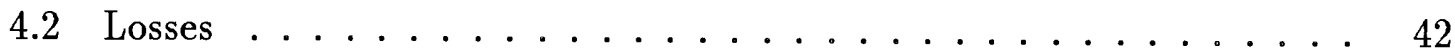

$4.3 \quad$ Static Pressure and Total Enthalpy Profiles . . . . . . . . . . . . 44

4.4 Mixing Rates and Mixedness . . . . . . . . . . . . . 47

4.5 Mixing Augmentation With Heat Release . . . . . . . . . . 51

4.6 Analytical Model . . . . . . . . . . . . . . . . . . 53

5 Conclusions $\quad 57$

$\begin{array}{ll}\text { A Thermocouple Corrections } & 61\end{array}$

A.1 Radiation Correction . . . . . . . . . . . . . . . 61

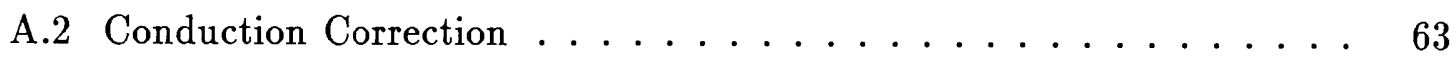

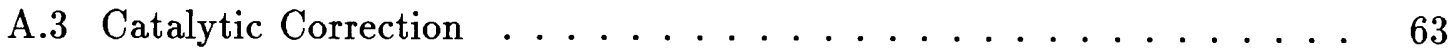

B Sensitivity Study $\quad 65$

B.1 Flat Plate . . . . . . . . . . . . . . . . 65

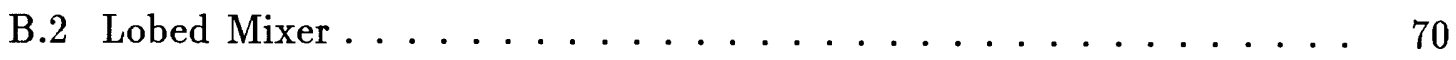

$\begin{array}{ll}\text { C Control Volume Model } & 77\end{array}$

C.1 Control Volume Equations . . . . . . . . . . . . . . 77

C.1.1 Sections 1 and $2 \ldots \ldots \ldots \ldots \ldots \ldots \ldots$

C.1.2 Section $3 \ldots \ldots \ldots \ldots \ldots \ldots$

C.2 Entrainment Model . . . . . . . . . . . . . . . . 84

C.2.1 Sections 1 and $2 \ldots \ldots \ldots \ldots \ldots \ldots \ldots$

C.2.2 Section $3 \ldots \ldots \ldots \ldots \ldots \ldots \ldots \ldots$

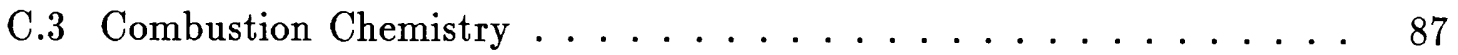




\section{List of Figures}

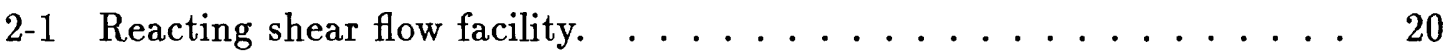

$2-2$ Test section with lobed mixer installed. . . . . . . . . . 20

2-3 Schematic of (a) flat plate and (b) lobed mixer (side and trailing edge views). . . . . . . . . . . . . . . . . . 21

2-4 Schematic of flow path in the test section. Flow is from left to right. . 22

2-5 Cross section of the flow in the test section, showing mixing and combustion zones $\ldots \ldots \ldots \ldots \ldots \ldots \ldots \ldots \ldots \ldots \ldots$

2-6 Location of total temperature and total pressure survey planes in the duct. Flow is out of the page. . . . . . . . . . . . . 24

2-7 Axial locations of survey planes. . . . . . . . . . . . . . 24

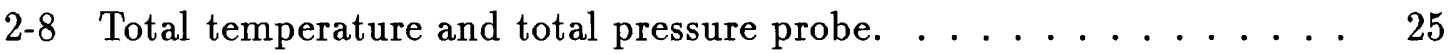

3-1 Simplified, three stream control volume model. Flow is from left to right. 28

3-2 Pressure uniformity downstream of a lobed mixer given by a numerical simulation. . . . . . . . . . . . . . . . . 29

3-3 Complete, eleven stream control volume model. . . . . . . . . . . 30

3-4 Control volume model used for film cooling mixing rate determination. 31

3-5 Experimental (o) and computational (line) static pressure profiles for film cooling mass flows, $\frac{\dot{m}_{\text {fllm }}}{\dot{m}_{\text {inlet }}}$, of (a) 0.00 , (b) 0.08 , (c) 0.17, and (d)

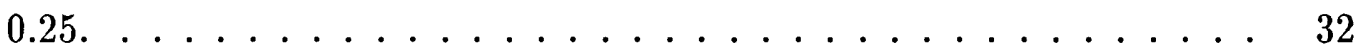

4-1 Total temperature surveys for the flat plate with zero heat release $(\Phi=0) .34$

4-2 Total pressure surveys for the flat plate with zero heat release $(\Phi=0) . \quad 35$ 
4-3 Total temperature surveys for the flat plate with high heat release $(\Phi=1.84) \ldots \ldots \ldots \ldots \ldots$

4-4 Total pressure surveys for the flat plate with high heat release $(\Phi=1.84) .37$

4-5 Total temperature surveys for the lobed mixer with zero heat release $(\Phi=0) \ldots \ldots \ldots \ldots \ldots \ldots$

4-6 Total pressure surveys for the lobed mixer with zero heat release $(\Phi=0) .39$

4-7 Total temperature surveys for the lobed mixer with high heat release $(\Phi=1.84) . \ldots \ldots \ldots 40 \ldots \ldots$

4-8 Total pressure surveys for the lobed mixer with high heat release $(\Phi=1.84) .41$

4-9 Experimental (o) and computational (line) loss coefficients for (a) flat plate and (b) lobed mixer. . . . . . . . . . . . . . . 43

4-10 Flat plate experimental (o) and computed (line) static pressure and total enthalpy profiles for (a) $\Phi=0$, (b) $\Phi=1.48$, and (c) $\Phi=1.84 \ldots \ldots$

4-11 Lobed mixer experimental ( $\circ$ ) and computed (line) static pressure and total enthalpy profiles for (a) $\Phi=0$, (b) $\Phi=1.48$, and (c) $\Phi=1.84$. . . . 46

4-12 Mixedness curves with error bands for (a) flat plate and (b) lobed mixer. 49

B-1 Maximum (solid) and minimum (dashed) static pressure profiles for the flat plate with zero heat release $(\Phi=0) \ldots \ldots$. . . . . . . . 66

B-2 Maximum and minimum mixing rates (dashed) and best mixing rate (solid) for the flat plate with zero heat release $(\Phi=0) \ldots \ldots 66$

B-3 Maximum (solid) and minimum (dashed) static pressure profiles for the flat plate with low heat release $(\Phi=1.48) . \ldots \ldots 7$

B-4 Maximum (solid) and minimum (dashed) mass averaged total enthalpy profiles for the flat plate with low heat release $(\Phi=1.48)$. . . . . . . 68

B-5 Maximum and minimum mixing rates associated with the static pressure profiles (dashed) and the total enthalpy profiles (dotted) along with the best mixing rate (solid) for the flat plate with low heat release $(\Phi=1.48) \ldots \ldots \ldots \ldots \ldots \ldots \ldots$ 
B-6 Maximum (solid) and minimum (dashed) static pressure profiles for the flat plate with high heat release $(\Phi=1.84) \ldots \ldots \ldots$

B-7 Maximum (solid) and minimum (dashed) mass averaged total enthalpy profiles for the flat plate with high heat release $(\Phi=1.84) \ldots . .$. .

B-8 Maximum and minimum mixing rates associated with the static pressure profiles (dashed) and the total enthalpy profiles (dotted) along with the best mixing rate (solid) for the flat plate with high heat release $(\Phi=1.84) \ldots \ldots \ldots \ldots \ldots \ldots$

B-9 Maximum (solid) and minimum (dashed) static pressure profiles for the lobed mixer with zero heat release $(\Phi=0) . \ldots \ldots$

B-10 Maximum and minimum mixing rates (dashed) and best mixing rate (solid) for the lobed mixer with zero heat release $(\Phi=0) . \ldots .$.

B-11 Maximum (solid) and minimum (dashed) static pressure profiles for the lobed mixer with low heat release $(\Phi=1.48) \ldots \ldots \ldots$

B-12 Maximum (solid) and minimum (dashed) mass averaged total enthalpy profiles for the lobed mixer with low heat release $(\Phi=1.48) \ldots . .$.

B-13 Maximum and minimum mixing rates associated with the static pressure profiles (dashed) and the total enthalpy profiles (dotted) along with the best mixing rate (solid) for the lobed mixer with low heat release $(\Phi=1.48) \ldots \ldots \ldots \ldots$

B-14 Maximum (solid) and minimum (dashed) static pressure profiles for the lobed mixer with high heat release $(\Phi=1.84) . \ldots \ldots$

B-15 Maximum (solid) and minimum (dashed) mass averaged total enthalpy profiles for the lobed mixer with high heat release $(\Phi=1.84)$. . . .

B-16 Maximum and minimum mixing rates associated with the static pressure profiles (dashed) and the total enthalpy profiles (dotted) along with the best mixing rate (solid) for the lobed mixer with high heat release $(\Phi=1.84) \ldots \ldots \ldots \ldots \ldots \ldots$ 


\section{List of Tables}

4.1 Experimental and computational loss coefficients, L, for the lobed mixer and flat plate. . . . . . . . . . . . . . . 42

4.2 Flat plate and lobed mixer mixing rates. . . . . . . . . 52

4.3 Observed mixing augmentation rates, $d M_{a} \ldots \ldots \ldots$

4.4 Comparison of flat plate mixing rates for varying heat release with the results of Hermanson and Dimotakis. . . . . . . . . . . . 53

4.5 Comparison of expected and observed values of $d M_{a} \ldots \ldots \ldots$ 


\section{Chapter 1}

\section{Introduction}

\subsection{Background and Motivation}

Lobed mixers are often employed to provide rapid, low loss mixing between two coflowing fluid streams. This rapid mixing has been identified as having applications in low $N O_{x}$ combustors such as those being developed for use on the High Speed Civil Transport, a proposed supersonic transport aircraft.[1] These applications include rapid mixing of the primary combustion stream with air in the quenching section of a rich-quench-lean combustor, and generation of high strain rates in the flow downstream of a fuel injection point. High strain rates, if on the order of the chemical kinetic rates, may affect $N O_{x}$ formation and yield lower pollutant concentrations.

The increased mixing associated with lobed mixers stems from two sources; the increased interfacial area between the two streams due to the increased trailing edge length of a lobed mixer when compared to a flat plate, and the cross-stream convection associated with the streamwise vorticity generated by the lobed mixer.[2][3] Each of these sources for enhanced mixing also generate total pressure losses.[4] The loss associated with the increased trailing edge length is due to the increased surface area of the lobed mixer relative to the flat plate. The loss associated with the generation of streamwise vorticity is due to the diffusion of the kinetic energy associated with the streamwise component of the shed vorticity into heat.

Most of the research on mixing enhancement using lobed mixers has focused on 
non-reacting flows.[2][5][6][7] A small amount of research has been done on reacting lobed mixer flows, and the potential for enhanced mixing in reacting flows has been demonstrated.[8] In particular, the flow field downstream of a lobed mixer in a reacting environment has been visualized. However, there has been no quantification of the effect of heat release on streamwise vorticity enhanced mixing.

\subsection{Objectives}

The present research was aimed at providing an understanding of the effect of heat release on streamwise vorticity enhanced mixing. The specific research objectives were:

1. To quantify the effect of significant heat release on the mixing rate augmentation associated with the introduction of streamwise vorticity downstream of a lobed mixer.

2. To add to the experimental validation of lobed mixer results in cold flow as well as flat plate results in both reacting and non-reacting environments.

\subsection{Approach}

Experiments were conducted at the NASA Lewis Research Center in a reacting shear flow facility. A flat plate and a lobed mixer were tested in reacting and non-reacting flows. Data included total temperature and total pressure surveys as well as wall static pressures. The data were used to determine the mixing rate via comparison of static pressure and mass flux weighted total enthalpy profiles obtained from the experiment with one dimensional control volume results.

\subsection{Overview}

The thesis begins with a description of the experimental apparatus, test conditions, and data acquisition procedures in Chapter 2. The control volume models used to de- 
termine mixing rates are then presented in Chapter 3 . In Chapter 4 , the experimental results are presented and discussed. Chapter 5 contains a summary and conclusions. 


\section{Chapter 2}

\section{Experimental Apparatus}

\subsection{Reacting Shear Flow Facility}

The experiments were conducted in the NASA Lewis reacting shear flow facility. The test section was 8 inches wide, 4 inches high, and 25 inches long and exhausted to atmospheric pressure. Visual access to the flow field was through two quartz windows. The upper and lower walls of the test section were hinged to adjust the axial pressure gradient. However, the maximum divergence angles were too small to maintain a zero axial pressure gradient during the runs, so the walls were set at a $1^{\circ}$ divergence angle throughout the tests. A photograph of the facility is shown in Figure 2-1. The flow is from left to right. Figure $2-2$ is a photograph of the test section with the lobed mixer installed.

The facility was capable of delivering air via a non-vitiating preheater at $820 \mathrm{~K}$ or less with a Mach number of 1 or less. Fuel, composed of hydrogen and nitrogen, could be delivered between $340 \mathrm{~K}$ and $380 \mathrm{~K}$ with a Mach number of 0.5 or less. These conditions were not sufficient to maintain flame stability at the trailing edge of the mixer. Therefore, an additional preheater was used which provided an extra $100 \mathrm{~K}$ of temperature rise in the air stream. This additional temperature rise was enough to ensure flame stability. This second preheater was vitiating, utilizing hydrogen as the fuel. It was positioned across the air duct approximately 3 meters upstream of the trailing edge. This position was upstream of all flow conditioning screens and 


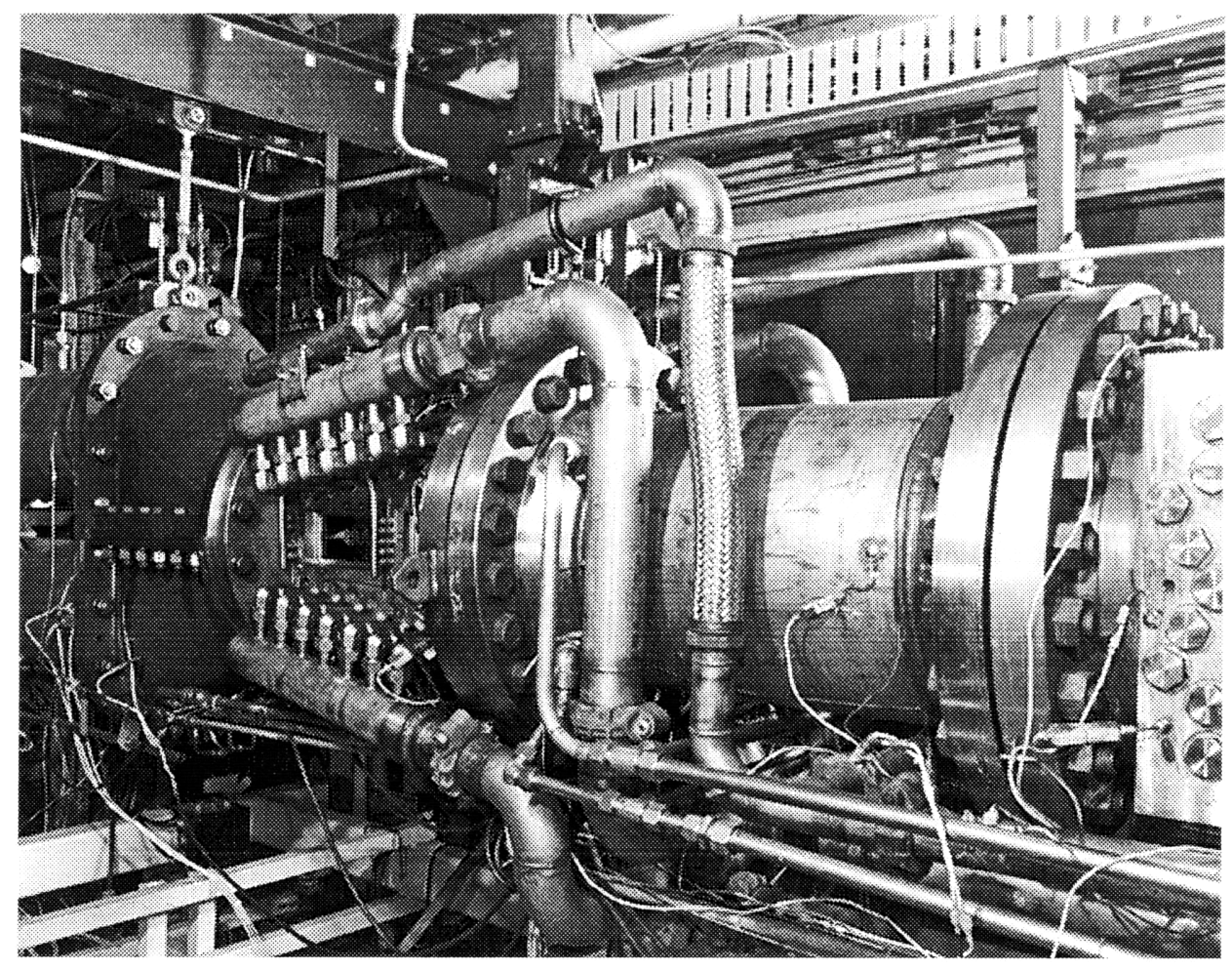

Figure 2-1: Reacting shear flow facility.

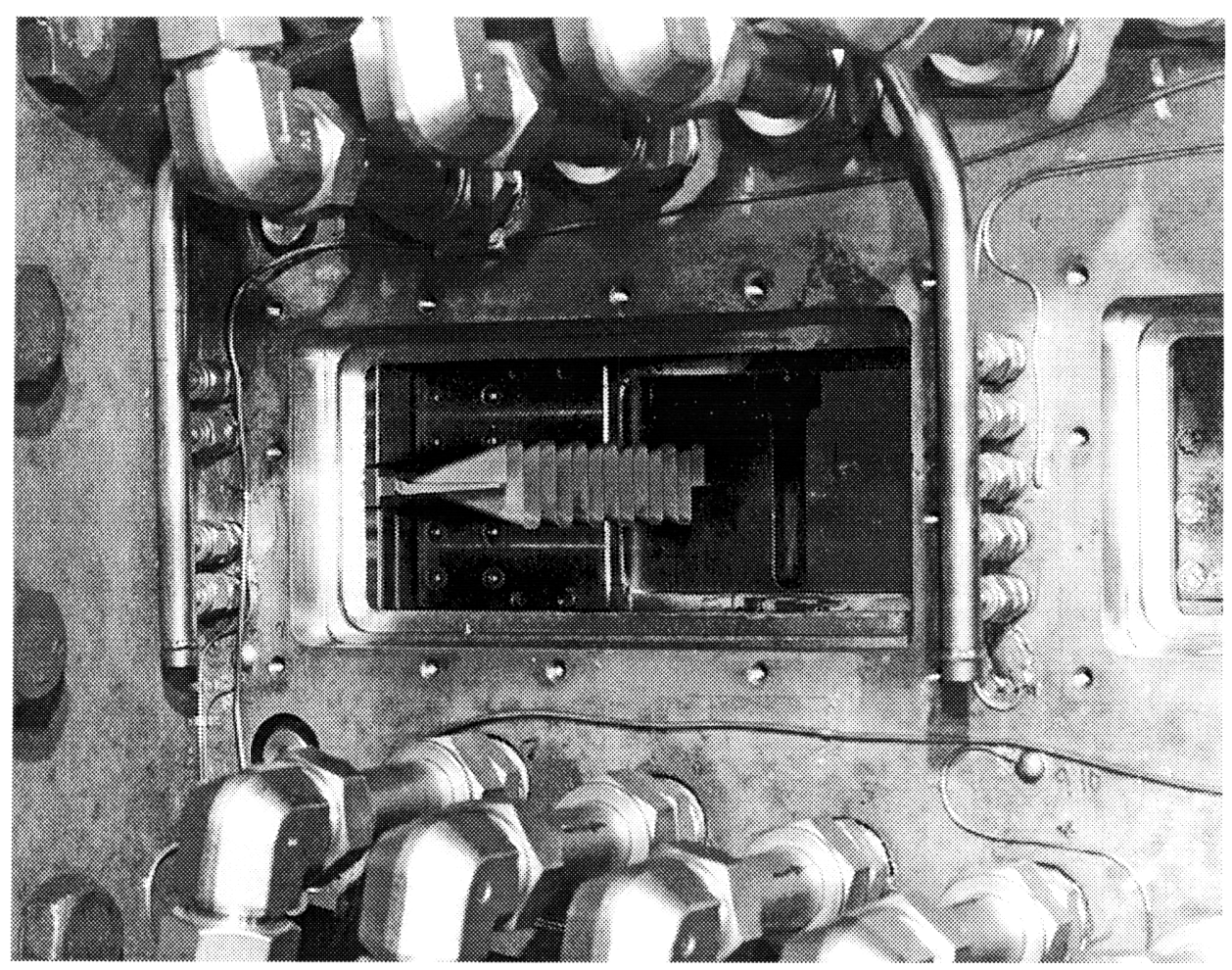

Figure 2-2: Test section with lobed mixer installed. 


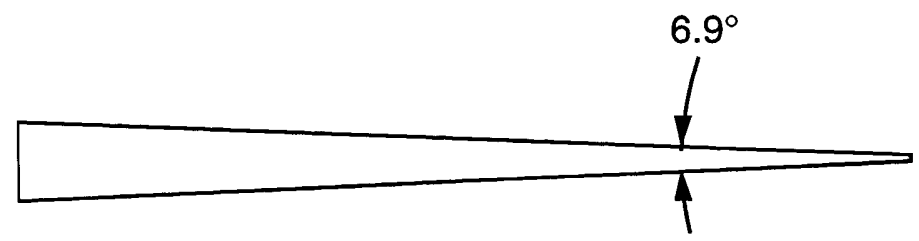

(a)
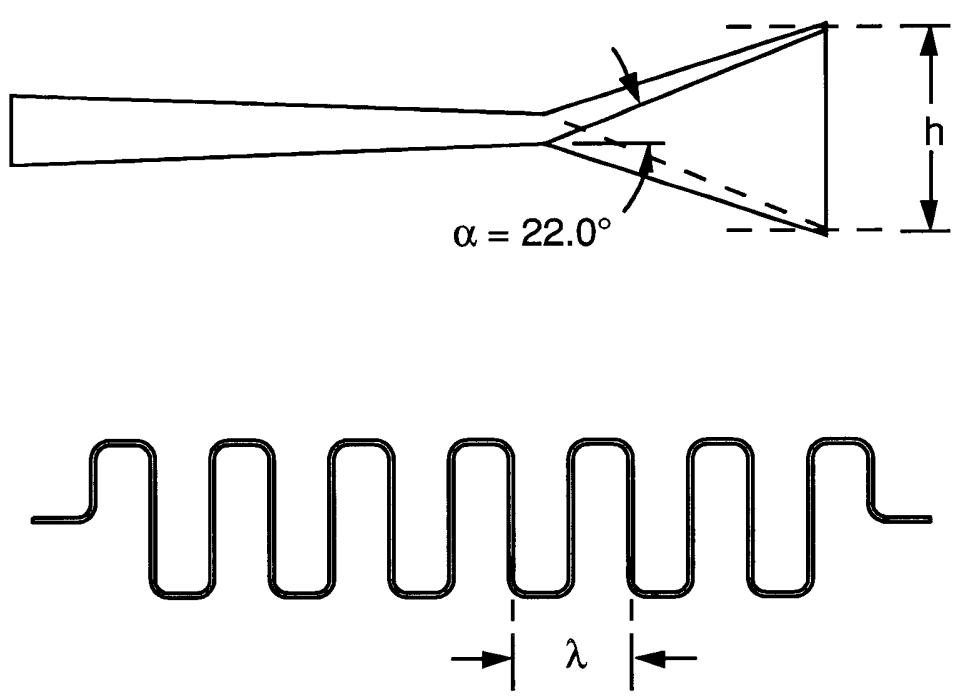

(b)

Figure 2-3: Schematic of (a) flat plate and (b) lobed mixer (side and trailing edge views).

honeycomb yielding a temperature non-uniformity at the trailing edge of less than $10 \mathrm{~K}$.

\subsection{Mixers}

Schematics of the two geometries tested are shown in Figure 2-3. The first was a flat plate with a convergence angle of $6.9^{\circ}$ and trailing edge thickness of 0.040 inches. The second was a lobed mixer with a penetration angle, $\alpha$, of $22^{\circ}$, lobe wavelength, $\lambda$, of 1 inch, height to wavelength ratio, $\mathrm{h} / \lambda$, of 1.25 , and trailing edge thickness of 0.040 inches. This geometry is typical of current lobed mixer technology and produced a non-dimensional circulation, $\Gamma / \bar{U} \lambda$, of 1 . 


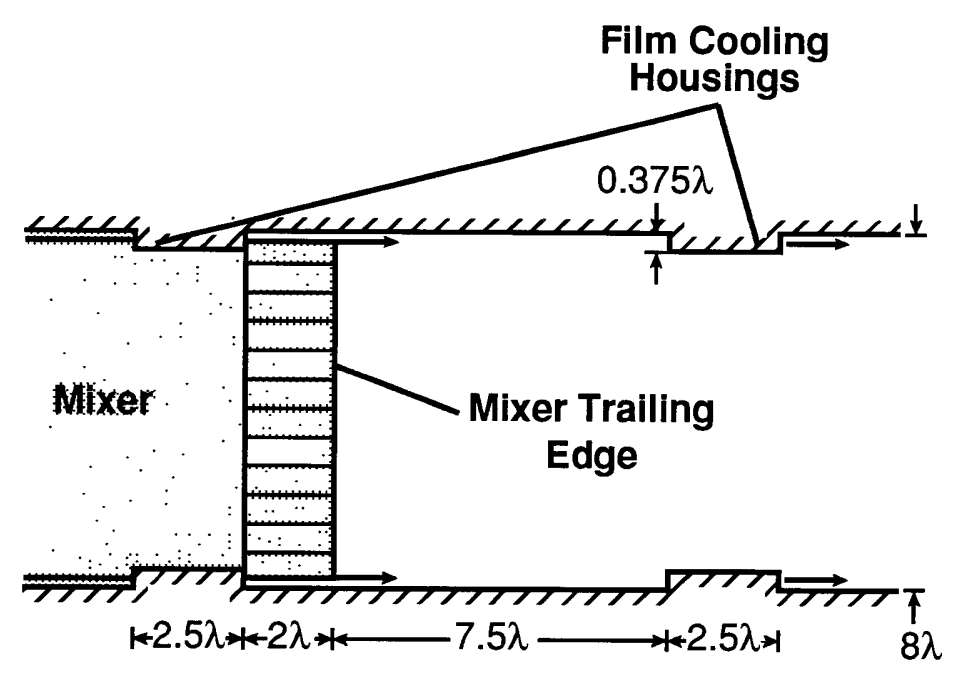

Figure 2-4: Schematic of flow path in the test section. Flow is from left to right.

\subsection{Flow Path}

Figure 2-4 shows the test section flow path with flow from left to right. The protrusions into the test section just upstream of the trailing edge of the mixer and then again at 7.5 lobe wavelengths downstream are housings for film cooling injection. Immediately downstream of the housings were the windows for which the cooling flow was provided. The film cooling gas was air.

The result of the cooling air injection is shown schematically in Figure 2-5. The main mixing zone with combustion between the main fuel and air streams is shown as the hashed, horizontal region in the center of the duct. There were also two mixing zones created between the film cooling air and each of the main streams, shown as the hashed vertical regions on the sides of the duct. The portion of the mixing layer between the film cooling air and the main fuel and combustion zones yielded additional heat release beyond that associated with the primary mixing/reaction zone. The effect of the additional mass injection and heat release due to the film cooling was accounted for when reducing the data and will be discussed in detail in Section 3.2. 


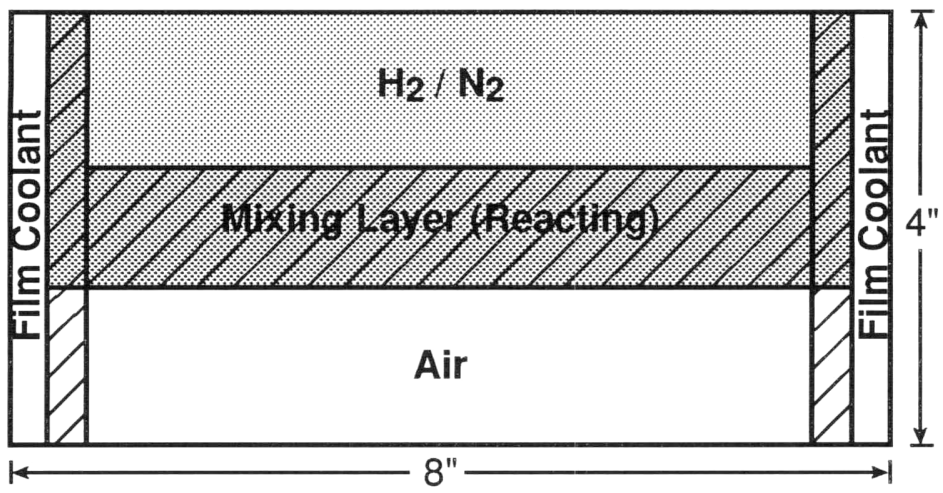

Figure 2-5: Cross section of the flow in the test section, showing mixing and combustion zones

\subsection{Test Conditions}

The flat plate and lobed mixer were tested in both reacting and non-reacting flows. The non-reacting flow conditions consisted of nitrogen at $360 \mathrm{~K}$ in the low speed stream and air at $820 \mathrm{~K}$ in the high speed stream. The inlet velocities of the low and high speed streams were 85 and $170 \mathrm{~m} / \mathrm{s}$, respectively, yielding a velocity ratio, $\mathrm{r}=U_{2} / U_{1}$, between the two streams of 0.5 , where $U_{2}$ is defined as the low speed flow velocity and $U_{1}$ as the high speed flow velocity.

Two reacting flow conditions were tested, corresponding to two different concentrations of hydrogen in the low speed stream. Defining an equivalence ratio, $\Phi$, as the ratio of the mass flow of hydrogen to oxygen at the inlet divided by this ratio at stoichiometric conditions, tests were run with $\Phi=1.48$ in the low heat release case and $\Phi=1.84$ in the high heat release case. During reacting runs, the fuel stream was heated to $340 \mathrm{~K}$ and the air stream was heated to $920 \mathrm{~K}$. The nominal velocity ratio between the two streams was 0.5 with the inlet velocities being the same as for the non-reacting runs. For complete mixing, the total enthalpy rise, $\Delta h_{t} / h_{t, \text { inlet }}$, for the high heat release case $(\Phi=1.84)$ was approximately $300 \%$, in the range of practical interest for a gas turbine combustor. The total enthalpy rise for the low heat release case $(\Phi=1.48)$ was approximately $200 \%$. 


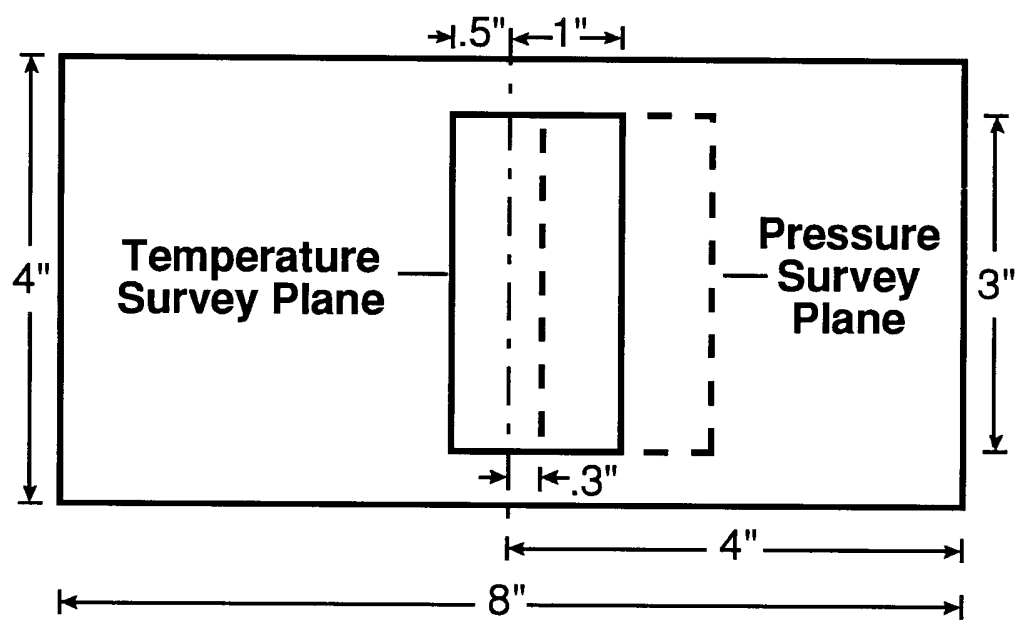

Figure 2-6: Location of total temperature and total pressure survey planes in the duct. Flow is out of the page.

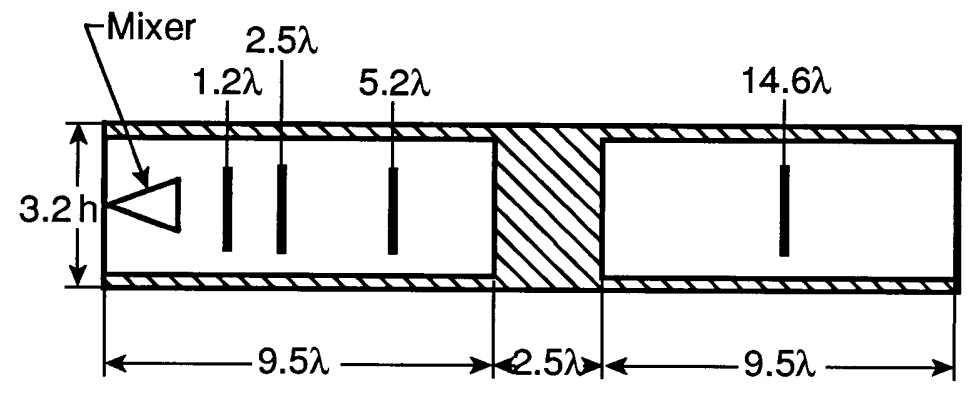

Figure 2-7: Axial locations of survey planes.

\subsection{Data Acquisition}

Planar total pressure and total temperature surveys were taken for each geometry at each of the three flow conditions described in Section 2.4. These surveys were taken at distances of $1.2,2.5,5.2$, and 14.6 lobe wavelengths downstream of the trailing edge of the mixer. The flow was sampled every 0.10 lobe wavelengths in a plane 3 lobe wavelengths high and 1.5 lobe wavelengths wide. Figure 2-6 shows a survey plane and its relation to the various regions of the flow field shown in Figure 2-5, while Figure 27 shows the position of the four survey planes in relation to the trailing edge. The uncertainty in the total temperature measurements, after radiation, conduction, and catalytic corrections were made was $10 \mathrm{~K}$, while the uncertainty in the total pressure measurements was 0.04 psi.

Static pressure data was taken at five points downstream of the trailing edge. 


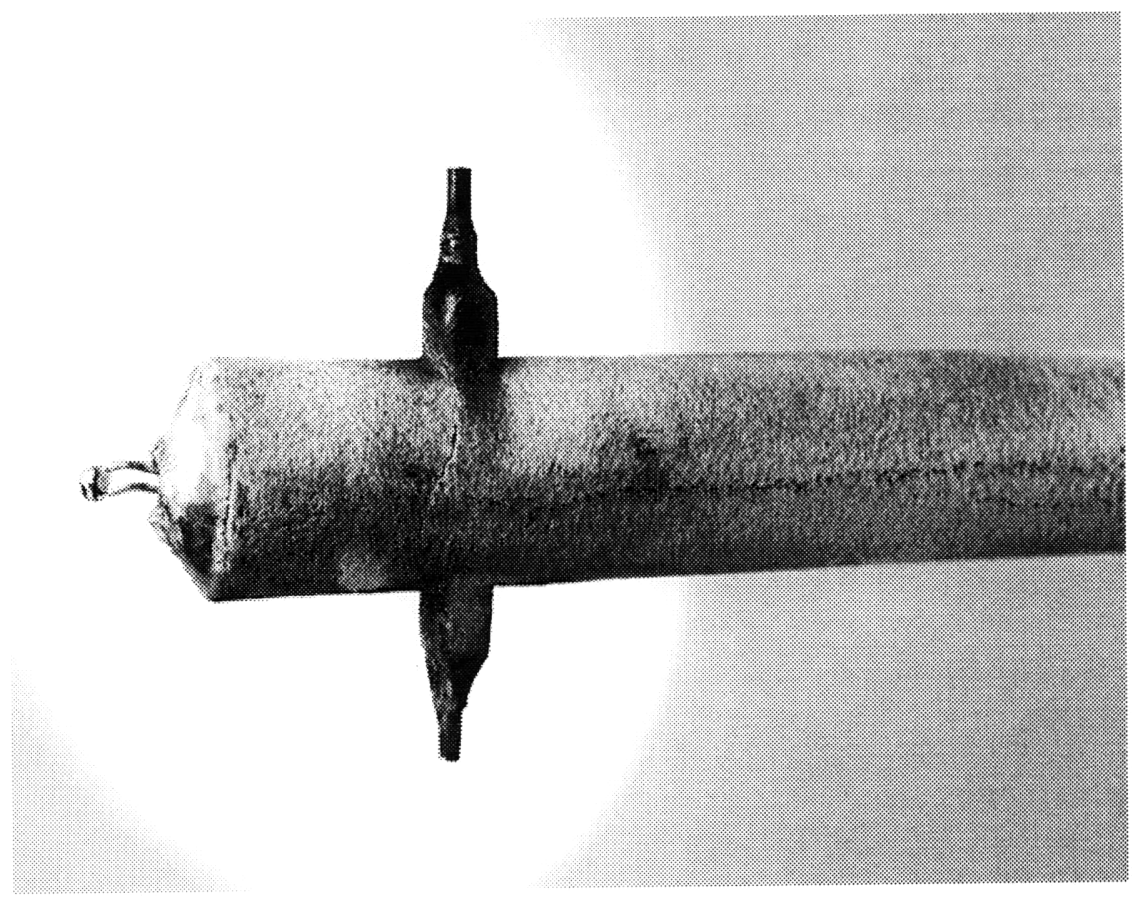

Figure 2-8: Total temperature and total pressure probe.

Four of these points corresponded to the four survey planes mentioned above. The fifth static tap was located at 4.0 lobe wavelengths downstream of the trailing edge. The uncertainty in the static pressure measurements was 0.02 psi for all tests.

\subsection{Total Temperature/Total Pressure Probe}

Due to minimal scheduled test time, total temperature and total pressure measurements were incorporated into a single probe. This probe was water cooled and coated with a ceramic thermal barrier made of a yittrium stabilized zirconia oxide typical of modern turbine blade thermal barriers. The diameter of the probe body was 0.5 inches to accommodate the water cooling passages. Figure 2-8 shows the end of the probe. The tubes on either side are the total pressure probes. They were made of a molybdenum-rhenium alloy with a inner diameter of 0.040 inches, outer diameter of 0.060 inches, and length of 0.375 inches. There was one probe on each side since the probe had to be turned over in order to survey the plane at $\mathrm{x} / \lambda=1.2$. Appendix $\mathrm{A}$ gives more details about the thermocouple. 


\section{Chapter 3}

\section{Data Analysis}

The main objective of the experiments was to determine the mixing rate in the primary mixing layer shown in Figure 2-5. However, the film coolant flows complicated this by producing additional reacting and non-reacting mixing zones. A quasi-one dimensional control volume model was developed as a tool for determining the primary mixing rate at each test condition. The control volume model allowed the specification of a single mixing rate between the two primary streams, while taking the film cooling zones into account. The primary stream mixing rate was iterated on until the static pressure and mass-averaged total enthalpy profiles given by the control volume model agreed with those measured in the experiment. Before describing the complete model in Section 3.2, a simplified model will be discussed in Section 3.1 to illustrate the basic data analysis technique.

\subsection{Simplified Control Volume Model}

The primary mixing zone was modeled as two streams with a growing shear layer between them, as depicted in Figure 3-1. The mixing rate was specified by setting the amount of fluid entrained into the shear layer from the outer two streams. The mixing rate could be varied until the static pressure and total enthalpy profiles agreed with those obtained from the experiment.

The analysis employed the simplified reaction mechanism 


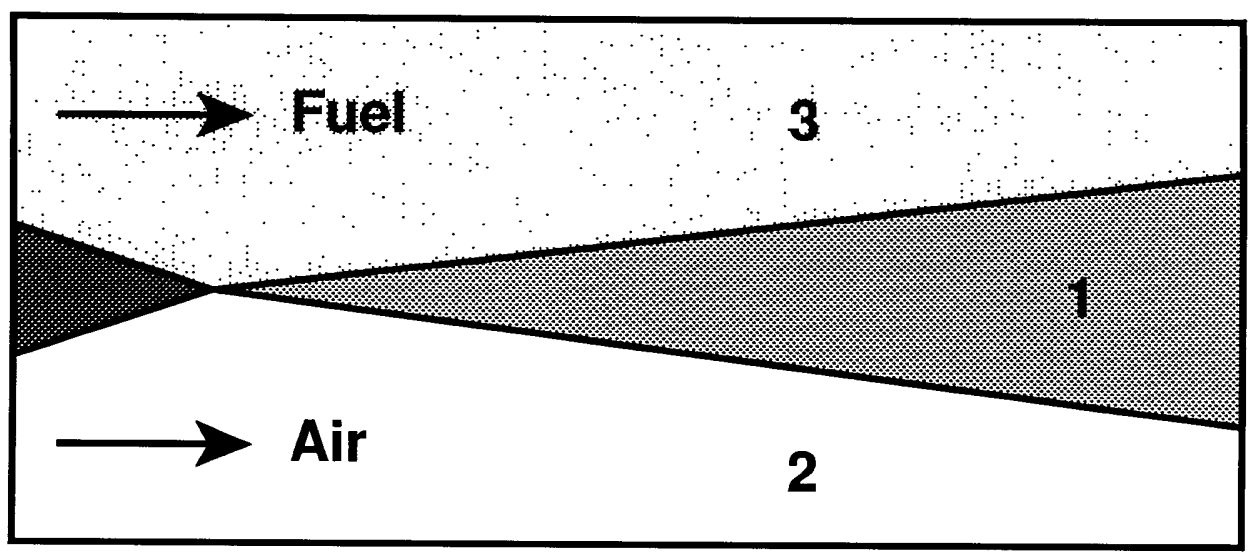

Figure 3-1: Simplified, three stream control volume model. Flow is from left to right.

$$
\left(H_{2}+\frac{1}{\alpha} N_{2}\right)+\frac{1}{2 \psi}\left(O_{2}+3.76 N_{2}\right) \rightarrow H_{2} O+\left(\frac{1}{2 \psi}-\frac{1}{2}\right) O_{2}+\left(\frac{1.88}{\psi}+\frac{1}{\alpha}\right) N_{2}
$$

where $\alpha$ is the ratio of moles of hydrogen to moles of nitrogen, and $\psi$ is the fuel to air ratio, $\Phi$, defined before, multiplied by the ratio of the number of moles of hydrogen to oxygen being entrained into the shear layer at every downstream location. It was assumed that all reactants entrained into the shear layer combusted completely and instantaneously. It was also assumed that the pressure was uniform in any given plane downstream of the trailing edge.

The assumption of uniform static pressure was evaluated using a three-dimensional Navier-Stokes code.[9] A numerical simulation of the lobed mixer flow was performed for non-reacting flow with a velocity ratio, $\mathrm{r}$, of 0.5 , penetration angle, $\alpha$, of $22^{\circ}$, and height to wavelength ratio, $h / \lambda$, of 1.0. The results of this simulation are shown in Figure 3-2. The lowest and highest static pressure coefficients, $C_{p}=\Delta p / \bar{q}$, (where $\bar{q}$ is the average inlet dynamic head, $\left.\bar{\rho} \bar{u}^{2} / 2\right)$ in a plane were compared to the massaveraged $C_{p}$ and found to deviate by less than \pm 0.1 near the trailing edge. After 4 wavelengths downstream, the deviation was less than \pm 0.01 . Thus the static pressure non-uniformities were small in the near field of the mixer and negligible in the far field, and therefore, the assumption of uniform static pressure was justified. 


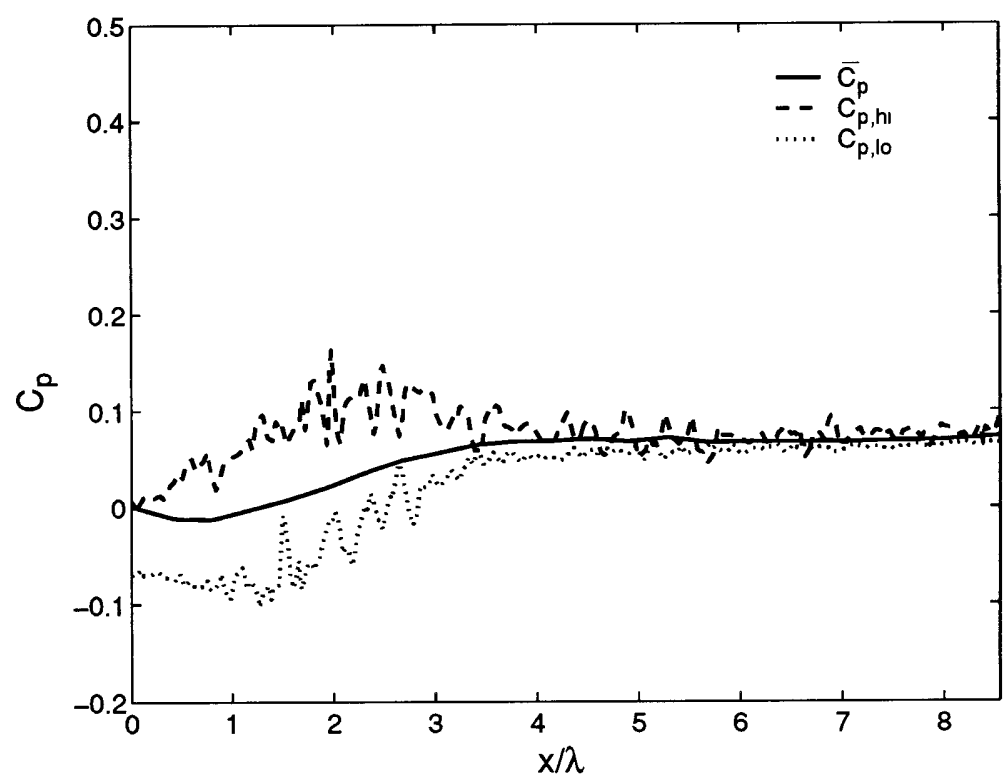

Figure 3-2: Pressure uniformity downstream of a lobed mixer given by a numerical simulation.

\subsection{Complete Control Volume Model}

As noted in Section 2.3, there was mixing between the main streams and the film cooling air as well as combustion due to part of this mixing. There were also changes in duct area due to the film cooling housings and wall divergence. A more complete control volume model, shown in Figure 3-3, was developed to account for these effects. The mathematical details of this model are presented in Appendix C.

The main inflow streams and shear layer were denoted by streams 1,2 , and 3 . These three streams composed the simplified model discussed in Section 3.1. Two film cooling streams were injected on the top and bottom, shown as streams 6 and 7. These streams entered with different velocities than the main streams. The shear layer between the fuel stream and the top film cooling stream, stream 5, combusted, while the bottom shear layer, stream 4 , did not. At $x / \lambda=7.5$, a step change in the duct area occurred where the second film cooling housing began. Another 2.5 wavelengths downstream of this, the second mass injection occurred. There was reaction in the shear layer labeled stream 11, while no reaction occurred in the bottom shear layer, stream 10 . 


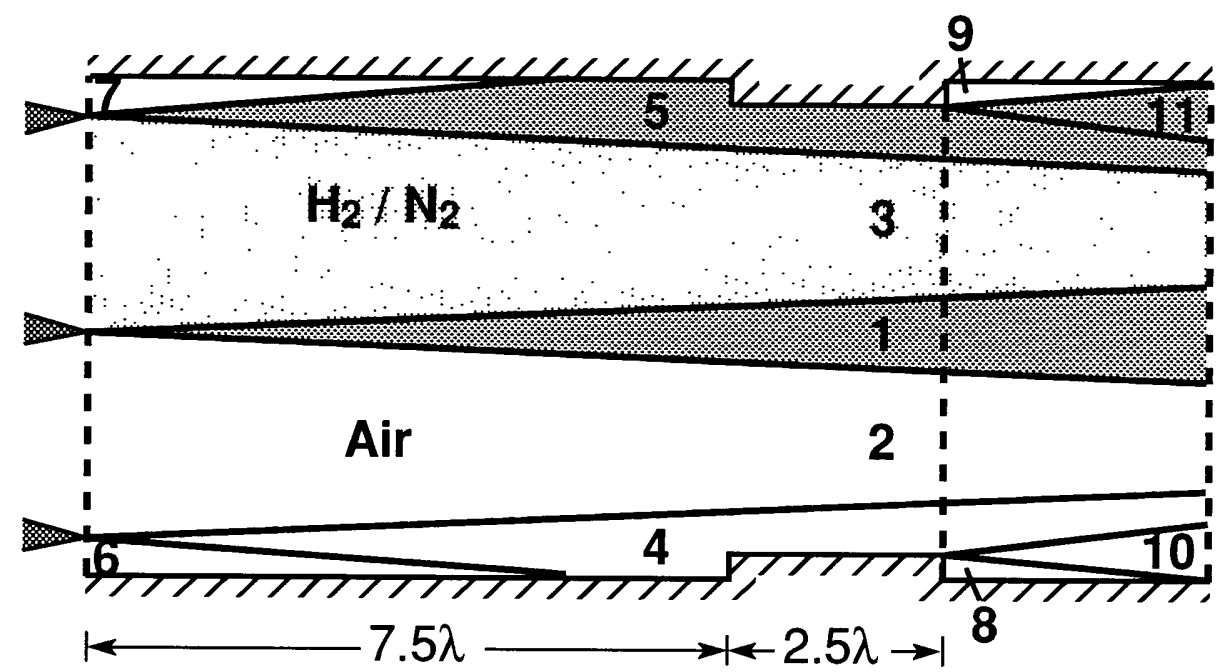

Figure 3-3: Complete, eleven stream control volume model.

The mixing rates for the film cooling shear layers were specified based on results from experimental calibration tests as described below in Section 3.2.1. Then the main mixing rate for stream 1 was changed until the predicted static pressure and mass-averaged total enthalpy profiles matched the experimental static pressure and mass-averaged total enthalpy profiles. The criteria adopted for determining the best match was that the computed static pressure be within $\pm 5 \%$ of the experimental value for no less than four of the five measurement points. The criteria adopted for matching the total enthalpy was that the computed total enthalpy be within $\pm 5 \%$ of the experimental value for no less than three of the four measurement points. These criteria were used throughout for all static pressure and total enthalpy matching.

\subsubsection{Film Cooling Mixing Rate Determination}

Film cooling mixing rates were determined by using the simplified control volume model with no reaction to model the mixing of one film cooling layer. Figure 34 shows the model used. The static pressure profile given by the simplified model was compared to experimental calibration data with varying film cooling flow rates. Effects due to mixing of the two primary inflow streams were minimized since these tests were run with a flat plate at a velocity ratio of 1 and zero heat release. At these conditions, pressure changes were due solely to changes in duct area and mixing 


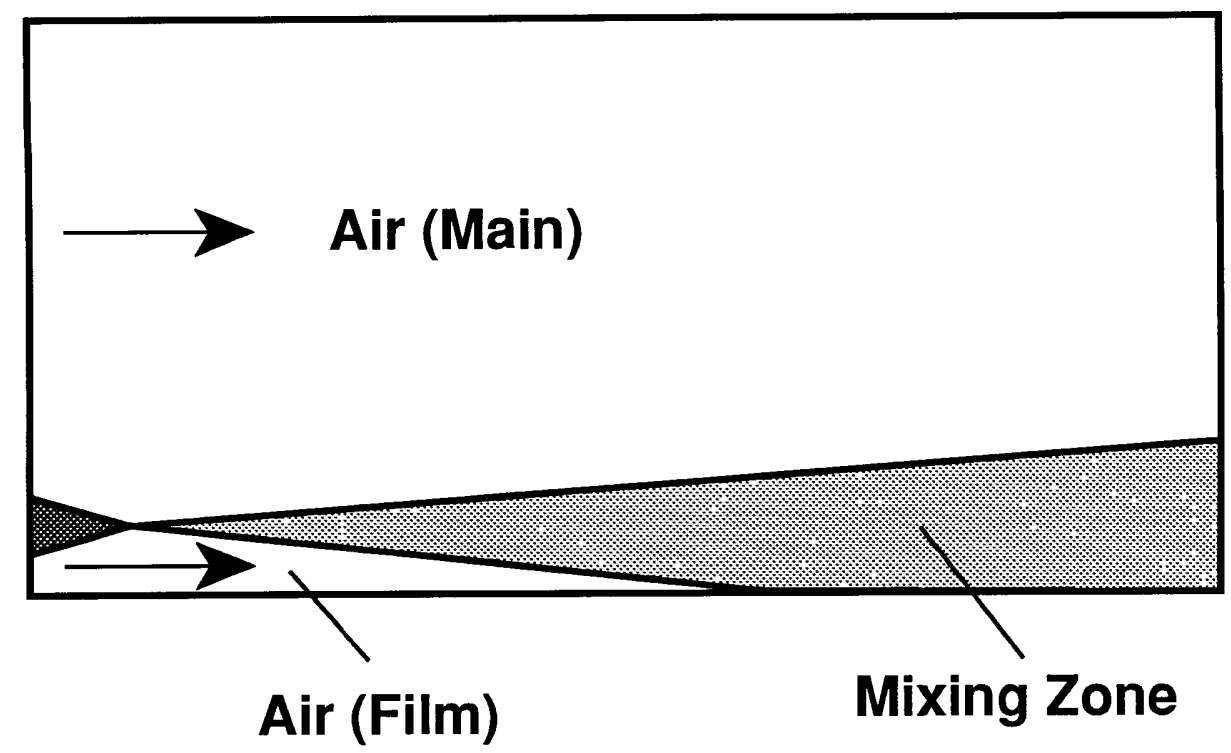

Figure 3-4: Control volume model used for film cooling mixing rate determination.

between the film cooling air and the main air streams.

A single film cooling mixing rate was specified so that the experimental and computed static pressure profiles agreed. This mixing rate was used for the two nonreacting film cooling shear layers, streams 4 and 10 as shown in Figure 3-3. Figure 3-5 shows the best match between static pressure profiles for the four film cooling flow rates tested.

The calibration tests could only be carried out for non-reacting conditions. The mixing rates for the two reacting film cooling shear layers were determined by scaling the cold flow mixing rates using the empirically derived scaling law of Hermanson and Dimotakis for the entrainment of fluid into a reacting shear layer.[10] Hermanson and Dimotakis have shown that the entrainment of fluid into a shear layer decreases with increasing heat release. Their data for a flat plate with varying heat release was used to determine the relative reduction in mixing rate at the heat releases encountered in the current experiments. This mixing rate was then used for the two reacting film cooling shear layers, streams 5 and 11 in Figure 3-3. The mixing rates for streams 4, 5,10 , and 11 were left constant at these computed values during the final iteration on the main mixing rate in stream 1.

Comparison of the static pressure changes associated with the film cooling flow, 
shown in Figure 3-5, with those associated with the main mixing process (see Figures 4-10 and 4-11) shows that the relative effect of the film cooling was small compared to the trends measured. Therefore, the relative magnitude of the film cooling correction was small.

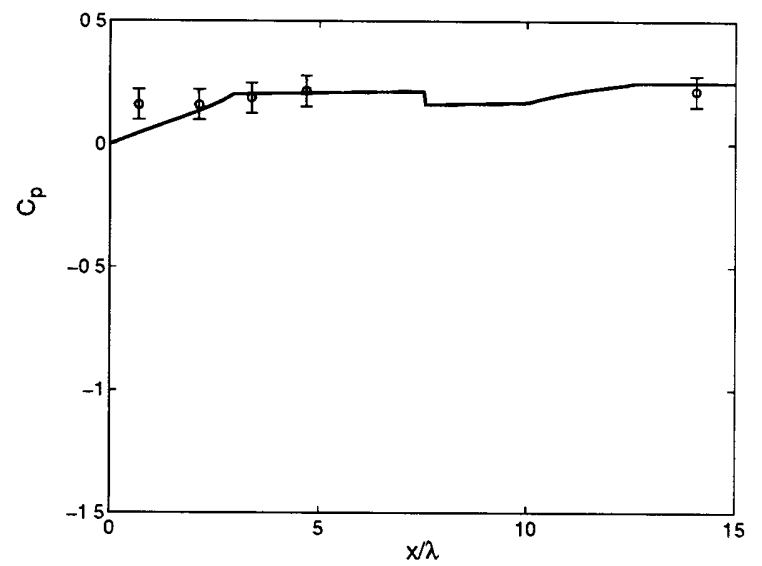

(a) $\frac{\dot{m}_{f i l m}}{\dot{m}_{\text {inlet }}}=0.00$

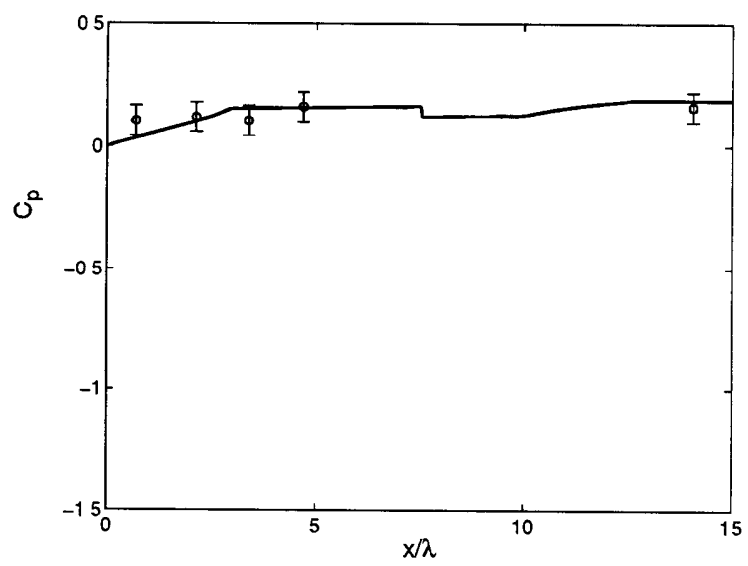

(c) $\frac{\dot{m}_{\text {film }}}{\dot{m}_{\text {inlet }}}=0.17$

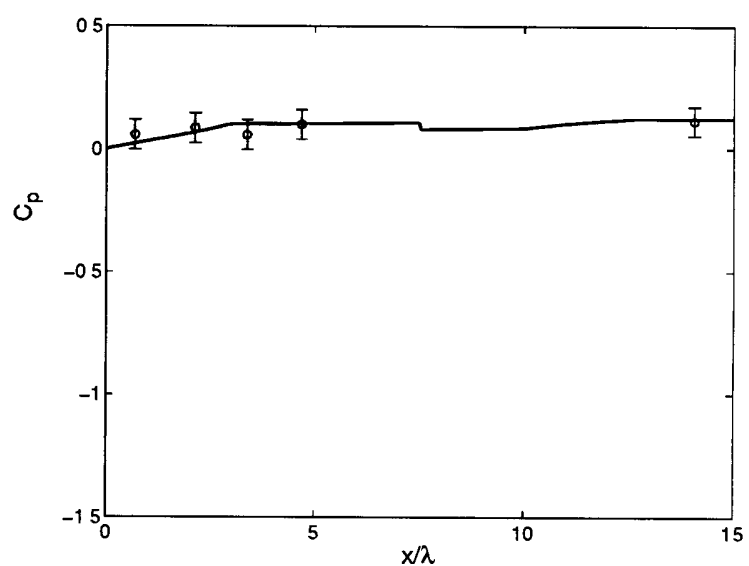

(b) $\frac{\dot{m}_{\text {film }}}{\dot{m}_{\text {inlet }}}=0.08$

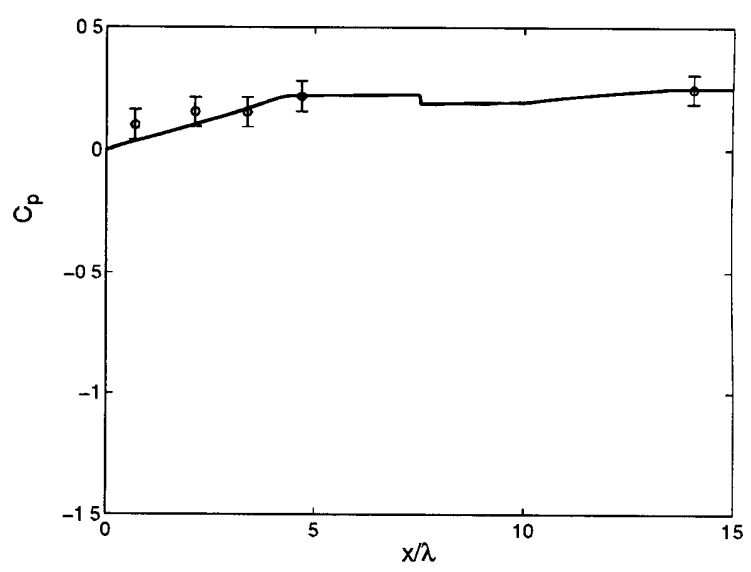

(d) $\frac{\dot{m}_{\text {film }}}{\dot{m}_{\text {inlet }}}=0.25$

Figure 3-5: Experimental (o) and computational (line) static pressure profiles for film cooling mass flows, $\frac{\dot{m}_{\text {film }}}{\dot{m}_{\text {inlet }}}$, of (a) 0.00, (b) 0.08, (c) 0.17 , and (d) 0.25 . 


\section{Chapter 4}

\section{Results}

Total temperature and total pressure surveys are presented in Section 4.1 followed by a comparison of the measured losses with the results of computations in Section 4.2. Mass-averaged total enthalpy and static pressure data are presented in Section 4.3, and in Section 4.4 the mixing rates derived from the experimental data using the control volume model are presented and discussed. Section 4.5 presents the results for the augmented mixing rates with heat release, and finally, an analytical model for the effect of heat release on streamwise vorticity enhanced mixing is presented in Section 4.6 and the results of this model compared to the experimental data.

\subsection{Total Temperature and Total Pressure Data}

Total temperature and total pressure data are presented for the flat plate at zero heat release in Section 4.1.1 and for high heat release in Section 4.1.2. Sections 4.1.3 and 4.1.4 present total temperature and total pressure data for the lobed mixer at zero and high heat releases respectively. Throughout this section, the fuel stream is the upper stream, and a trace of the trailing edge of the lobed mixer or flat plate has been overlayed on the plots. Also all total pressure data has been normalized by the average inlet dynamic pressure. 


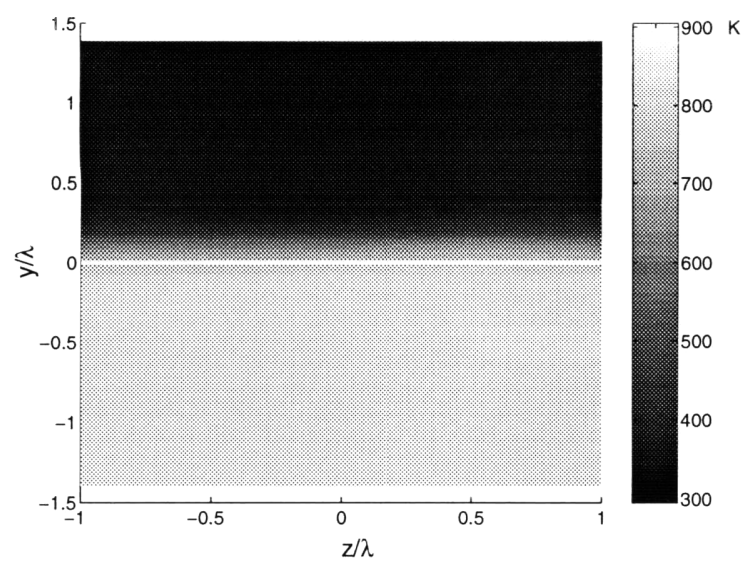

(a) $\mathrm{x} / \lambda=1.2$

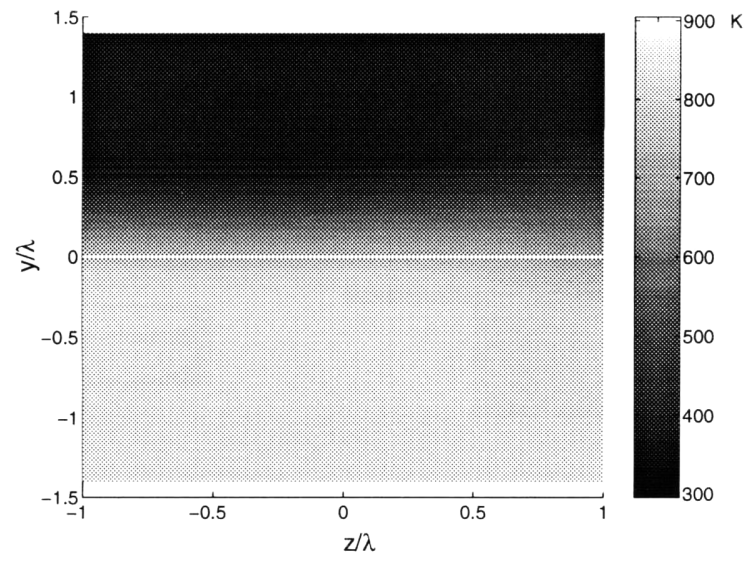

(c) $\mathrm{x} / \lambda=5.2$

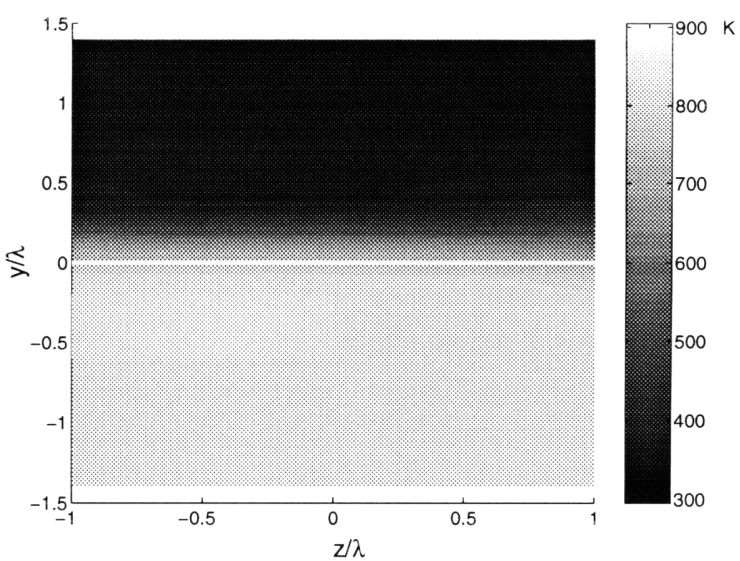

(b) $\mathrm{x} / \lambda=2.5$

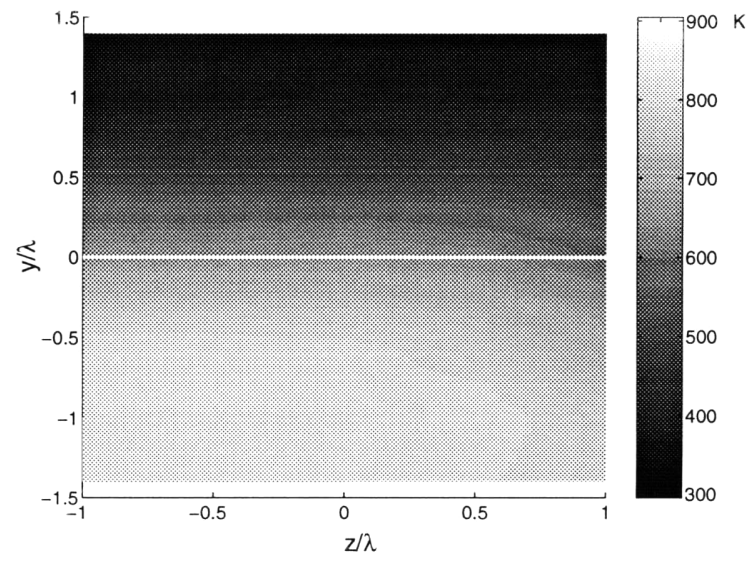

(d) $\mathrm{x} / \lambda=14.6$

Figure 4-1: Total temperature surveys for the flat plate with zero heat release $(\Phi=0)$.

\subsubsection{Flat Plate $(\Phi=0)$}

Figures 4-1 and 4-2 show total temperature and total pressure surveys for the flat plate at zero heat release. The planar reacting shear layer is seen to develop as expected with a slight deflection upward. This is due to the lighter, slower moving fuel stream on the top half of the duct. Notice that the duct has still not mixed out fully at the last downstream location, $\mathrm{x} / \lambda=14.6$. 


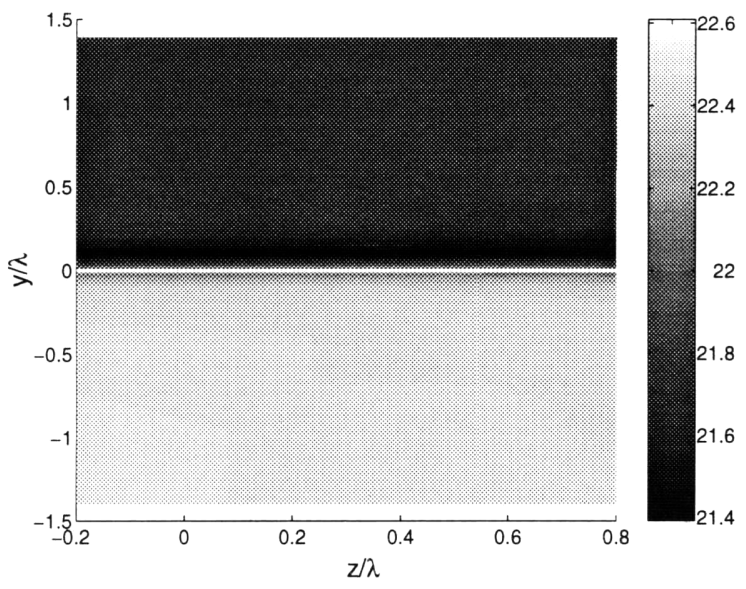

(a) $\mathrm{x} / \lambda=1.2$

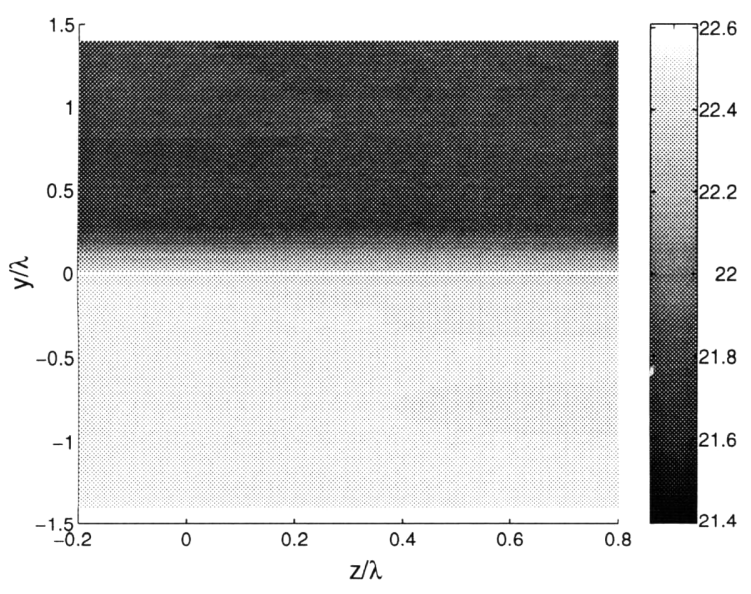

(c) $\mathrm{x} / \lambda=5.2$

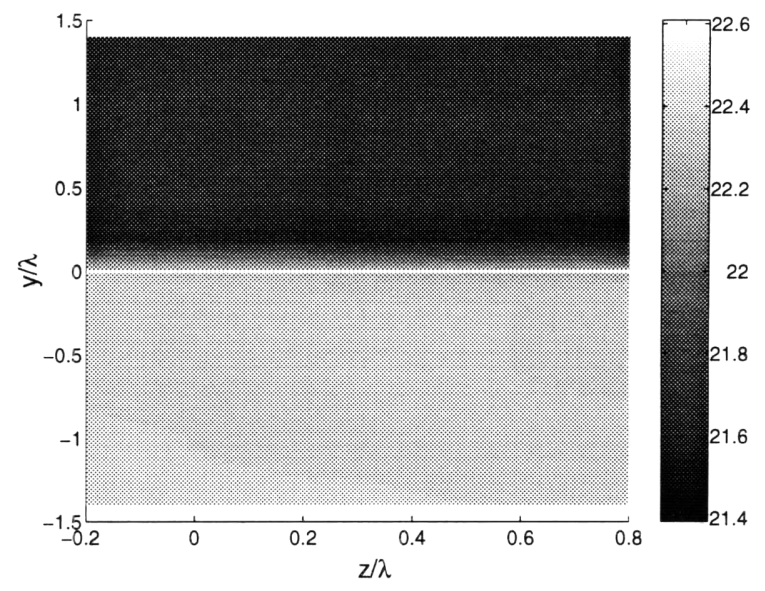

(b) $\mathrm{x} / \lambda=2.5$

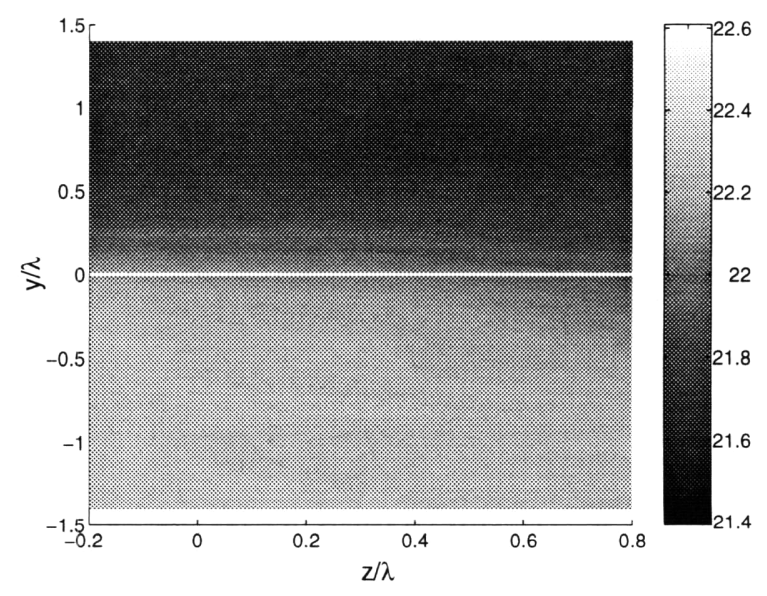

(d) $x / \lambda=14.6$

Figure 4-2: Total pressure surveys for the flat plate with zero heat release $(\Phi=0)$. 


\subsubsection{Flat Plate $(\Phi=1.84)$}

Figures 4-3 and 4-4 show total temperature and total pressure surveys for the flat plate at high heat release. The behavior of the shear layer was typical of planar reacting shear layers.[10] The slight non-uniformity on the right edge of the plots for these cases is a result of the influence of the film cooling flow. In reducing the data, averages were not taken over this region.

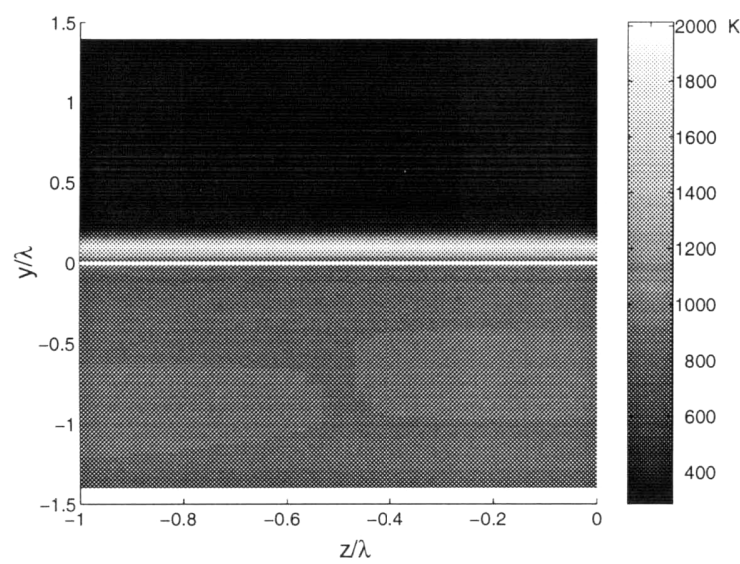

(a) $\mathrm{x} / \lambda=1.2$

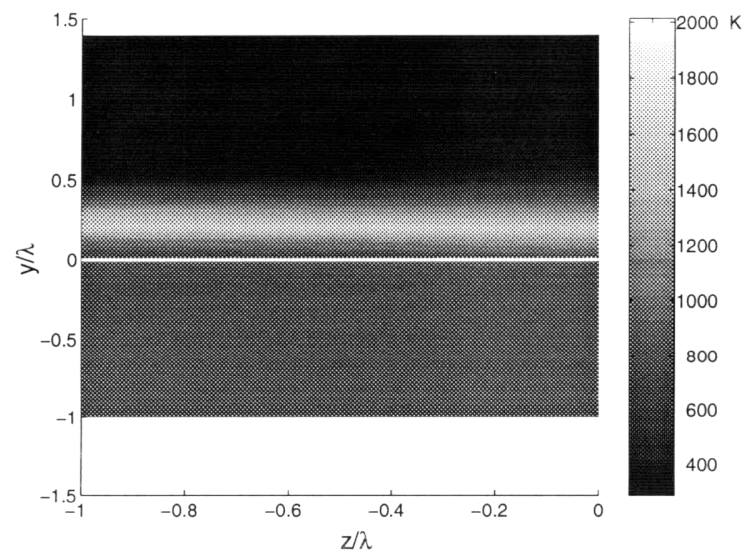

(c) $\mathrm{x} / \lambda=5.2$

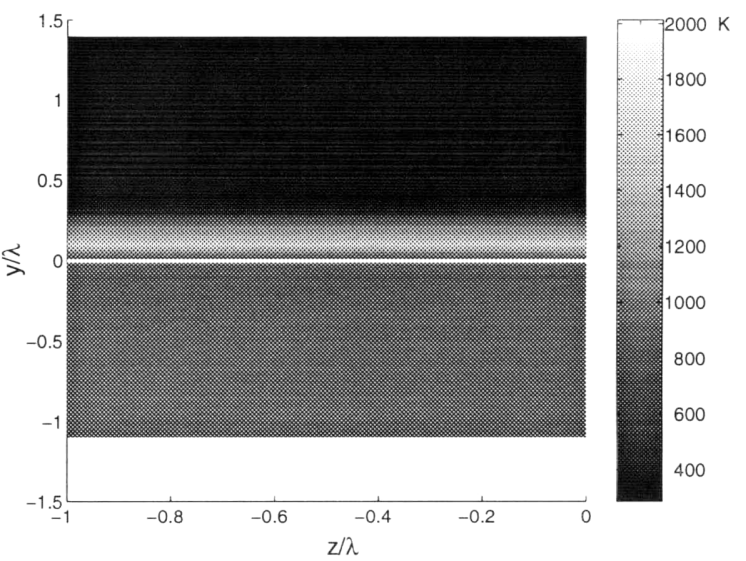

(b) $\mathrm{x} / \lambda=2.5$

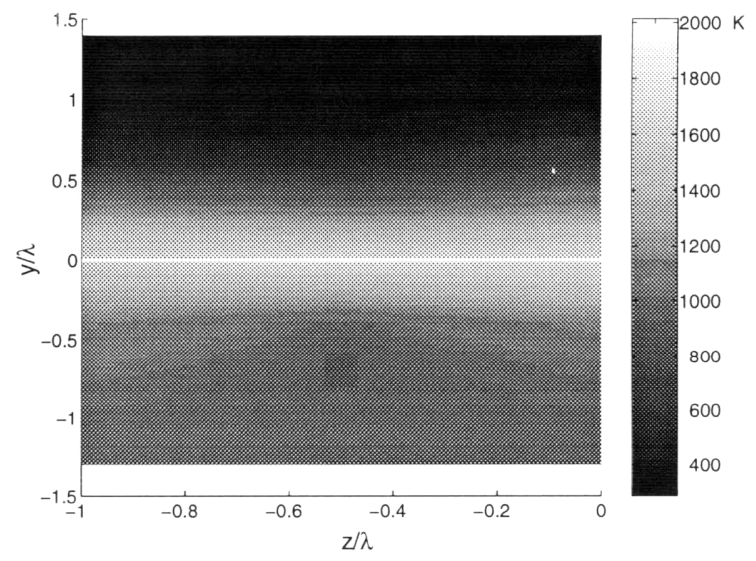

(d) $\mathrm{x} / \lambda=14.6$

Figure 4-3: Total temperature surveys for the flat plate with high heat release $(\Phi=1.84)$. 


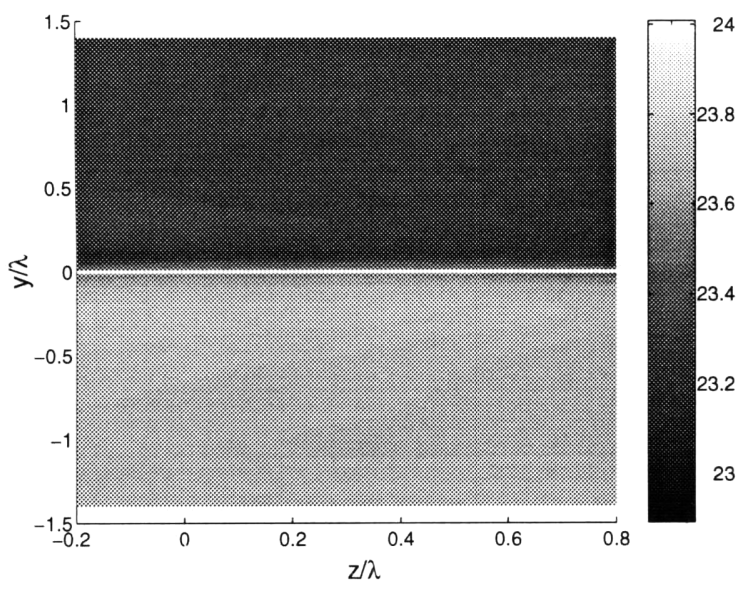

(a) $\mathrm{x} / \lambda=1.2$

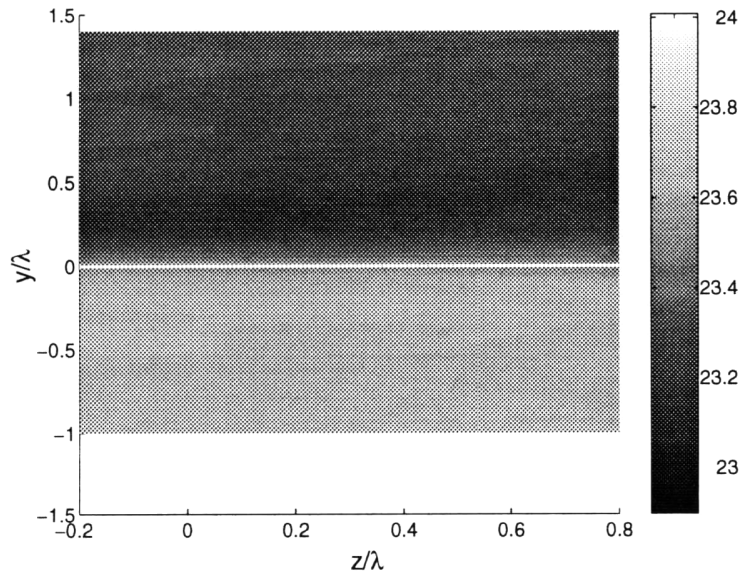

(c) $\mathrm{x} / \lambda=5.2$

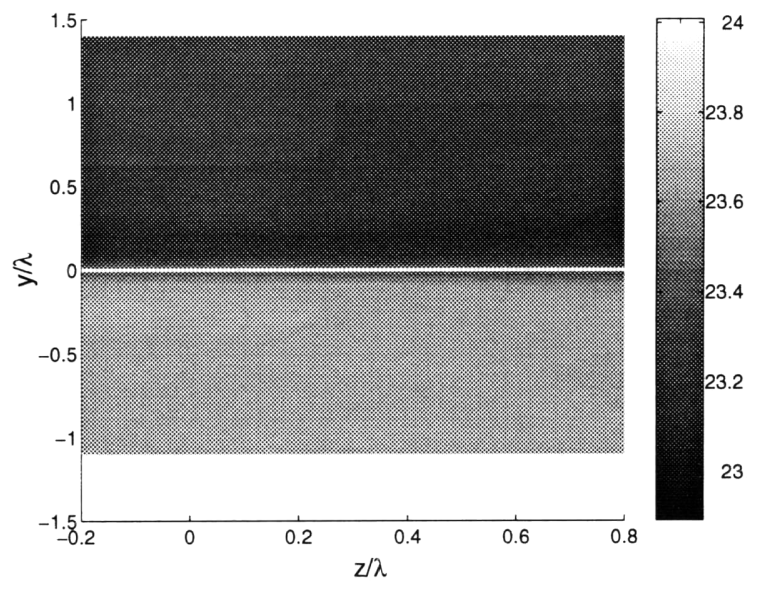

(b) $\mathrm{x} / \lambda=2.5$

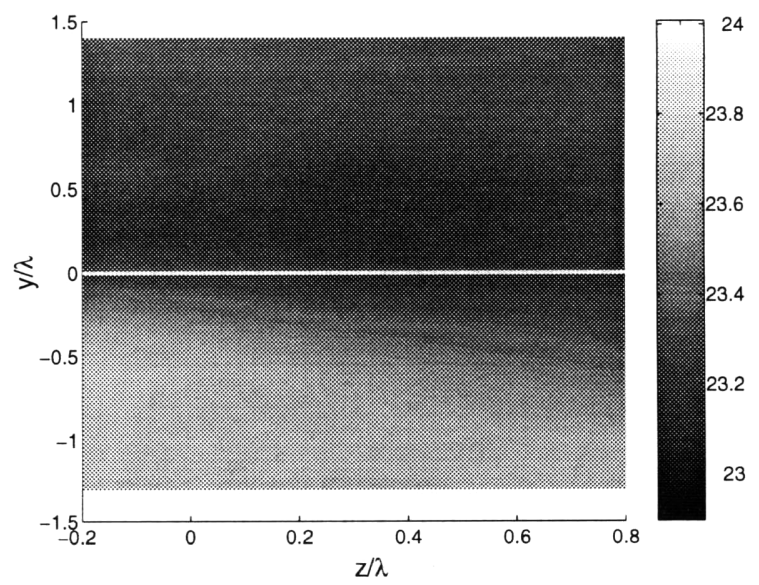

(d) $\mathrm{x} / \lambda=14.6$

Figure 4-4: Total pressure surveys for the flat plate with high heat release $(\Phi=1.84)$. 


\subsubsection{Lobed Mixer $(\Phi=0)$}

Figures 4-5 and 4-6 show total temperature and total pressure surveys for the lobed mixer at zero heat release. These results are typical of non-reacting lobed mixer flows.[2][5] Figures $4-5 \mathrm{a}$ and $4-5 \mathrm{~b}$ show that at $\mathrm{x} / \lambda=1.2$, the mixing interface has begun to roll up, showing the influence of the shed streamwise vorticity. At $\mathrm{x} / \lambda=2.5$, the interface has rolled up enough to pinch off the bottom of the fuel region (the dark area in the region $-0.5<y / \lambda<-0.1$ ). By the next downstream location, $\mathrm{x} / \lambda=5.2$, significant mixing has occurred, and the temperature and pressure profiles are more uniform, similar to those of a planar shear layer.

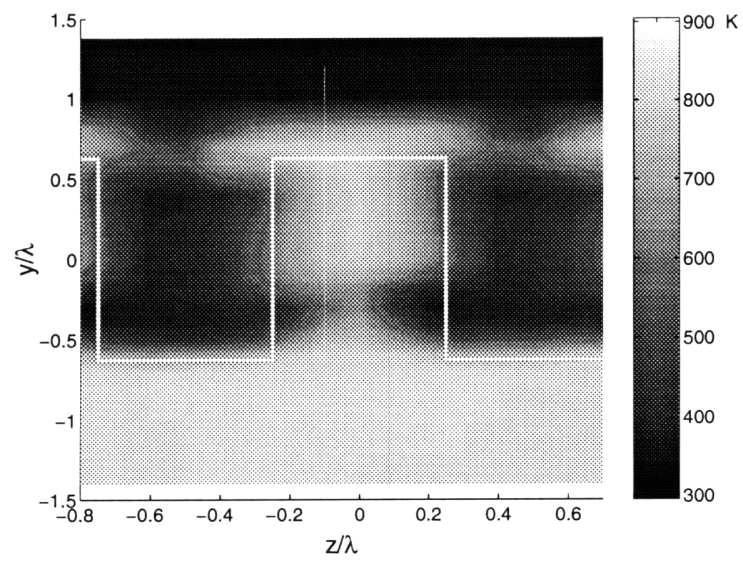

(a) $\mathrm{x} / \lambda=1.2$

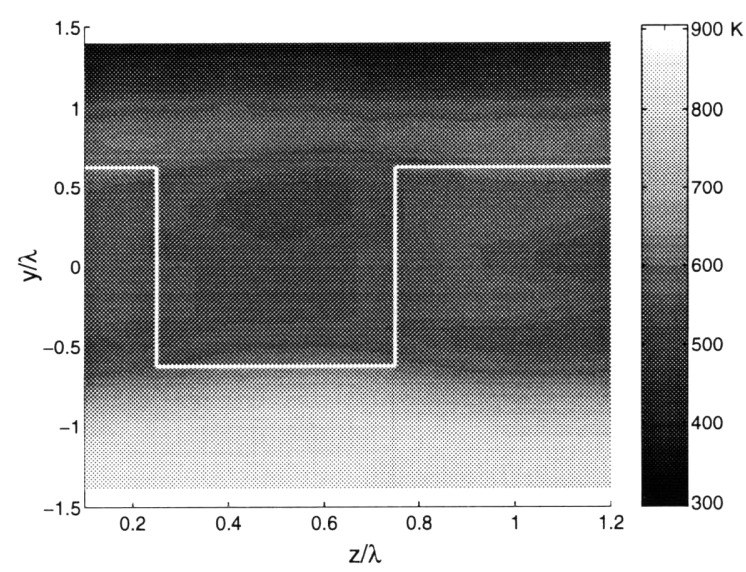

(c) $\mathrm{x} / \lambda=5.2$

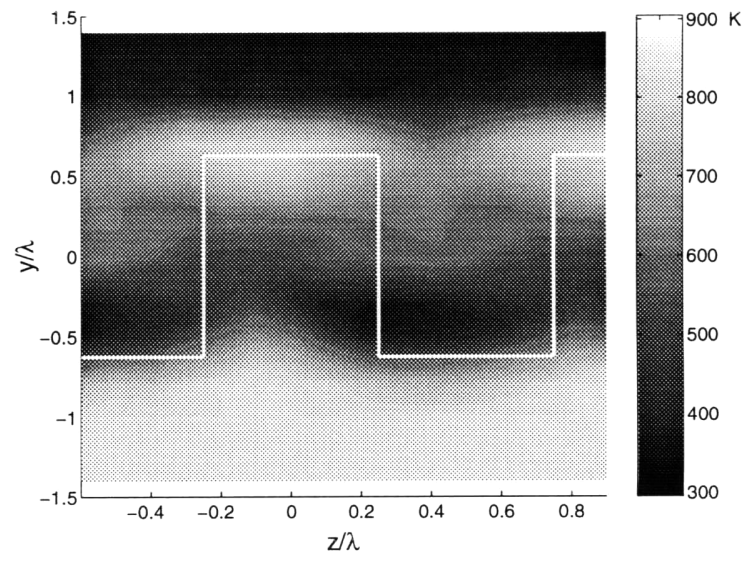

(b) $\mathrm{x} / \lambda=2.5$

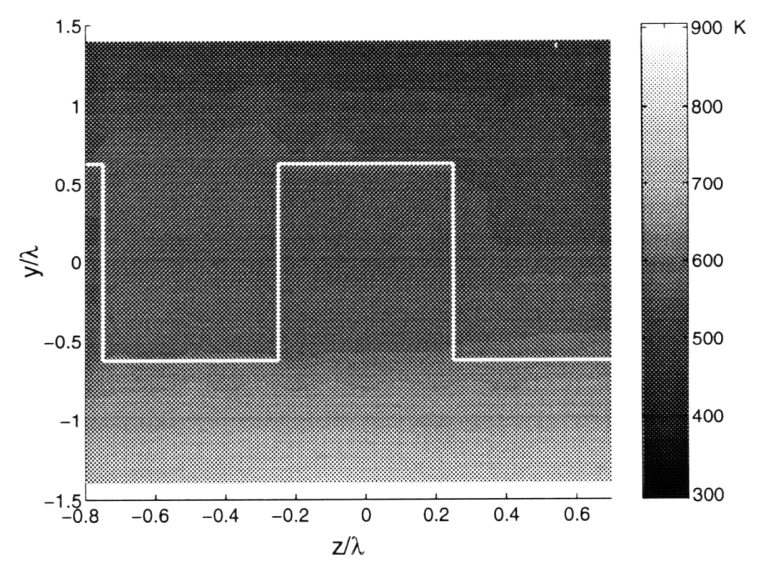

(d) $\mathrm{x} / \lambda=14.6$

Figure 4-5: Total temperature surveys for the lobed mixer with zero heat release $(\Phi=0)$. 


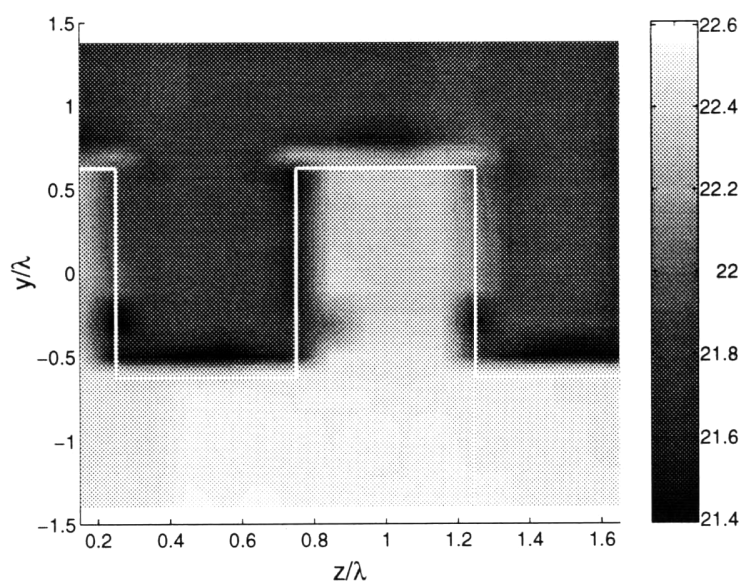

(a) $\mathrm{x} / \lambda=1.2$

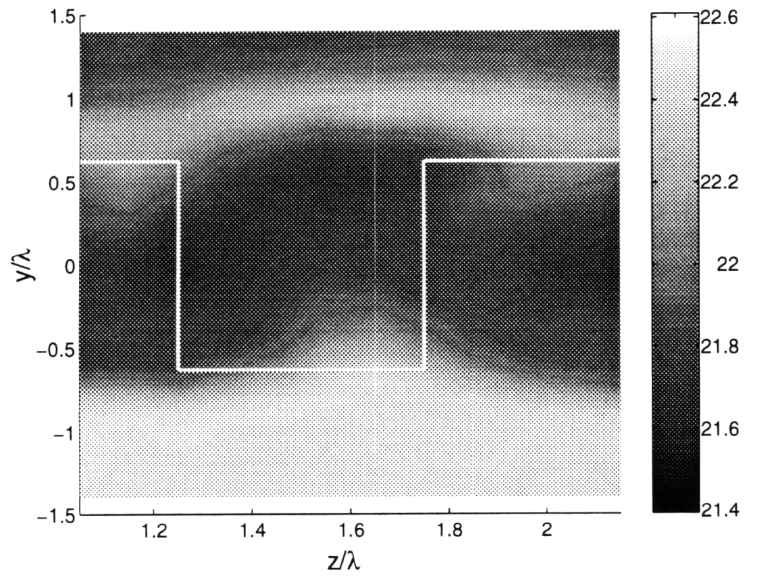

(c) $\mathrm{x} / \lambda=5.2$

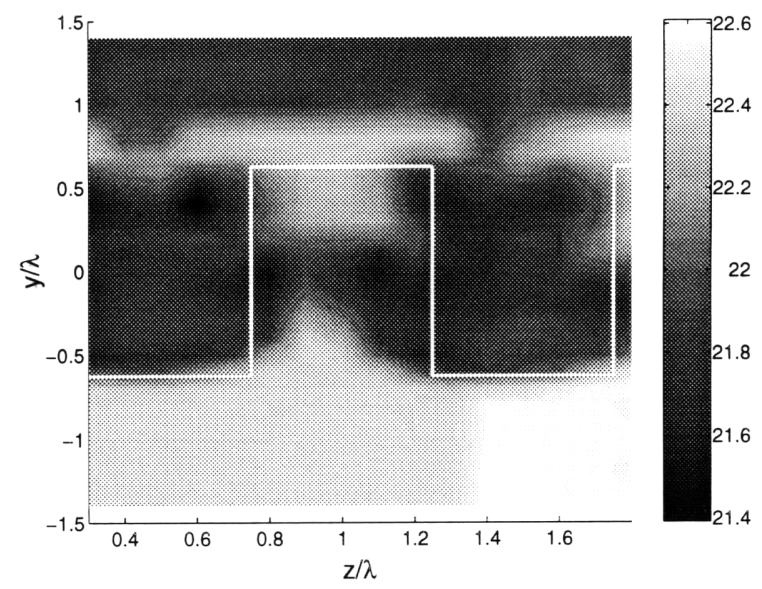

(b) $\mathrm{x} / \lambda=2.5$

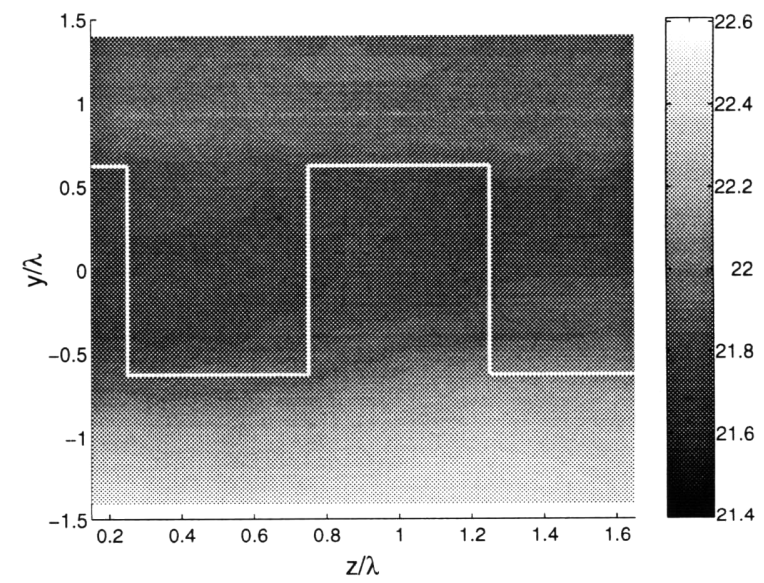

(d) $\mathrm{x} / \lambda=14.6$

Figure 4-6: Total pressure surveys for the lobed mixer with zero heat release $(\Phi=0)$. 


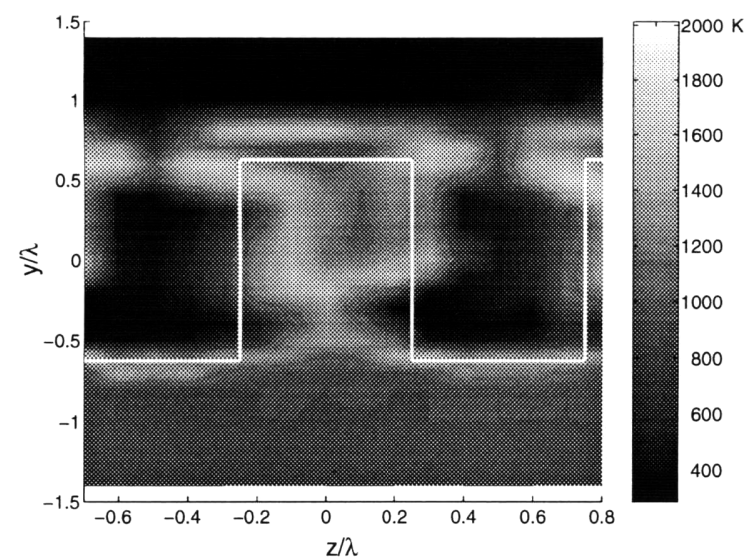

(a) $\mathrm{x} / \lambda=1.2$

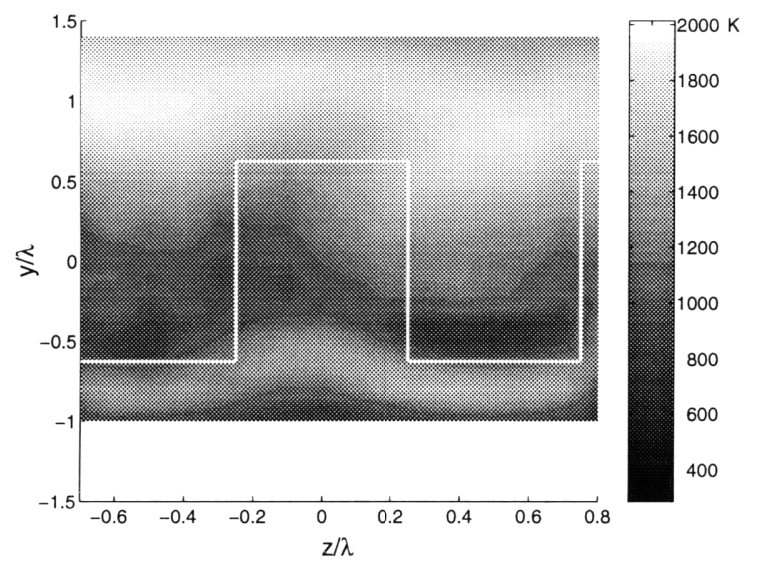

(c) $\mathrm{x} / \lambda=5.2$

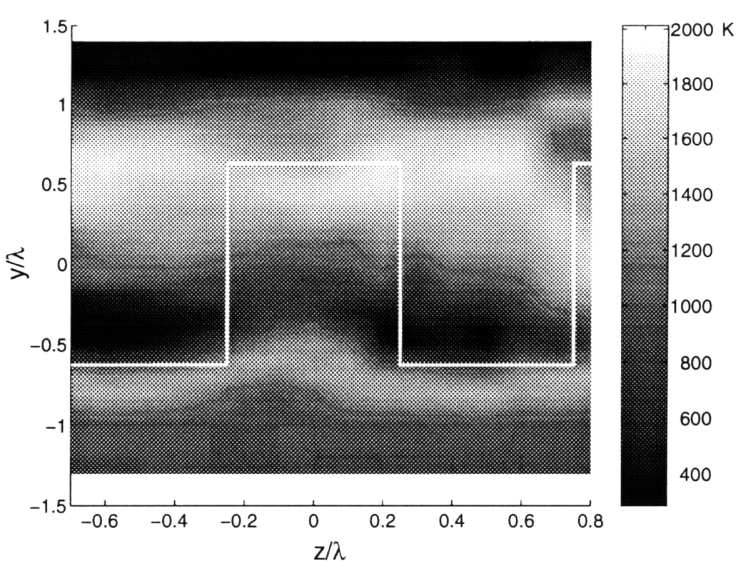

(b) $\mathrm{x} / \lambda=2.5$

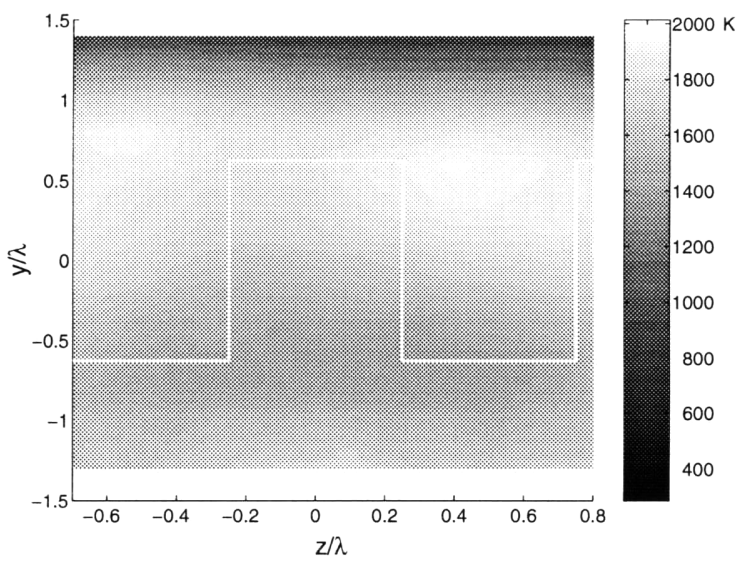

(d) $\mathrm{x} / \lambda=14.6$

Figure 4-7: Total temperature surveys for the lobed mixer with high heat release $(\Phi=1.84)$.

\subsubsection{Lobed Mixer $(\Phi=1.84)$}

Figures 4-7 and 4-8 show total temperature and total pressure surveys for the lobed mixer at high heat release. The flow structure shown is very similar to that presented previously in Section 4.1.3 for the non-reacting lobed mixer. Regions of fuel are pinched off by $\mathrm{x} / \lambda=2.5$, and nearly complete mixing has occurred by $\mathrm{x} / \lambda=14.6$. The significant mixing and heat release can be compared to the flat plate shown previously in Figures 4-3 and 4-4. 


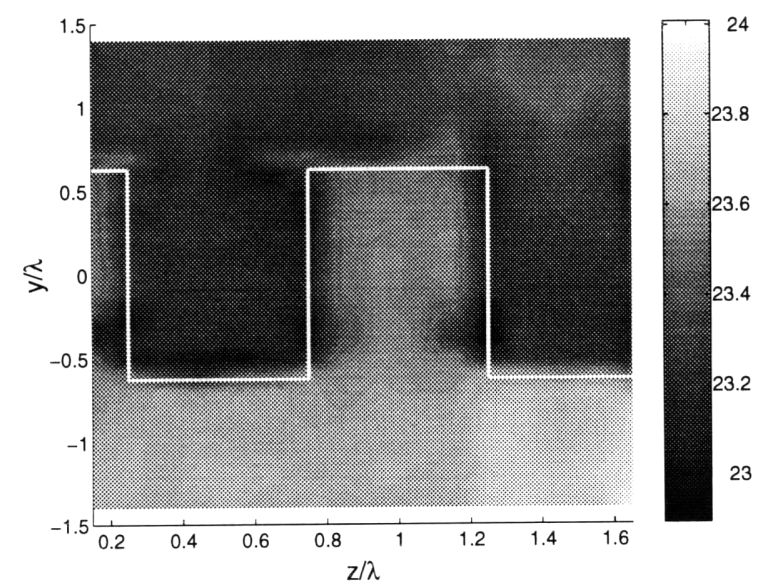

(a) $\mathrm{x} / \lambda=1.2$

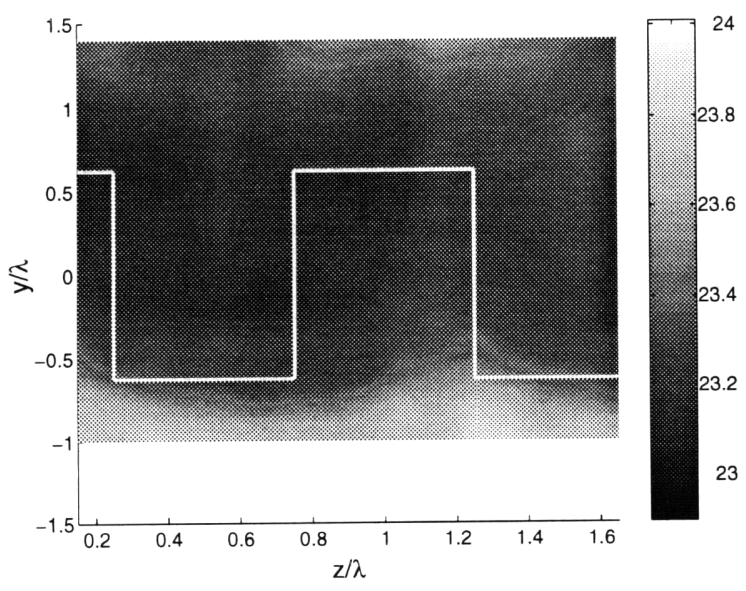

(c) $\mathrm{x} / \lambda=5.2$

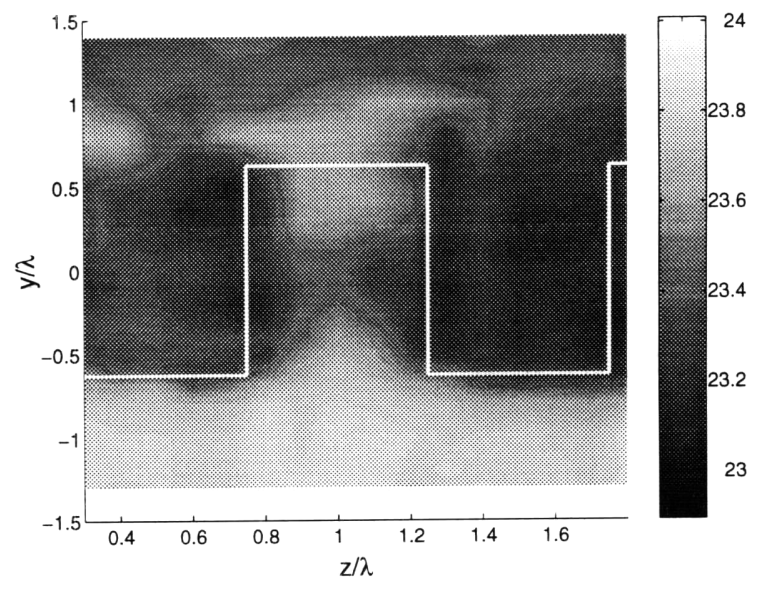

(b) $\mathrm{x} / \lambda=2.5$

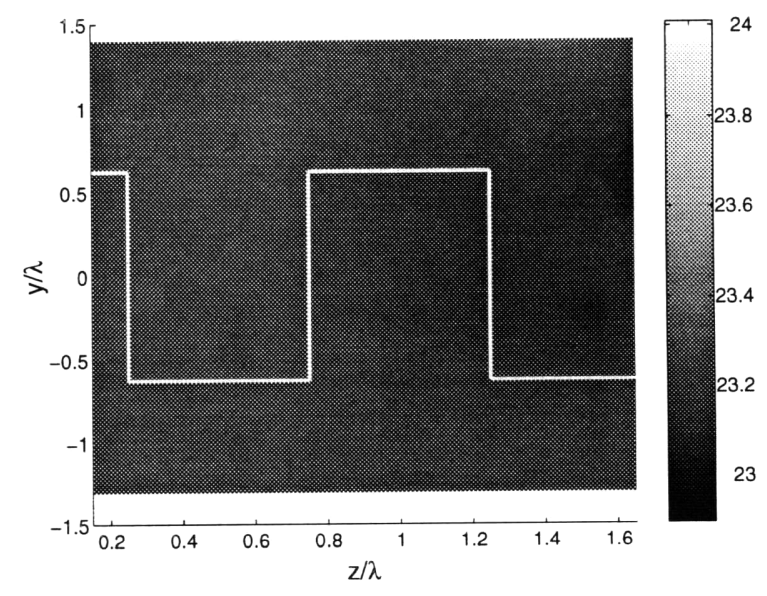

(d) $\mathrm{x} / \lambda=14.6$

Figure 4-8: Total pressure surveys for the lobed mixer with high heat release $(\Phi=1.84)$. 


\subsection{Losses}

There are two primary sources of total pressure loss in lobed mixer and flat plate flows. The first is the skin friction loss over the lobes or flat plate. The second is the loss due to the mixing out of velocity non-uniformities downstream of the trailing edge.

The skin friction losses over the lobed mixer and flat plate were compared to those calculated by a three-dimensional Navier-Stokes code run at the same conditions as those described in Section 3.1. The loss coefficient, $\mathrm{L}$, is defined as

$$
L=\frac{\bar{p}_{t, i n}-\bar{p}_{t}}{\bar{q}_{i n}}
$$

where $\bar{p}_{t, \text { in }}$ is the mass averaged total pressure at the beginning of the lobes or flat plate, $\bar{p}_{t}$ is the mass averaged total pressure at the trailing edge, and $\bar{q}_{i n}$ is the average dynamic pressure at the entrance to the lobes. The measured and computed loss coefficients for the lobed mixer were equal, while they were almost equal for the flat plate. The results of this comparison are shown in Table 4.1. The skin friction losses over the lobed mixer were approximately five times greater than those over the flat plate, yet they were still only $7 \%$ of the average dynamic head.

The losses for the flat plate and lobed mixer downstream of the trailing edge were also compared to the results of the Navier-Stokes code. The loss coefficient is defined as before in Equation 4.1 where $\bar{p}_{t, \text { in }}$ is the mass averaged total pressure at the trailing edge, $\bar{p}_{t}$ is the mass averaged total pressure at a given downstream location, and $\bar{q}_{\text {in }}$ is the average dynamic pressure at the trailing edge. The results of this comparison

Table 4.1: Experimental and computational loss coefficients, L, for the lobed mixer and flat plate.

\begin{tabular}{|c|c|c|}
\hline Geometry & Experimental & Computational \\
\hline Flat Plate & 0.015 & 0.014 \\
\hline Lobed Mixer & 0.070 & 0.070 \\
\hline
\end{tabular}




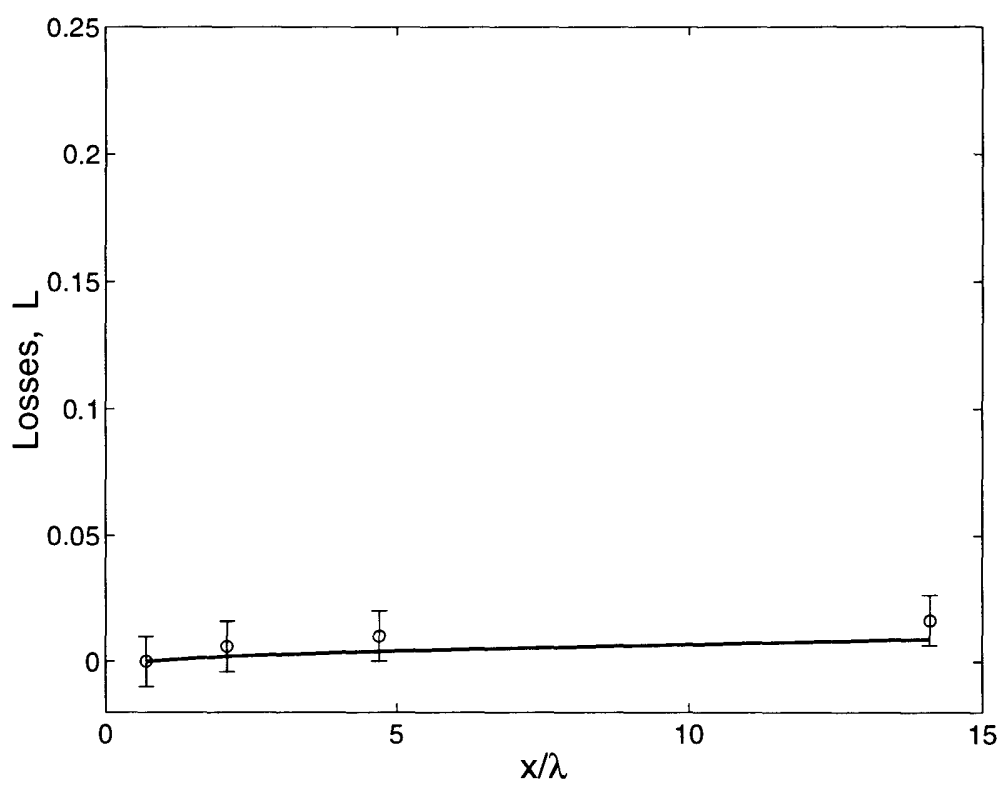

(a) flat plate

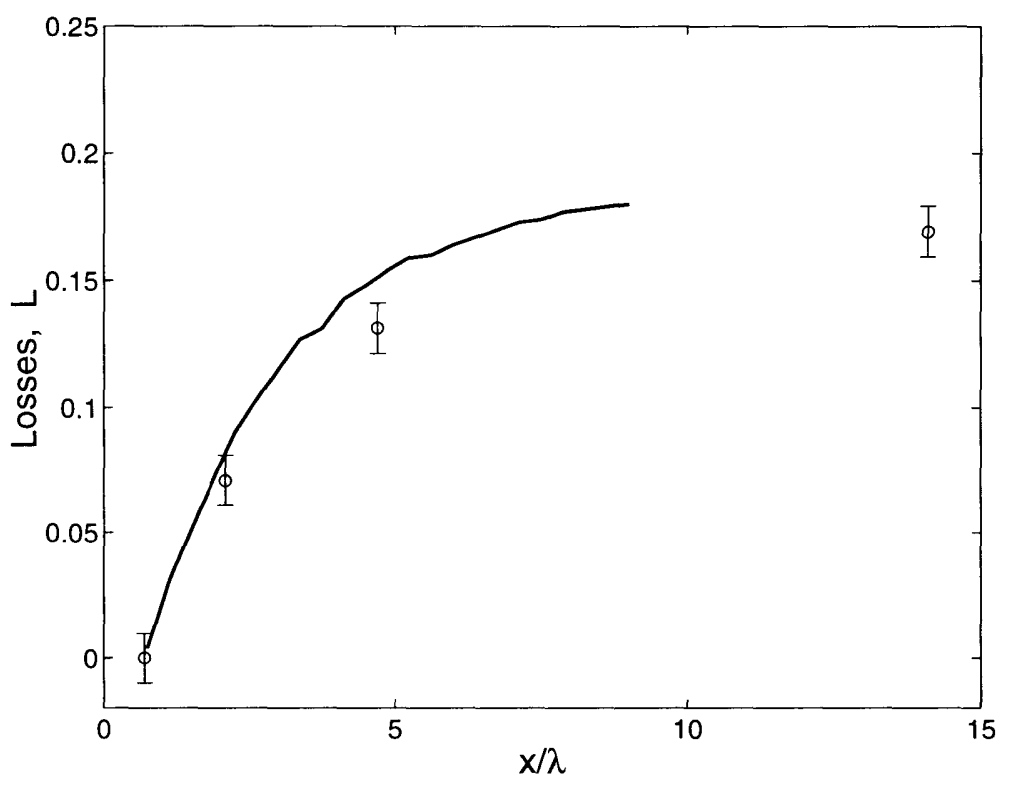

(b) lobed mixer

Figure 4-9: Experimental (o) and computational (line) loss coefficients for (a) flat plate and (b) lobed mixer. 
are shown in Figure 4-9. The circles represent the experimental data and the lines represent the results of the Navier-Stokes code. These results show that the mixing losses are greater for the lobed mixer than the flat plate, as expected, indicating more mixing for the lobed mixer. The agreement for the flat plate is good. The code over estimated the losses for the lobed mixer by approximately $25 \%$. This coincided with an initial mixing rate which was approximately $25 \%$ higher for the computational case.

\subsection{Static Pressure and Total Enthalpy Profiles}

Figures 4-10 and 4-11 show experimental static pressure, $C_{p}$, and mass-averaged total enthalpy, $C_{h t}$, measurements. The lines represent the results of the control volume model discussed in Section 3.2. The static pressure has been matched to the experimental data by adjusting the primary mixing rate. The static pressure rise coefficient, $C_{p}=\Delta p /\left(\bar{\rho} \bar{u}^{2} / 2\right)$, is defined as before. The total enthalpy rise coefficient is defined as $C_{h t}=\Delta \bar{h}_{t} / \bar{h}_{t, \text { inlet }}$. The error bars shown on Figures 3-5, 4-10, and 4-11 were obtained by estimating the random and bias errors in each of the measured quantities. The errors were then propagated through the calculation of $C_{p}$ and $C_{h t}$ and plotted as $95 \%$ confidence bands.

Comparison of Figures 4-10a and 4-11a shows that for zero heat release, the static pressure rise for the lobed mixer was almost double that of the flat plate. This result is typical of flows generated by lobed mixers with the geometry described in Section 2.[5] Comparing Figures 4-10a and 4-11a with Figures 4-10c and 4-11c, it is evident that the addition of heat release changed the static pressure profiles markedly. Although, a pressure rise due to the mixing still existed, the pressure drop due to the combustion process more than counteracted this, yielding a net reduction in pressure. The exit pressure coefficient for the lobed mixer varied from +0.45 with zero heat release to -1.05 with high heat release. Also notice that, as expected, the enthalpy rise increased as $\Phi$ increased.

Comparison of Figures $4-10 \mathrm{~b}$ and $4-11 \mathrm{~b}$ shows that for the low heat release case 

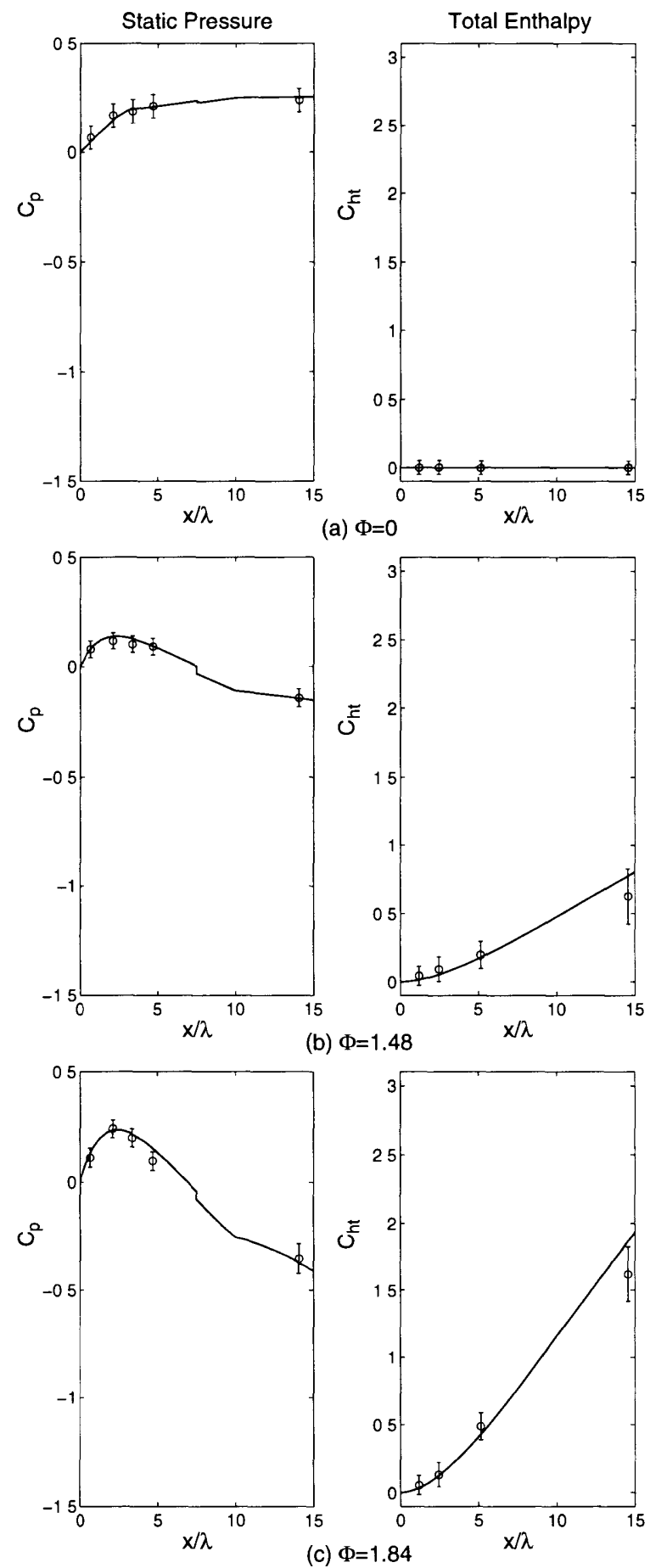

Figure 4-10: Flat plate experimental (o) and computed (line) static pressure and total enthalpy profiles for (a) $\Phi=0$, (b) $\Phi=1.48$, and (c) $\Phi=1.84$. 

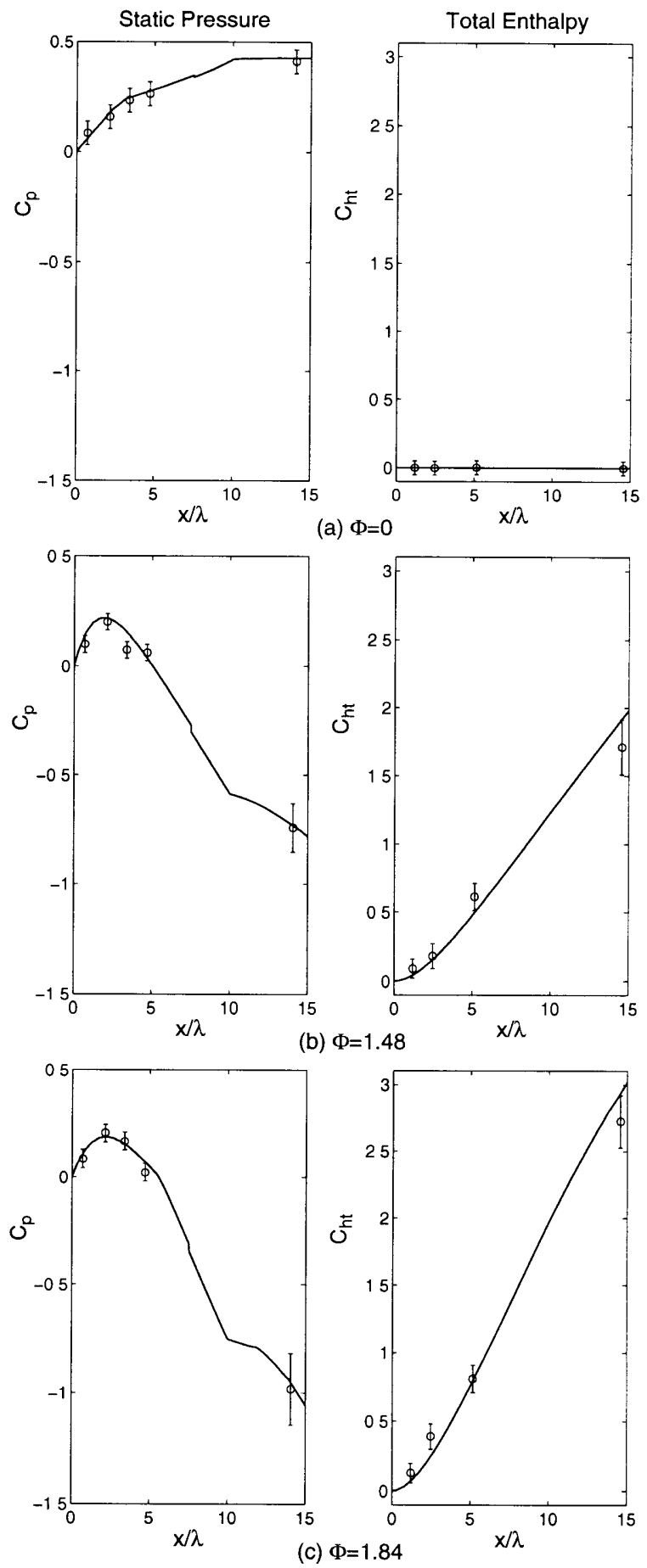

Figure 4-11: Lobed mixer experimental (o) and computed (line) static pressure and total enthalpy profiles for (a) $\Phi=0$, (b) $\Phi=1.48$, and (c) $\Phi=1.84$. 
$(\Phi=1.48)$, the mixing augmentation due to the lobed mixer produced almost four times the pressure drop produced by the flat plate, and the enthalpy rise was more than double. This effect is seen again in Figures 4-10c and 4-11c for the high heat release case $(\Phi=1.84)$, where the pressure drop was increased by a factor of 2.5 , and the enthalpy rise was increased by a factor of 1.5 .

The sensitivity of the derived mixing rate to changes in static pressure or massaveraged total enthalpy was also examined. Mixing rate was varied in the control volume model to match either static pressure or total enthalpy to the upper and lower points of the error bars shown in Figures 4-10 and 4-11. For all of the experimental cases, the change in mixing rate was greater when static pressure, not mass-averaged total enthalpy, was matched at the high and low points of the error bars. Thus the mixing rate determination was more sensitive to static pressure than total enthalpy. Therefore, throughout this study, static pressure was matched in order to determine mixing rate. Details of the sensitivity study are presented in Appendix B.

\subsection{Mixing Rates and Mixedness}

Scalar mixedness was used as a measure of species transport. This mixedness parameter is defined as

$$
M=\frac{\dot{m}_{m i x}}{\dot{m}_{i n}}
$$

where $\dot{m}_{m i x}$ is the mass flow rate of products in the mixing layer, and $\dot{m}_{i n}$ is the mass flow rate of reactants in the experimental survey plane $(-1.5<y / \lambda<1.5$ and $-0.7<z / \lambda<0.8)$ at the inlet. The value of $\mathrm{M}$ can vary from 0 at the trailing edge, representing two completely unmixed fluid streams, to 1 far downstream, representing two completely mixed fluid streams. No film cooling air was present in the surveyed regions, therefore, the film cooling mass flow rate was not included in the calculation of $\dot{m}_{i n}$. 
The effect of heat release on mixedness is shown in Figure 4-12. The shaded areas represent the effect on the calculated mixedness if the static pressure profiles were matched through the upper and the lower points of the experimental error bars as discussed earlier in Section 4.3. The line in the middle of each shaded region is the best estimate of the mixing rate. This being the mixing rate which produced the static pressure profiles shown in Figures 4-10 and 4-11. From Figure 4-12, the mixing rate, given by the slope of the mixedness curve, can be seen to decrease with increasing heat release.

As shown in Figure 4-12a the flat plate mixing rate decreased by a factor of 4 for the high heat release case $(\Phi=1.84)$ compared to the flat plate mixing rate for the zero heat release case. Comparing Figures $4-12 \mathrm{a}$ and $4-12 \mathrm{~b}$, it can also be seen that the lobed mixer initially mixed approximately 6 times faster than the flat plate at zero heat release and approximately 12 times faster at high heat release. The initial mixing rate for the lobed mixer was decreased by a factor of 2 from zero to high heat release, while the mixing rate in the far field of the lobed mixer for the high heat release case was decreased by a factor of 3.6 compared to the zero heat release case. Thus in the near field of the lobed mixer $(x / \lambda \leq 3)$, the mixing rate was less sensitive than that of the flat plate flow to the detrimental effects of heat release.

The higher mixing rate for the lobed mixer increased the level of mixedness at the last survey plane for the zero heat release case by a factor of 3 over the flat plate mixedness at zero heat release, while the final mixedness for the lobed mixer at high heat release was greater than that of the flat plate at high heat release by a factor of 5 . The mixedness of the lobed mixer flow at the last measurement plane was decreased by a factor of 2.3 for the high heat release case compared to the lobed mixer with zero heat release. Note that the change in the lobed mixer mixing rate with heat release in the far field was approximately proportional to the change in the flat plate mixing rate.

Hermanson[11] has suggested that in order to ensure that there are no trailing edge wake effects in the measurements and to ensure that the mixing layer is selfsimilar, measurements in planar shear layers should be taken at least 1000 momentum 


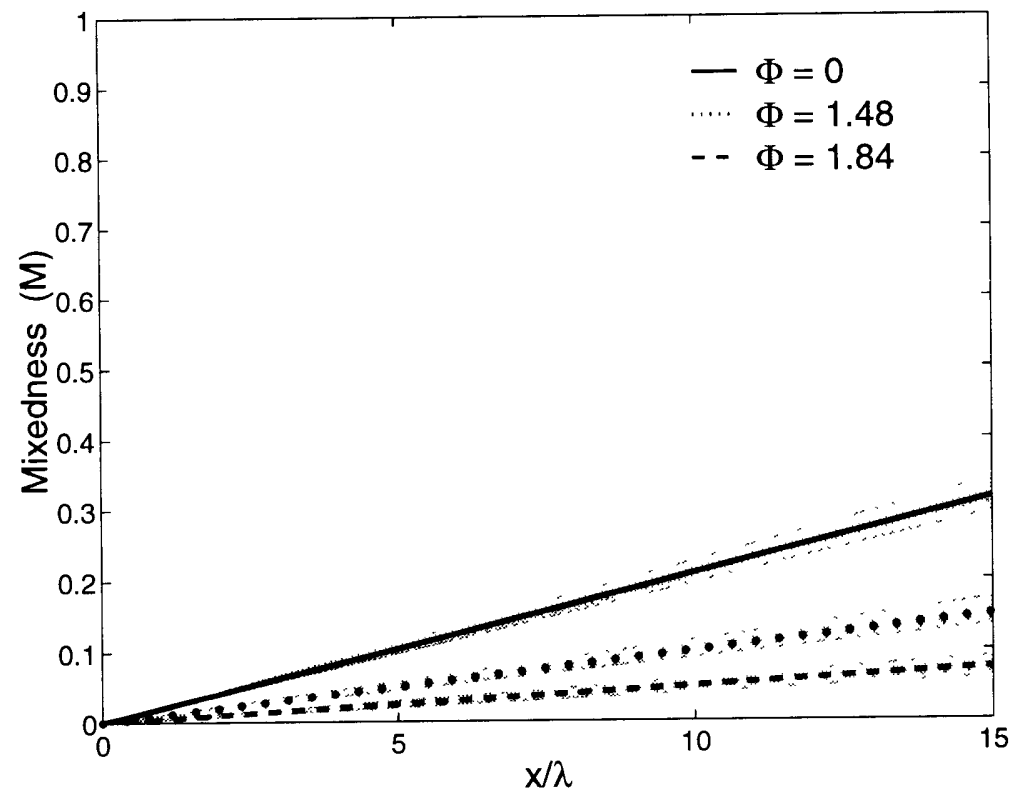

(a) flat plate

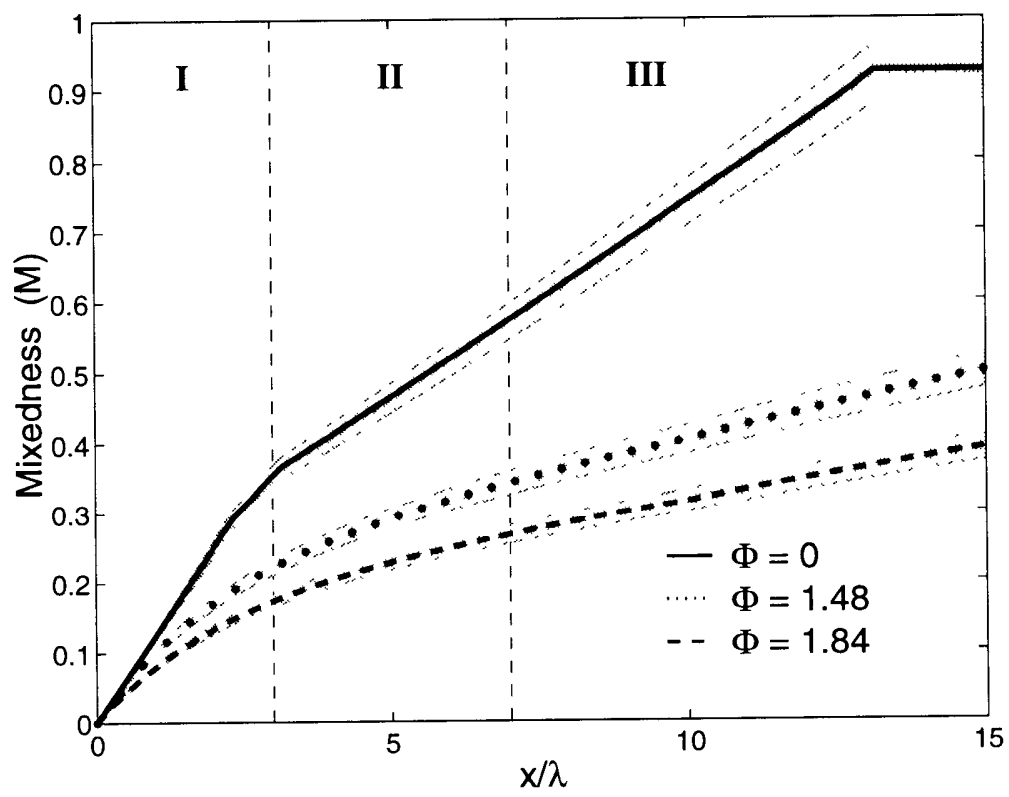

(b) lobed mixer

Figure 4-12: Mixedness curves with error bands for (a) flat plate and (b) lobed mixer. 
thicknesses downstream of the trailing edge. It it also desirable to be above the Reynolds number for mixing transition to ensure a turbulent mixing layer. The Reynolds number based on the high speed free stream velocity and the downstream distance was typically 950,000 . This was well above the the Reynolds number for mixing transition as reported by Konrad[12].

The momentum thickness at the trailing edge of both the flat plate and lobed mixer were computed. The flat plate boundary layer thickness was calculated by taking the boundary layer thickness to be zero at the throat of the contraction from the plenum to the test section inlet and using the relation for boundary layer growth over a flat plate for the 5 inches between the throat and the trailing edge. This yielded a boundary layer thickness of $3 \mathrm{~mm}$. Assuming a $1 / 7$ power law velocity profile, the momentum thickness was then taken to be an eighth of the boundary layer thickness, yielding a momentum thickness of $0.375 \mathrm{~mm}$. The lobed mixer boundary layer thickness was calculated by using the flat plate relation for the three-quarters of an inch between the throat and beginning of the lobes. This boundary layer thickness was 2.5 times thinner than the thinnest boundary layer thickness used in the Navier-Stokes code[9] described in Sections 3.1 and 4.2. Therefore, the boundary layer thickness at the trailing edge was conservatively estimated using results from the numerical simulation. For the lobed mixer, the boundary layer varied between 0.2 $\mathrm{mm}$ and $1.9 \mathrm{~mm}$, and the average boundary layer thickness was $1.05 \mathrm{~mm}$. Assuming a $1 / 7$ power law velocity profile, the average momentum thickness for the lobed mixer was $0.13 \mathrm{~mm}$, or $0.4 \%$ of the lobe height.

The flat plate boundary layer corresponded to 68 momentum thicknesses per lobe wavelength, while the average lobed mixer boundary layer was thinner at 195 momentum thicknesses per lobe wavelength. Thus the measurements were taken upstream of the 1000 momentum thickness guideline suggested by Hermanson[11]. However, the mixing rate data in Figure 4-12a shows that the planar shear layer displayed a linear growth rate for $\mathrm{x} / \lambda \leq 1$. This implies that at the first measurement plane, $\mathrm{x} / \lambda=1.2$, the flow behavior was similar to a fully developed turbulent shear layer. Since the lobed mixer had almost 3 times the number of momentum thicknesses per 
lobe wavelength as the flat plate, it was assumed that the mixing region downstream of the lobed mixer was also turbulent and fully developed.

\subsection{Mixing Augmentation With Heat Release}

The lobed mixer mixing rate had three regions where the behavior was different. Figure $4-12 \mathrm{~b}$ shows these regions. In region I, streamwise vorticity had a dominant effect. In region III, comparison with numerical simulations[3] and the cold flow experiments of McCormick[2] suggests that the streamwise vorticity had decayed, and was no longer having a strong influence on the mixing process. In region II, the behavior was some combination of these two cases.

The focus will first be on region I where streamwise vorticity had a dominant effect. Two contributions to mixing rate were identified and evaluated for the lobed mixer flow. The first was the increased mixing interface length due to the increased trailing edge length of the lobed mixer. This will be called the interface-scaled planar shear layer mixing rate, and will be denoted as $\ell d M_{p s l}$ where $\ell$ is the lobed mixer to flat plate trailing edge length ratio, and $d M_{p s l}$ is the planar shear layer mixing rate with the denominator,$d(x / \lambda)$, dropped for convenience, but implied. The second contribution was the additional mixing associated with the shed streamwise vorticity. This increase in mixing rate will be denoted by $d M_{a}$ and will be called the augmented mixing rate. Thus, the overall mixing rate of the lobed mixer is given by

$$
d M_{t o t}=d M_{a}+\ell d M_{p s l}
$$

where $d M_{t o t}$ is the slope of the lobed mixer mixedness curves shown in Figure $4-12 \mathrm{~b}$.

The experimental mixing augmentation rates were found by subtracting the interfacescaled planar shear layer mixing rate, $\ell d M_{p s l}$, from the total mixing rate, $\mathrm{d} M_{t o t}$, as suggested in Equation 4.3. For these experiments, $\ell=3.2$. The values for the initial lobed mixer mixing rates were calculated by averaging the slope of the mixedness 
Table 4.2: Flat plate and lobed mixer mixing rates.

\begin{tabular}{|c|c|c|c|}
\hline Heat Release, $\Phi$ & $d M_{p s l}$ & $\ell d M_{p s l}$ & $d M_{\text {tot }}$ \\
\hline 0 & 0.021 & 0.054 & 0.117 \\
\hline 1.48 & 0.010 & 0.025 & 0.073 \\
\hline 1.84 & 0.005 & 0.015 & 0.057 \\
\hline
\end{tabular}

Table 4.3: Observed mixing augmentation rates, $d M_{a}$.

\begin{tabular}{|c|c|}
\hline Heat Release, $\Phi$ & $d M_{a}$ \\
\hline 0 & 0.050 \\
\hline 1.48 & 0.041 \\
\hline 1.84 & 0.041 \\
\hline
\end{tabular}

curves over the first three wavelengths. This distance was used based on the results of Fung[3] and McCormick[2]. Fung identified a decay point defined by

$$
\frac{x_{\text {decay }}}{\lambda} \sim \frac{1+r}{1-r}
$$

which yields an $x_{\text {decay }} / \lambda$ of 3 . This decay point is assumed to be where the mixing augmentation associated with the streamwise vorticity becomes insignificant. Further, McCormick's data for the circulation decay of a lobed mixer showed that the circulation drops to half its initial value by 3.5 lobe wavelengths.

Table 4.2 shows the values of the overall mixing rates observed for a flat plate and lobed mixer, while Table 4.3 shows the derived values for the augmented mixing rate. These results show that the mixing augmentation due to streamwise vorticity was reduced approximately $18 \%$ by heat release. This reduction may be compared to the $76 \%$ reduction displayed in the flat plate data shown in Figure 4-12a.

Table 4.4 compares the mixing rates for the flat plate at zero, low, and high heat releases as shown in Figure 4-12a with the results of Hermanson and Dimotakis[10] for a flat plate with the same heat releases. The results for the flat plate agree within 
Table 4.4: Comparison of flat plate mixing rates for varying heat release with the results of Hermanson and Dimotakis.

\begin{tabular}{|c|c|c|}
\hline Heat Release, $\Phi$ & Present Study & Hermanson and Dimotakis \\
\hline 0 & 0.021 & 0.019 \\
\hline 1.48 & 0.010 & 0.009 \\
\hline 1.84 & 0.005 & 0.006 \\
\hline
\end{tabular}

$10 \%$ with those of Hermanson and Dimotakis.

\subsection{Analytical Model}

The mixing rate augmentation due to the addition of streamwise vorticity was expected to decrease with increasing heat release based on the results of a model developed by Karagozian and Marble[14] for the mixing augmentation due to an isolated vortex with heat release. The Karagozian and Marble model is described below followed by a comparison of the augmented mixing rates predicted by this model with those measured.

The model has several assumptions. The fuel and oxidizer are each assumed to fill a semi-infinite half plane, and the reaction is assumed to have fast chemical kinetics such that the reaction is diffusion controlled. This is a valid assumption in this experiment since the hydrogen-air reaction mechanism possesses kinetic time scales on the order of $10^{-5}$ seconds while the residence time in the test section is on the order of $10^{-2}$ seconds. The vortex is assumed to be isolated on the interface of the two reactant half-planes. This possibly is not a good assumption for the current experiments since by 2 lobe wavelengths downstream of the trailing edge the vortices had grown together. Another assumption is that the radius of the burned core greatly exceeds that of the viscous core and therefore the field may be treated as inviscid. The final assumption is that the dominant effect of the reaction is to reduce the gas density in the core.

The scaling developed by Karagozian and Marble for the vortex mixing rate augmentation with a change in density is 


$$
\frac{d M_{a}}{d(x / \lambda)} \sim \frac{\Gamma^{\frac{2}{3}} D_{T}^{\frac{1}{3}}}{\bar{U} \lambda} \frac{1}{\pi} \int_{0}^{\beta^{*}}\left\{[J(\beta)]^{-\frac{1}{2}}\left(\frac{\left(\frac{D_{T}}{\Gamma}\right)^{2}+\beta^{2}}{1+\frac{\beta}{\beta^{*}}\left(\frac{\rho_{1}}{\rho_{2}}-1\right)}\right)-\left(\frac{D_{T}}{\Gamma}\right)^{\frac{1}{3}} \beta^{-\frac{1}{2}}\right\} \frac{d \beta}{\beta}
$$

where

$$
J(\beta) \equiv \int_{0}^{\beta} \frac{\left(\frac{D_{T}}{\Gamma}\right)^{\frac{2}{3}}+\beta^{2}}{1+\frac{\beta}{\beta^{*}}\left(\frac{\rho_{1}}{\rho_{2}}-1\right)} d \beta
$$

and

$$
\beta \equiv \frac{\Gamma^{\frac{2}{3}} D_{T}^{\frac{1}{3}} t}{\pi \xi^{2}} \quad \beta^{*} \equiv \frac{\Gamma^{\frac{2}{3}} D_{T}^{\frac{1}{3}} t}{\pi \xi *^{2}}
$$

where $\Gamma$, the shed circulation, and $D_{T}$, the turbulent diffusivity, are given by

$$
\Gamma=2 \bar{U} h \tan \alpha
$$

and

$$
D_{T} \sim\left(\frac{1-r}{1+r}\right)^{2} \bar{U} x
$$

respectively[3][5][6], and where $\bar{U}$ is the average inlet velocity, $\rho_{1}$ and $\rho_{2}$ are the unburned and burned gas densities respectively, $\mathrm{t}$ is the time measured from the start of the vortex motion, $\xi$ is the initial radial position of the flame element, and $\xi^{*}$ is the radius of the initial unburned core. It should be noted that $\xi^{*} \sim t^{\frac{1}{2}}$, thus the value of $\beta^{*}$ is independent of time.

A scaling developed for the volumetric entrainment into a planar shear layer by Brown and Roshko[13] is given by 


$$
Q \sim\left(\frac{1-r}{1+r}\right) U_{2} x
$$

where $U_{2}$ is the high speed stream inlet velocity. Comparing Equations 4.9 and 4.10

$$
D_{T} \sim Q^{2}
$$

Substituting this result into Equation 4.5, the scaling for mixing rate augmentation becomes

$$
\frac{d M_{a}}{d(x / \lambda)} \sim \frac{\Gamma^{\frac{2}{3}} Q^{\frac{2}{3}}}{\bar{U} \lambda} \frac{1}{\pi} \int_{0}^{\beta^{*}}\left\{[J(\beta)]^{-\frac{1}{2}}\left(\frac{\left(\frac{Q^{2}}{\Gamma}\right)^{2}+\beta^{2}}{1+\frac{\beta}{\beta^{*}}\left(\frac{\rho_{1}}{\rho_{2}}-1\right)}\right)-\left(\frac{Q^{2}}{\Gamma}\right)^{\frac{1}{3}} \beta^{-\frac{1}{2}}\right\} \frac{d \beta}{\beta}
$$

For each test condition, $\bar{U}$ and $\lambda$ were constant, while $\Gamma$ was constant for each lobed mixer run since it is only a function of geometry. Thus the ratio of any two mixing augmentation rates is expected to scale as

$$
\frac{\left.\frac{d M_{a}}{d(x / \lambda)}\right|_{\Phi_{1}}}{\left.\frac{d M_{a}}{d(x / \lambda)}\right|_{\Phi_{2}}}=\frac{\left.Q^{\frac{2}{3}} \Lambda\right|_{\Phi_{1}}}{\left.Q^{\frac{2}{3}} \Lambda\right|_{\Phi_{2}}}
$$

at a given $\mathrm{x} / \lambda$ where $\Lambda$ is the integral from 0 to $\beta^{*}$ given in Equation 4.12. Evaluation of this scaling law for varying heat release shows that the mixing rate augmentation due to streamwise vorticity is expected to decrease as

$$
\frac{\left.\frac{d M_{a}}{d(x / \lambda)}\right|_{\Phi=1.48}}{\left.\frac{d M_{a}}{d(x / \lambda)}\right|_{\Phi=0}}=0.492
$$

for the low heat release case $(\Phi=1.48)$ and as 


$$
\frac{\left.\frac{d M_{a}}{d(x / \lambda)}\right|_{\Phi=1.84}}{\left.\frac{d M_{a}}{d(x / \lambda)}\right|_{\Phi=0}}=0.304
$$

for the high heat release case $(\Phi=1.84)$. The observed mixing rate augmentation can then be compared to the expected values. The results of this comparison are given in Table 4.5.

Table 4.5: Comparison of expected and observed values of $d M_{a}$.

\begin{tabular}{|c|c|c|}
\hline Heat Release, $\Phi$ & \multicolumn{2}{|c|}{$d M_{a}$} \\
\cline { 2 - 3 } & Expected & Observed \\
\hline 0 & 0.050 & 0.050 \\
\hline 1.48 & 0.025 & 0.041 \\
\hline 1.84 & 0.015 & 0.041 \\
\hline
\end{tabular}

It can been seen that although a $70 \%$ decrease in the augmented mixing rate, $d M_{a}$, was expected, only an $18 \%$ decrease in the augmented mixing rate and a $51 \%$ decrease in the overall mixing rate, $d M_{t o t}$, were observed. The poor agreement between the expected and observed values of augmented mixing rate implies that the Karagozian and Marble model may not be applicable to lobed mixer flows with large amounts of heat release. This could be due to one or more invalid assumptions in the model. In particular, the assumption of an isolated vortex, along with semi-infinite fuel and oxidizer flows, are likely sources of error. The shed vortices merge after approximately 2 lobe wavelengths downstream of the trailing edge, and the depletion of reactants between the vortices could cause a reduction in the heat release generated by a given vortex from the theoretical heat release with a single vortex. This reduction in heat release would cause a smaller reduction in the effective diffusion coefficient than expected, thereby lessening the reduction in mixing rate augmentation due to heat release. 


\section{Chapter 5}

\section{Conclusions}

A lobed mixer and flat plate were tested in both reacting and non-reacting environments. The mixing rates were calculated by comparing experimental static pressure and mass-averaged total enthalpy profiles with the predictions of a quasi-one dimensional control volume model. The control volume model performed well, giving insights into the behavior of state variables and allowing determination of mixing rates with a small computational effort (approximately one minute on an IBM RS6000 workstation).

The conclusions of this study are:

1. Mixing augmentation occurred when streamwise vorticity was added to a reacting flow using a lobed mixer. The initial mixing rate of the lobed mixer was greater than the flat plate mixing rate by a factor of between 6 and 12 for the different heat release cases.

2. The mixing rate in the near field of the lobed mixer was found to be less sensitive to the detrimental effects of heat release than the planar shear layer mixing rate. The planar shear layer mixing rate $\left(d M_{p s l}\right)$ was decreased by $76 \%$ from zero to high heat release, while the overall mixing rate for the lobed mixer was decreased by $51 \%$, with the contribution associated with the streamwise vorticity decreasing by only $18 \%$.

3. The decrease in mixing rate with increasing heat release for a flat plate compared 
well with the results presented by Hermanson and Dimotakis.

4. The mixing rate associated with a lobed mixer was found to decrease with increasing heat release after the streamwise vorticity had decayed. The decay in mixing rate with heat release in the far field was approximately proportional to the decay in mixing rate observed for the flat plate. 


\section{Bibliography}

[1] Boeing Commercial Airplanes, "High-Speed Civil Transport Study," NASA CR4233, September 1989.

[2] McCormick, D. C., "Vortical and Turbulent Structure of Planar and Lobed Mixer Free-Shear Layers," Ph.D. Thesis, Univ. of Conn., 1992.

[3] Fung, A. K. S., "Modeling of Mixer-Ejector Nozzle Flows," S.M. Thesis, Mass. Institute of Tech., 1995.

[4] O'Sullivan, M. N., "A Computational Study of the Effects of Viscosity on Lobed Mixer Flowfields," S.M. Thesis, Mass. Institute of Tech., 1993.

[5] Manning, T. A., "Experimental Study of Mixing Flows with Streamwise Vorticity," S.M. Thesis, Mass. Institute of Tech., 1991.

[6] Qiu, Y. J., "A Study of Streamwise Vortex Enhanced Mixing in Lobed Mixer Devices," Ph.D. Thesis, Mass. Institute of Tech., 1992.

[7] Seiner, J. M., Krejsa, E. A., "Supersonic Jet Noise and the High Speed Civil Transport," Joint Propulsion Conference, Monterey, CA, July 1989, AIA A Paper 89-2358.

[8] McVey, J. B., Peschke, W. T., "Study of Streamwise Vorticity-Stirred Combustion," UTRC Report R93-958160-1, 1993.

[9] Krasnodebski, J., Aero-Environmental Research Lab, Mass. Institute of Tech., Personal Communication, 1995. 
[10] Hermanson, J. C., Dimotakis, P. E., "Effects of Heat Release in a Turbulent, Reacting Shear Layer," Journal of Fluid Mechanics, Vol. 199, 1989, pp. 333-375.

[11] Hermanson, J. C., "Heat Release Effects in a Turbulent, Reacting Shear Layer," Ph.D. Thesis, California Institute of Tech., 1985.

[12] Konrad, J. H., "An Experimental Investigation of Mixing in Two Dimensional Shear Flows with Application to Diffusion Limited Chemical Reactions," Ph.D. Thesis, California Institute of Tech., 1976.

[13] Brown, G. L., Roshko, A., "On Density Effects and Large Structure in Turbulent Mixing Layers," Journal of Fluid Mechanics, Vol. 64, part 4, 1974, pp. 775-816.

[14] Karagozian, A. R., Marble, F. E., "Study of a Diffusion Flame in a Stretched Vortex," Combustion Science and Technology, Vol. 45, 1986, pp. 65-84.

[15] Chedaille, J., Braud, Y., Measurements in Flames, Crane, Russak \& Company, Inc., 1972.

[16] OMEGA Engineering, Inc., OMEGA Complete Temperature Measurement Handbook and Encyclopedia, Vol. 29, 1995. 


\section{Appendix A}

\section{Thermocouple Corrections}

The total temperature measurements made with the thermocouple were corrected for radiation, conduction, and catalytic effects. The thermocouple was type $\mathrm{R}$. This type has one lead of platinum and the other of platinum with $13 \%$ rhodium. Its maximum recommended temperature is $2000 \mathrm{~K}$ which is close to the maximum temperatures measured in this study.

In Section A.1, the radiation corrections are described, followed by the conduction corrections in Section A.2. The correction due to catalytic effects are presented in Section A.3. The corrections for radiation and conduction were taken from Chedaille and Braud[15], while the catalytic corrections were taken from the OMEGA temperature $\operatorname{book}[16]$.

\section{A.1 Radiation Correction}

The heat exchange due to radiation between the thermocouple and the walls of the tunnel and the gas is given by $\sigma T^{4}$ where $\sigma$ is the Stefan-Boltzman constant. The heat exchange coefficient is then given by

$$
\Phi_{r}=\epsilon \sigma\left(T_{j}^{4}-T_{\text {surr }}^{4}\right)
$$


where $T_{j}$ is the thermocouple junction temperature, $T_{\text {surr }}$ is the temperature of the surrounding gas or walls, and $\epsilon$ is the emissivity of the thermocouple.

Assuming that the gas was transparent, the surroundings were determined to be at a temperature of $478 \mathrm{~K}$. The walls were made of 304 stainless steel and the windows were made of quartz. The error can be expressed as

$$
\Delta T=\left(T_{j}-T_{\text {surr }}\right) \frac{h_{r}}{h_{c}}
$$

where

$$
h_{r}=4 \epsilon \sigma T_{\text {surr }}^{3}
$$

is the the radiation transfer coefficient, and

$$
h_{c}=\frac{N u K_{g}}{D}
$$

is the convection transfer coefficient, and where $K_{g}$ is the thermal conductivity of air, $\mathrm{Nu}$ is the Nusselt number, and $\mathrm{D}$ is the junction diameter. An average emissivity of 0.6 was used for the quartz $/ 304$ stainless walls. For these experiments, $D=0.035$ in, $K_{g}=40 \times 10^{-3} \mathrm{~W} /(m \cdot K)$, and $\sigma=5.67 \times 10^{-8} \mathrm{~W} /\left(m^{2} \cdot K^{4}\right)$. For a thermocouple normal to the flow direction

$$
N u=.44\left(R e_{D}\right)^{\frac{1}{2}}
$$

where $R e_{D}$ is the Reynolds number based on the junction diameter. Using $R e_{D}=3350$, the Nusselt number is then $\mathrm{Nu}=25.5$. Plugging these numbers into Equations $\mathrm{A} .3$ and A.4, the convection and radiation coefficients were estimated at $h_{r}=1150 \mathrm{~W} /\left(\mathrm{m}^{2} \cdot K\right)$ 
and $h_{c}=15 \mathrm{~W} /\left(m^{2} \cdot K\right)$.

This analysis yielded the relation

$$
T_{g}=1.013 T_{j}-6 K
$$

where $T_{g}$ is the true gas temperature.

\section{A.2 Conduction Correction}

The error in total temperature is given by

$$
\Delta T=\frac{T_{j}-T_{b a s e}}{L\left(4 h_{c} / D \lambda\right)^{\frac{1}{2}}}
$$

where $T_{b a s e}$ is the temperature of the thermocouple base, $\mathrm{L}$ is the length of wire between the junction and base, $\mathrm{D}$ is the diameter of the leads, and $\lambda$ is the thermal conductivity of the thermocouple. For these experiments, $T_{b a s e}=.95 T_{j}, \mathrm{~L}=.19 \mathrm{in}$, $\mathrm{D}=0.020$ in, $\lambda=73 \mathrm{~W} /(m \cdot K)$, and $h_{c}=15 \mathrm{~W} /\left(m^{2} \cdot K\right)$ as in Section A.1.

The result of this analysis was the relation

$$
T_{g}=1.02 T_{j}
$$

where $T_{g}$ is again the true gas temperature.

\section{A.3 Catalytic Correction}

Platinum is a catalyst for reactions in an oxidizing environment. This means that some reactions occurred at the surface of the thermocouple which raised the measured temperature slightly. These effects were estimated through consideration of the 
temperature, pressure, and surface area of the thermocouple.

These effects are correlated in the OMEGA temperature book. The correlation estimated the error in temperature as $2 \mathrm{~K}$. This yielded the relation

$$
T_{g}=T_{j}-2 K
$$

where $T_{g}$ is the gas temperature and $T_{j}$ is the junction temperature. 


\section{Appendix B}

\section{Sensitivity Study}

The sensitivity of mixing rate to the changes in the static pressure and mass averaged total enthalpy profiles shown in Figures 4-10 and 4-11 was evaluated. The procedure was to change the mixing rate until the static pressure profile computed by the control volume model passed through the high points of the experimental error bands and also through the low points. This was repeated for the total enthalpy profiles. The criteria used to determine when the static pressure and total enthalpy profiles passed through the high and low points was that the error in static pressure be no greater than $5 \%$ at four of the five measurement points and that the error in total enthalpy be no greater than $5 \%$ at three of the four measurement points. The results for the flat plate are presented in Section B.1 followed by the results for the lobed mixer in Section B.2.

\section{B.1 Flat Plate}

Figure B-1 shows the static pressure profiles matched to the high and low points of the error bars for the zero heat release case. The solid line represents an over estimation of the mixing rate, while the dashed line represents an under estimation.

The maximum and minimum mixing rates corresponding to the maximum and minimum static pressure profiles in Figure B-1 are shown in Figure B-2 as the dashed lines. The solid line represents the best match for the static pressure. The dashed 


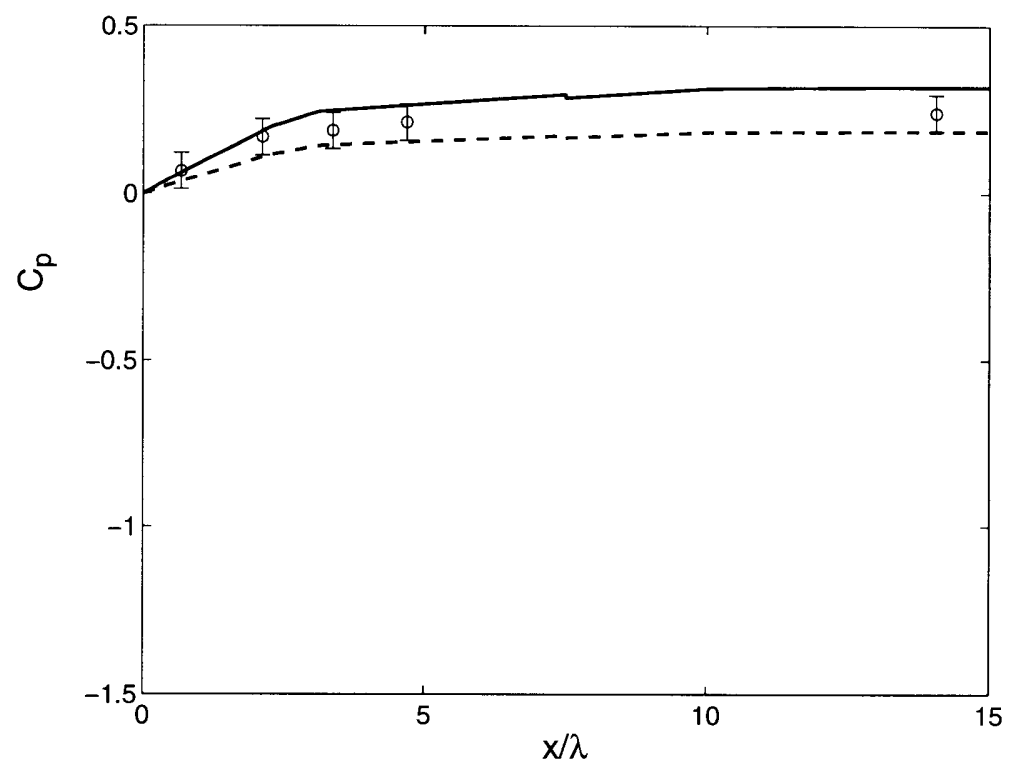

Figure B-1: Maximum (solid) and minimum (dashed) static pressure profiles for the flat plate with zero heat release $(\Phi=0)$.

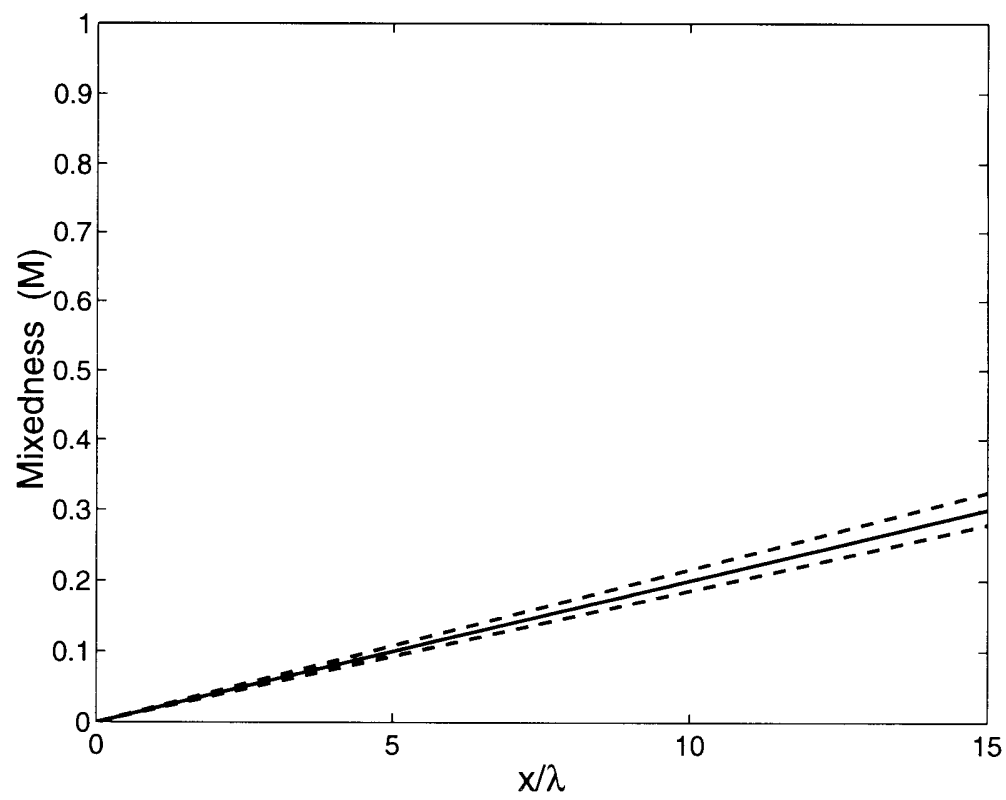

Figure B-2: Maximum and minimum mixing rates (dashed) and best mixing rate (solid) for the flat plate with zero heat release $(\Phi=0)$. 


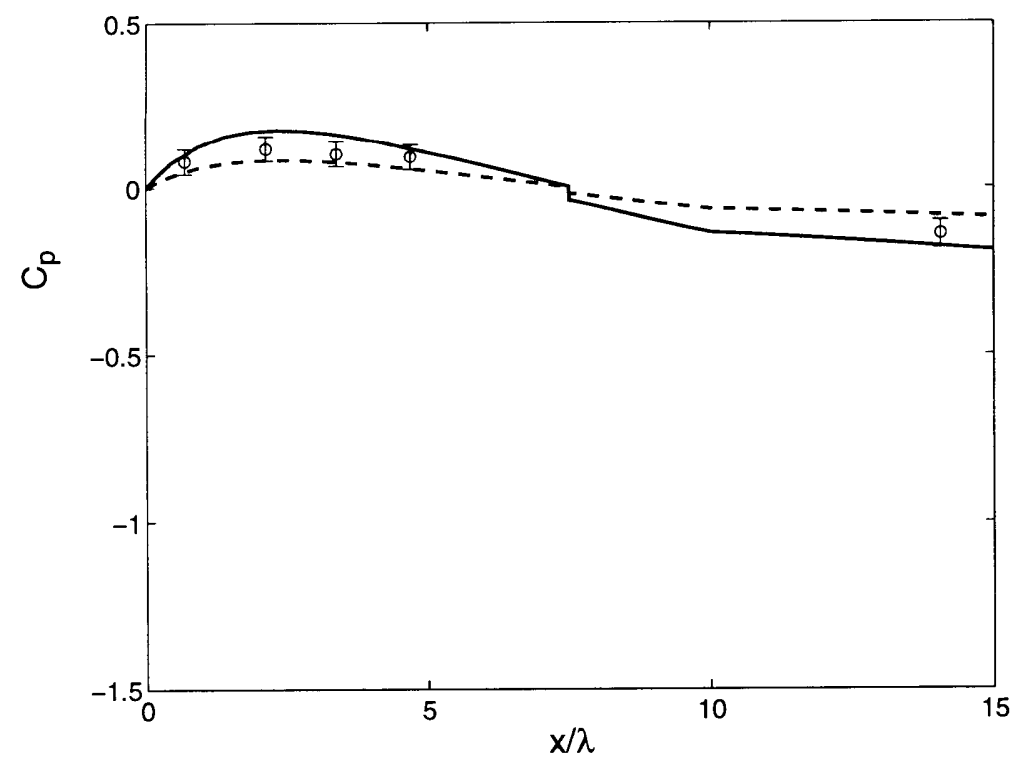

Figure B-3: Maximum (solid) and minimum (dashed) static pressure profiles for the flat plate with low heat release $(\Phi=1.48)$.

lines are the bounds on the shaded error bands seen in Figure 4-12. Figure B-2 shows that the mixing rate changed about $\pm 8 \%$ when the static pressure was matched high and low.

Figure B-3 shows the high and low static pressure profiles for the flat plate with low heat release. The mass averaged total enthalpy profiles for the minimum and maximum mixing rates are shown in Figure B-4. The mixing rates corresponding to these profiles are shown in Figure B-5 along with the best mixing rate. It can be seen that the mixing rates used to compute the total enthalpy profiles, shown as dotted lines, deviated less from the best mixing rate than the mixing rates used to compute the static pressure profiles. This implies that static pressure was a more sensitive measure of mixing rate than total enthalpy. For this reason, the mixing rate in the control volume model was changed until the experimental and computed static pressure profiles were matched in the final determination of the mixing rate for each test condition. The total enthalpy profiles were then checked to confirm that they were also matched. For every case, when the static pressure profiles were matched, the mass averaged total enthalpy profiles were also matched. As a result, the bounds on the shaded error bands in Figure 4-12 are the dashed lines corresponding to the 


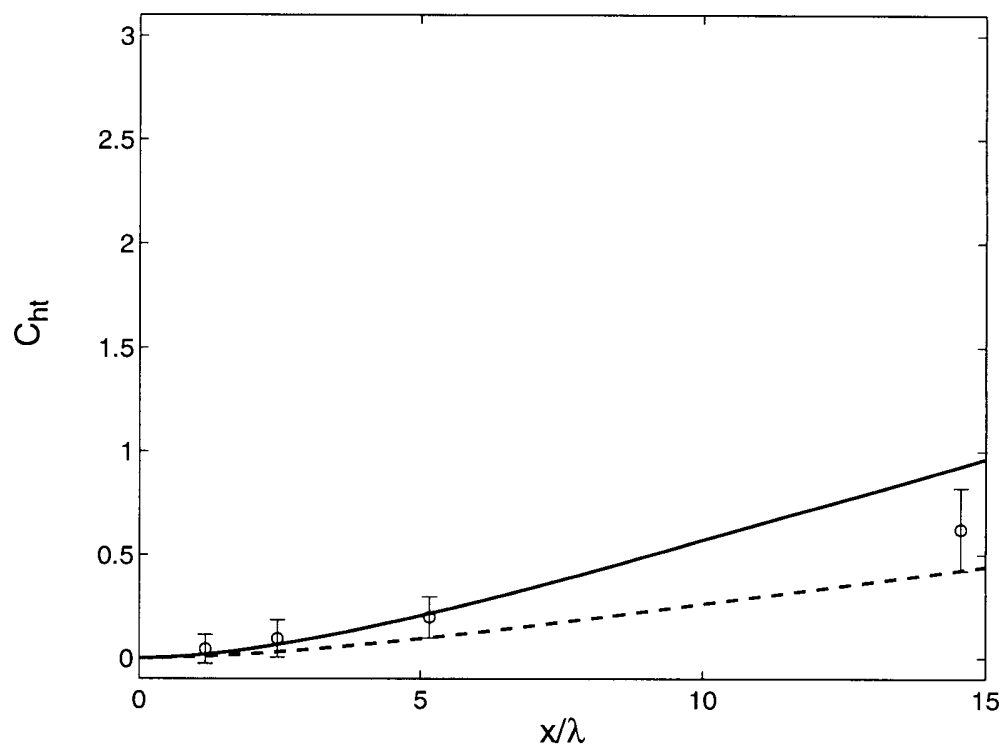

Figure B-4: Maximum (solid) and minimum (dashed) mass averaged total enthalpy profiles for the flat plate with low heat release $(\Phi=1.48)$.

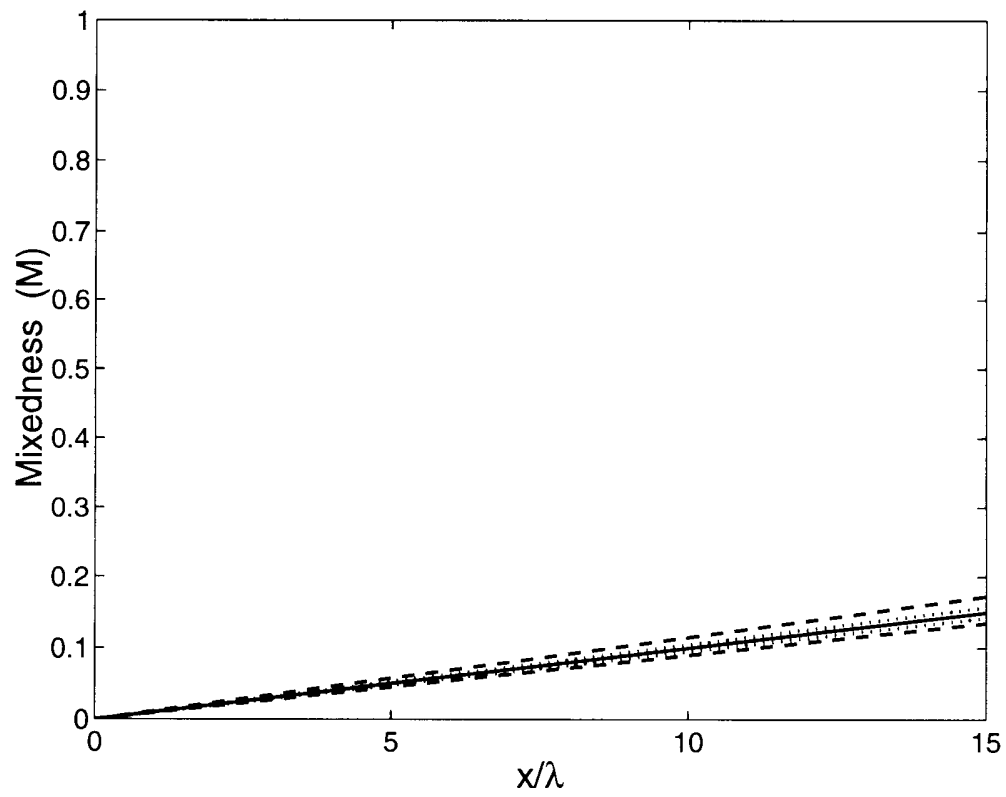

Figure B-5: Maximum and minimum mixing rates associated with the static pressure profiles (dashed) and the total enthalpy profiles (dotted) along with the best mixing rate (solid) for the flat plate with low heat release $(\Phi=1.48)$. 


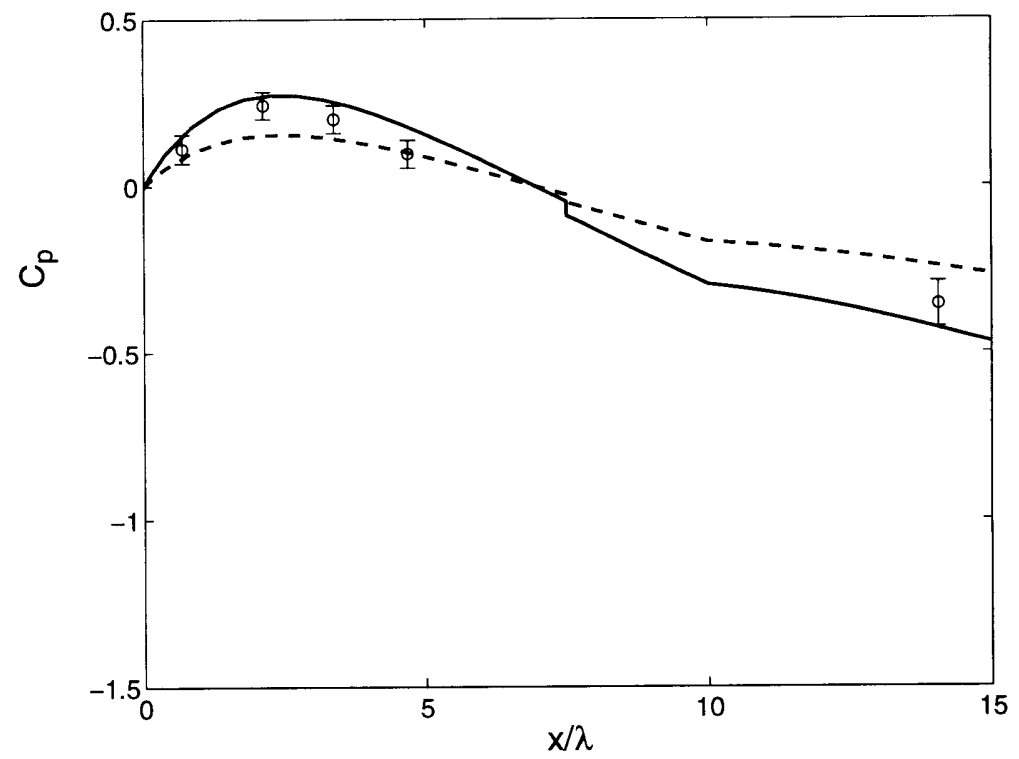

Figure B-6: Maximum (solid) and minimum (dashed) static pressure profiles for the flat plate with high heat release $(\Phi=1.84)$.

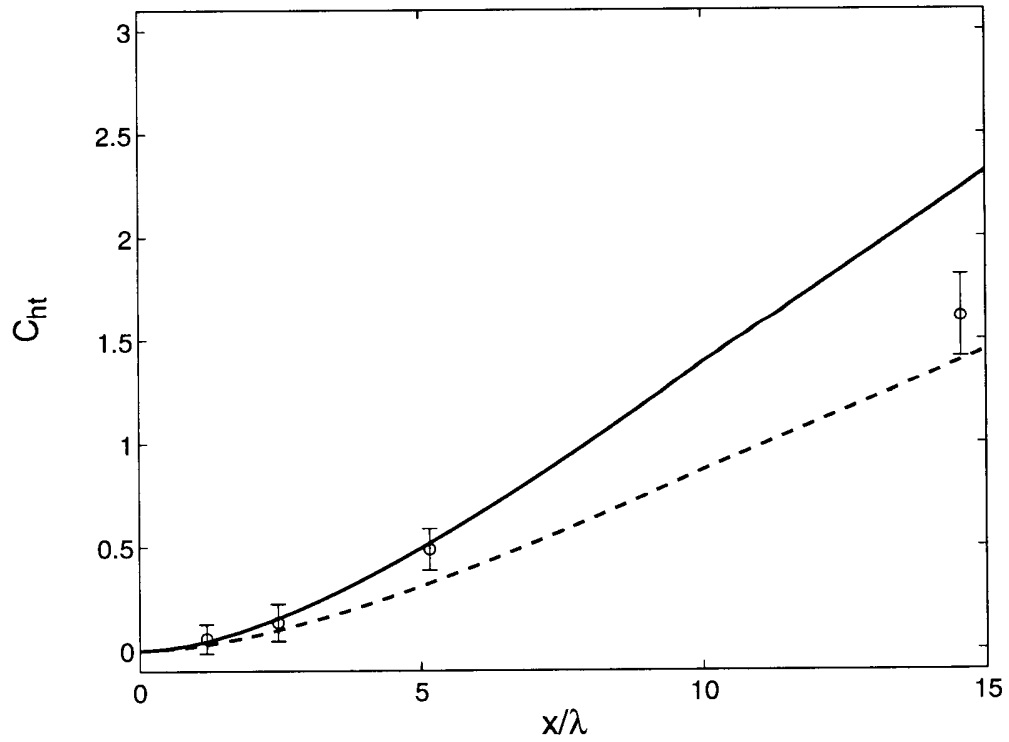

Figure B-7: Maximum (solid) and minimum (dashed) mass averaged total enthalpy profiles for the flat plate with high heat release $(\Phi=1.84)$. 


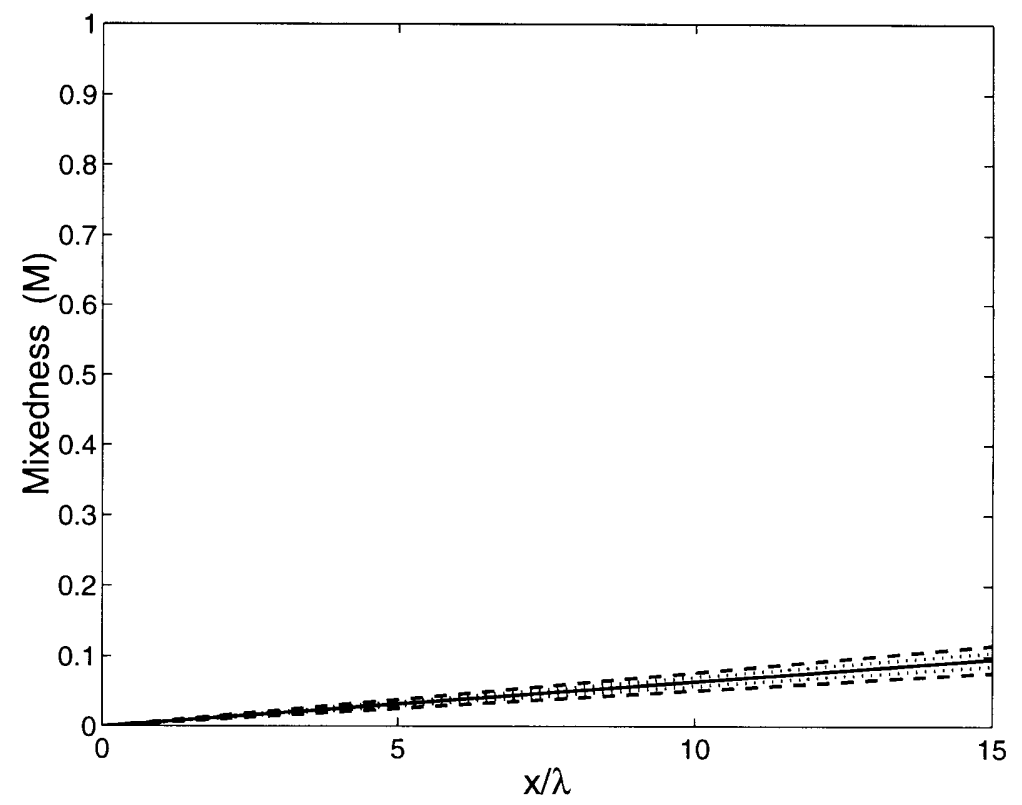

Figure B-8: Maximum and minimum mixing rates associated with the static pressure profiles (dashed) and the total enthalpy profiles (dotted) along with the best mixing rate (solid) for the flat plate with high heat release $(\Phi=1.84)$.

mixing rates used to compute the high and low static pressure profiles on all of the mixing rate plots in this appendix.

Figure B-6 shows the high and low static pressure profiles for the flat plate with high heat release. The mass averaged total enthalpy profiles for the minimum and maximum mixing rates are shown in Figure B-7. The mixing rates corresponding to these profiles are shown in Figure B- 8 along with the best mixing rate.

\section{B.2 Lobed Mixer}

Figure B-9 shows the static pressure profiles matched to the high and low points of the error bars for the zero heat release case. The solid line represents an over estimation of the mixing rate, while the dashed line represents an under estimation.

The maximum and minimum mixing rates corresponding to the maximum and minimum static pressure profiles in Figure B-9 are shown in Figure B-10 as the dashed lines. The solid line represents the best match for the static pressure.

Figure B-11 shows the high and low static pressure profiles for the lobed mixer 


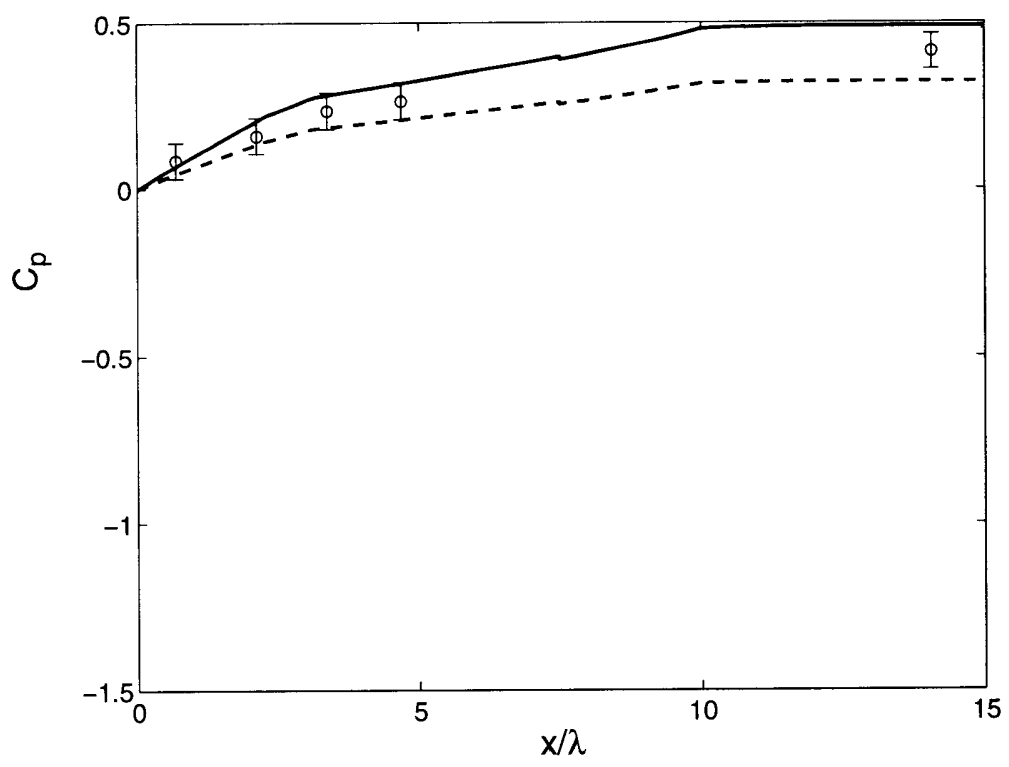

Figure B-9: Maximum (solid) and minimum (dashed) static pressure profiles for the lobed mixer with zero heat release $(\Phi=0)$.

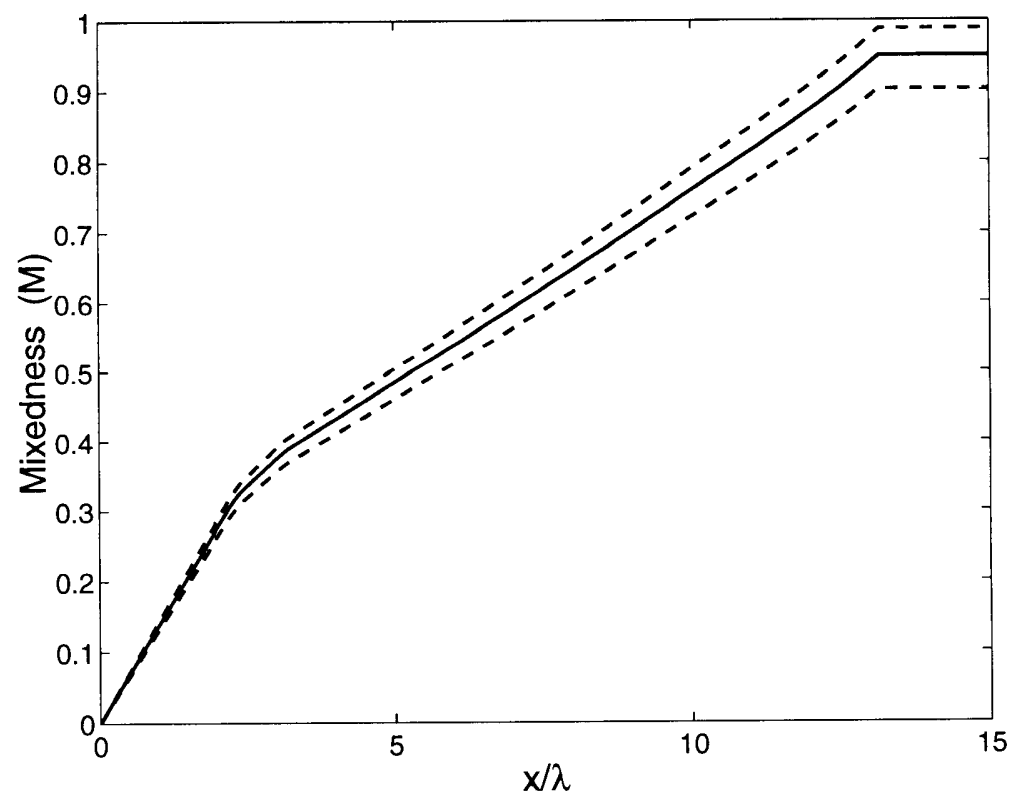

Figure B-10: Maximum and minimum mixing rates (dashed) and best mixing rate (solid) for the lobed mixer with zero heat release $(\Phi=0)$. 


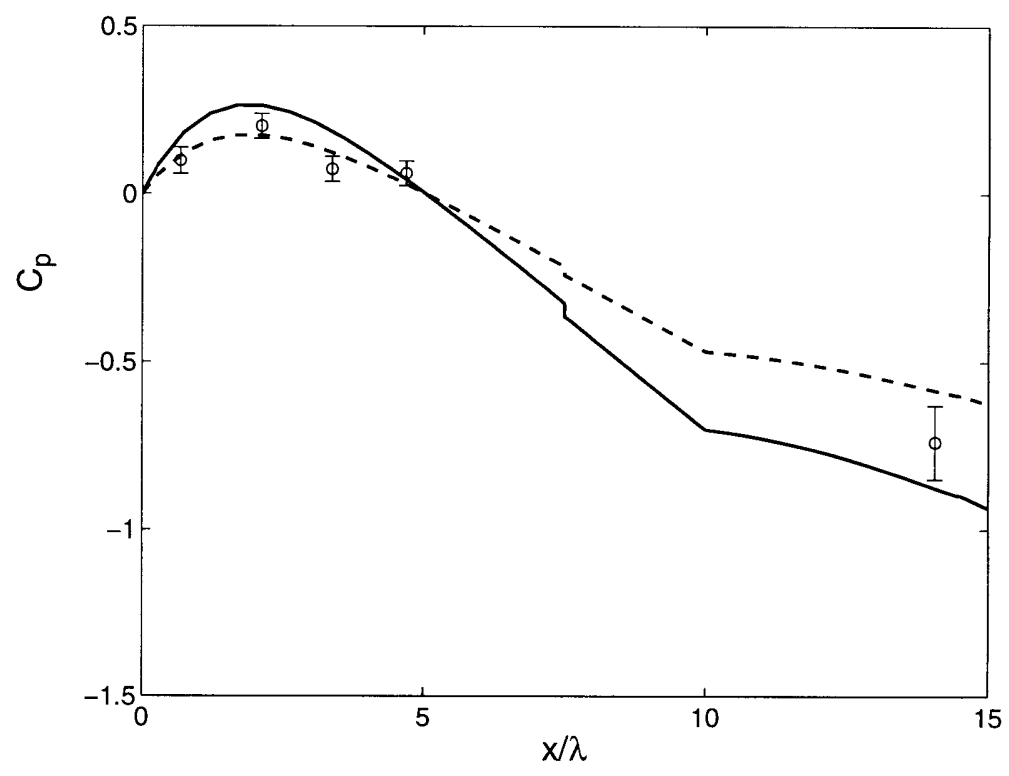

Figure B-11: Maximum (solid) and minimum (dashed) static pressure profiles for the lobed mixer with low heat release $(\Phi=1.48)$.

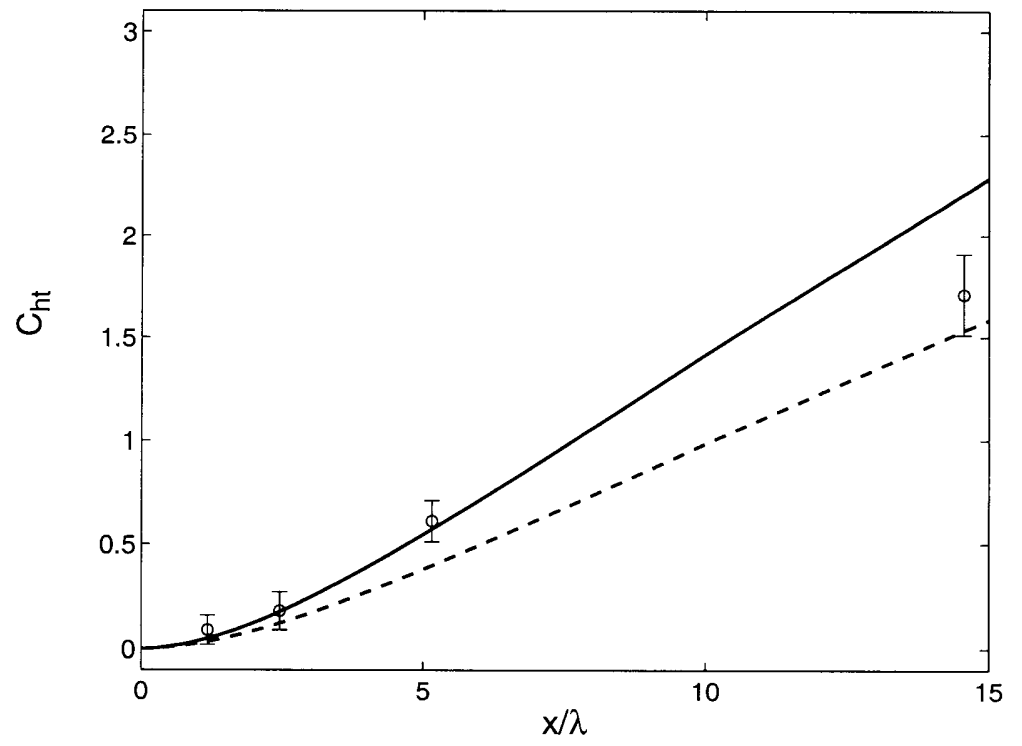

Figure B-12: Maximum (solid) and minimum (dashed) mass averaged total enthalpy profiles for the lobed mixer with low heat release $(\Phi=1.48)$. 


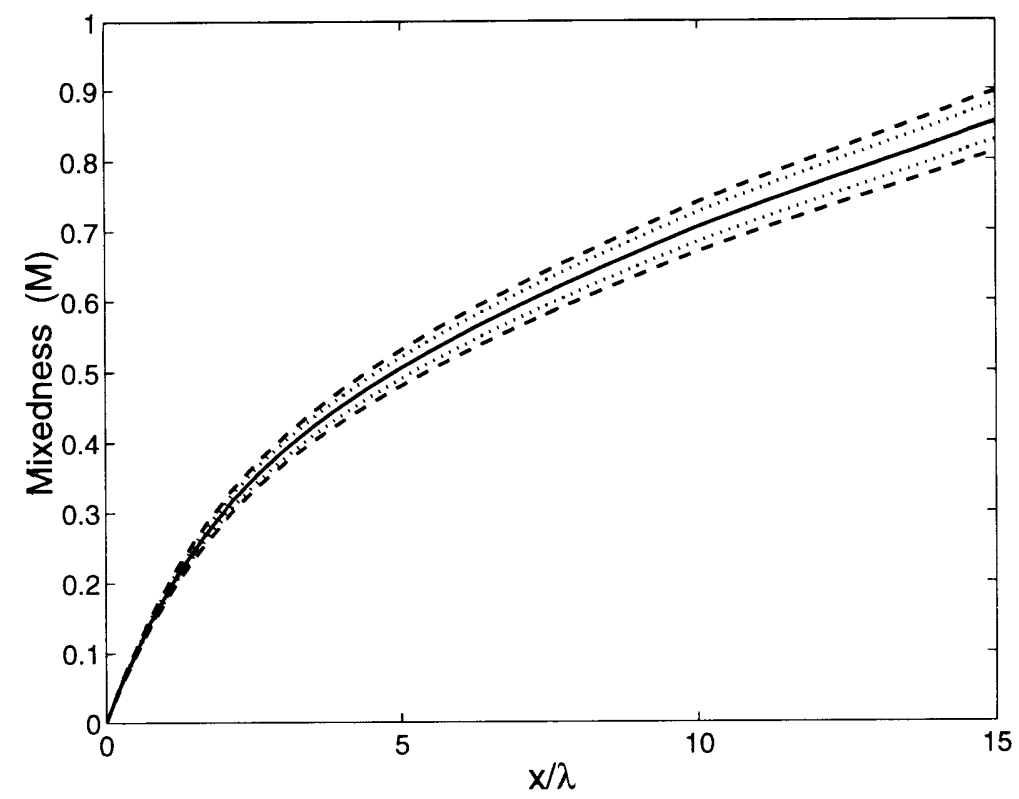

Figure B-13: Maximum and minimum mixing rates associated with the static pressure profiles (dashed) and the total enthalpy profiles (dotted) along with the best mixing rate (solid) for the lobed mixer with low heat release $(\Phi=1.48)$.

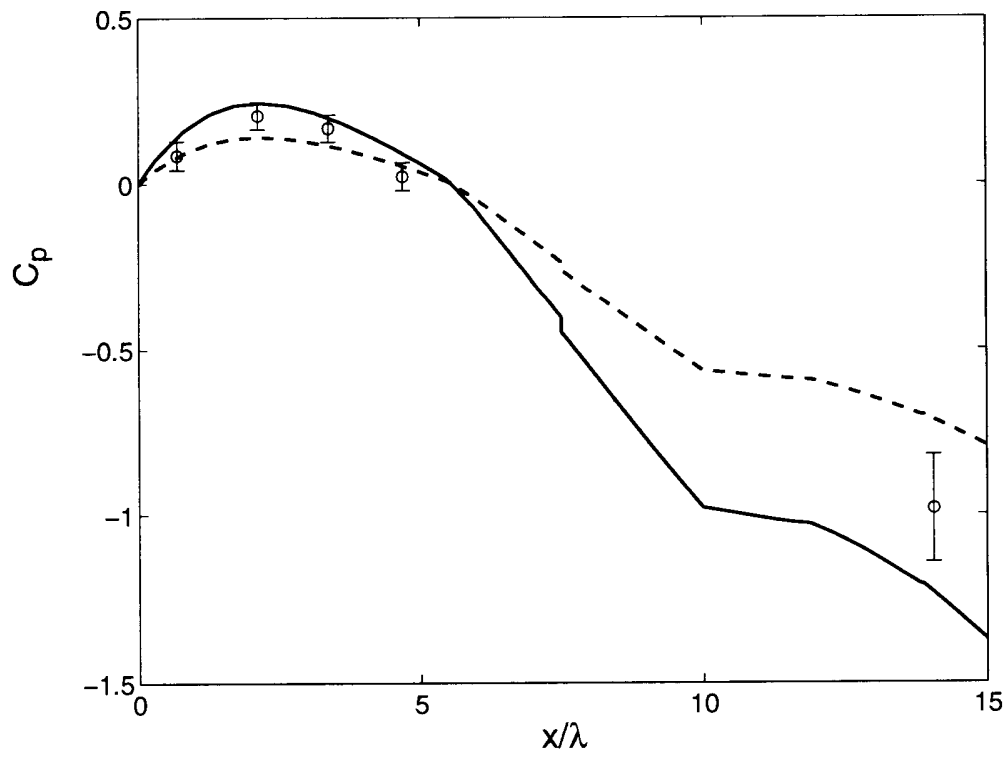

Figure B-14: Maximum (solid) and minimum (dashed) static pressure profiles for the lobed mixer with high heat release $(\Phi=1.84)$. 


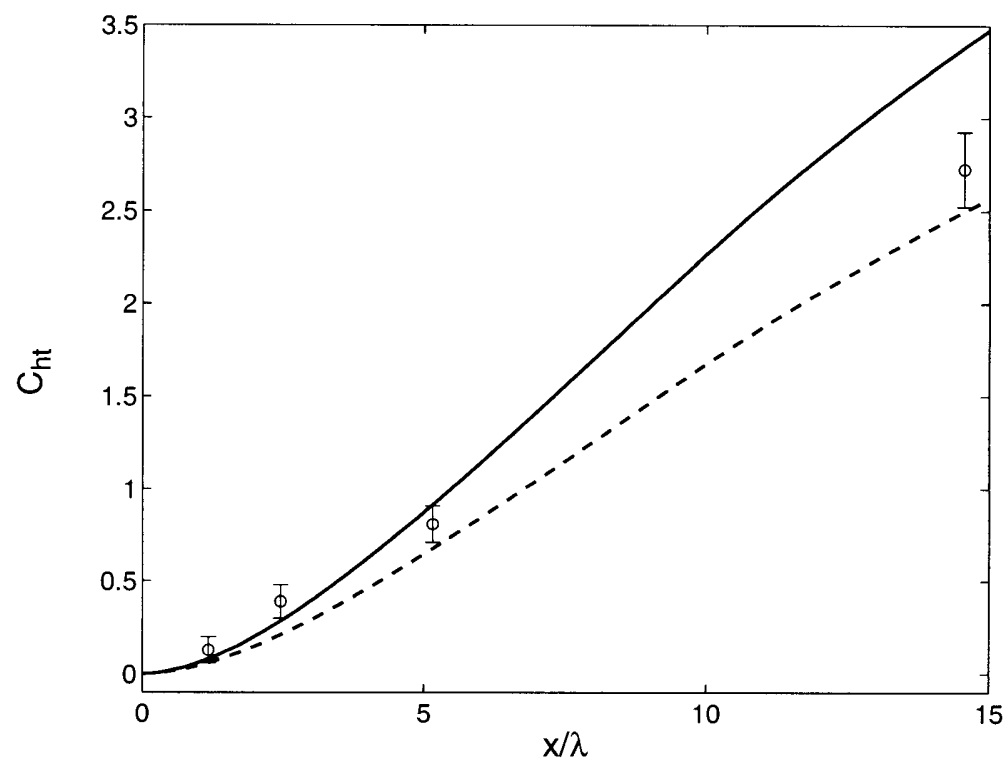

Figure B-15: Maximum (solid) and minimum (dashed) mass averaged total enthalpy profiles for the lobed mixer with high heat release $(\Phi=1.84)$.

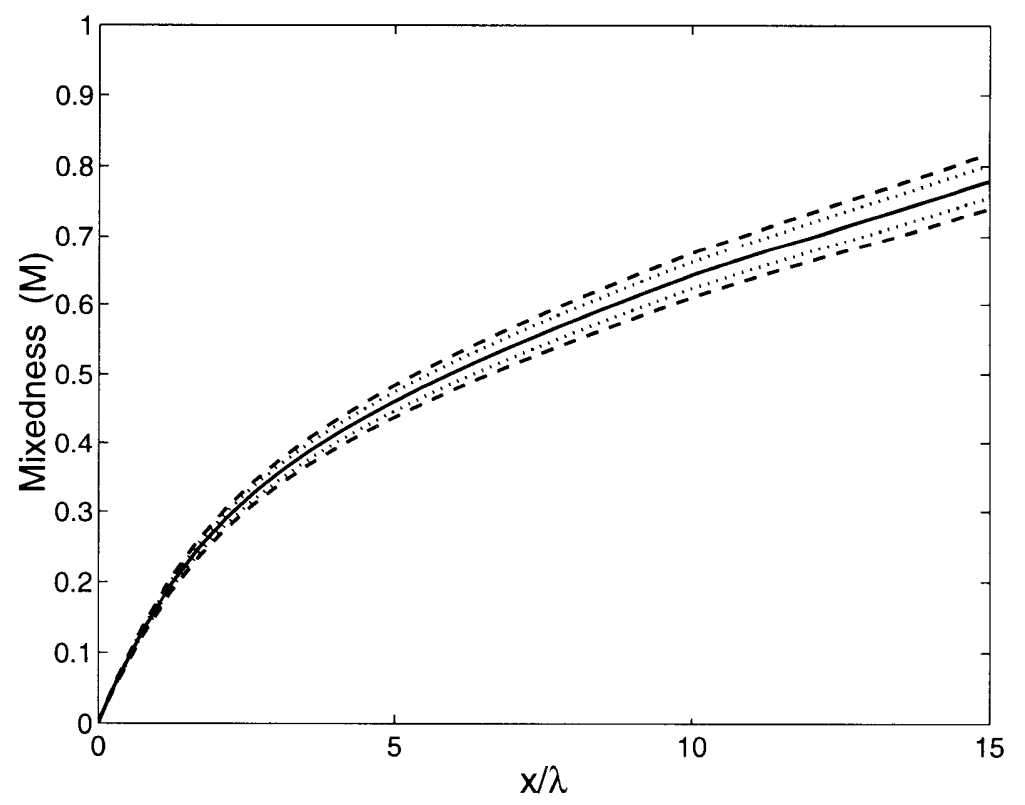

Figure B-16: Maximum and minimum mixing rates associated with the static pressure profiles (dashed) and the total enthalpy profiles (dotted) along with the best mixing rate (solid) for the lobed mixer with high heat release $(\Phi=1.84)$. 
with low heat release. The mass averaged total enthalpy profiles for the minimum and maximum mixing rates are shown in Figure B-12. The mixing rates corresponding to these profiles are shown in Figure B-13 along with the best mixing rate.

Figure B-14 shows the high and low static pressure profiles for the lobed mixer with high heat release. The mass averaged total enthalpy profiles for the minimum and maximum mixing rates are shown in Figure B-15. The mixing rates corresponding to these profiles are shown in Figure B-16 along with the best mixing rate. 


\section{Appendix C}

\section{Control Volume Model}

The details of the control volume model described in Section 3.2 are presented in this appendix. First, the control volume equations are presented in Section C.1, followed by the model for the entrainment of fluid into the various shear layers in Section C.2. Finally, the combustion chemistry is discussed in Section C.3.

\section{C.1 Control Volume Equations}

The duct was broken into three computational sections. The first ran from the trailing edge of the mixer to the step change in area at $x / \lambda=7.5$ due to the film cooling housings shown in Figure 3-3. The second section spanned the distance $7.5 \leq x / \lambda<10$ from the start of the film cooling housing to its end. The third section ran from the end of the film cooling housing at $x / \lambda=10$ to the end of the computational domain at $\mathrm{x} / \lambda=15$. The actual test section extended to $\mathrm{x} / \lambda=25$, but there were no measurements made past $\mathrm{x} / \lambda=14.6$. Each stream was defined by the three conservation equations (mass, momentum, and energy) plus an equation of state. These equations were integrated using a fourth order Runge-Kutta method on an IBM RS6000.

\section{C.1.1 Sections 1 and 2}

Stream 1 is defined by 


$$
\begin{aligned}
& \rho_{1} u_{1} \partial A_{1}+\rho_{1} A_{1} \partial u_{1}+u_{1} A_{1} \partial \rho_{1}-\partial \dot{m}_{1}=0 \\
& A_{1} \partial p+\rho_{1} u_{1} A_{1} \partial u_{1}+\left(u_{1}-u_{2}\right) \partial \dot{m}_{2,1}+\left(u_{1}-u_{3}\right) \partial \dot{m}_{3,1}=0 \\
& u_{1} \partial u_{1}+C_{p_{1}} \partial T_{1}+\frac{\left\{C_{p_{2}}\left(T_{1}-T_{2}\right)+\frac{1}{2} u_{1}^{2}-\frac{1}{2} u_{2}^{2}\right\} \partial \dot{m}_{2,1}}{\dot{m}_{1}} \\
&+\frac{\left\{C_{p_{3}}\left(T_{1}-T_{3}\right)+\frac{1}{2} u_{1}^{2}-\frac{1}{2} u_{3}^{2}\right\} \partial \dot{m}_{3,1}}{\dot{m}_{1}}-\partial h_{p r_{1}}=0 \\
& \frac{1}{p} \partial p-\frac{1}{T_{1}} \partial T_{1}-\frac{1}{\rho_{1}} \partial \rho_{1}=0
\end{aligned}
$$

where $\dot{m}_{1}$ is the total mass flow in stream $1, \partial \dot{m}_{1,2}$ and $\partial \dot{m}_{1,3}$ are the mass flows entrained into stream 1 from streams 2 and 3 respectively, and $\partial h_{p r_{1}}$ is the change in enthalpy due to the conversion of reactants into products in stream 1.

Stream 2 is defined by

$$
\begin{aligned}
\rho_{2} u_{2} \partial A_{2}+\rho_{2} A_{2} \partial u_{2}+u_{2} A_{2} \partial \rho_{2}+\partial \dot{m}_{2,1}+\partial \dot{m}_{2,4} & =0 \\
A_{2} \partial p+\rho_{2} u_{2} A_{2} \partial u_{2}-u_{2} \partial \dot{m}_{2,1}-u_{2} \partial \dot{m}_{2,4} & =0 \\
u_{2} \partial u_{2}+C_{p_{2}} \partial T_{2} & =0 \\
\frac{1}{p} \partial p-\frac{1}{T_{2}} \partial T_{2}-\frac{1}{\rho_{2}} \partial \rho_{2} & =0
\end{aligned}
$$

where $\partial \dot{m}_{2,1}$ and $\partial \dot{m}_{2,4}$ are the mass flows entrained from stream 2 into streams 1 and 4 respectively.

Stream 3 is defined by

$$
\begin{aligned}
\rho_{3} u_{3} \partial A_{3}+\rho_{3} A_{3} \partial u_{3}+u_{3} A_{3} \partial \rho_{3}+\partial \dot{m}_{3,1}+\partial \dot{m}_{3,5}=0 \\
A_{3} \partial p+\rho_{3} u_{3} A_{3} \partial u_{3}-u_{3} \partial \dot{m}_{3,1}-u_{3} \partial \dot{m}_{3,5}=0 \\
u_{3} \partial u_{3}+C_{p_{3}} \partial T_{3}=0 \\
\frac{1}{p} \partial p-\frac{1}{T_{3}} \partial T_{3}-\frac{1}{\rho_{3}} \partial \rho_{3}=0
\end{aligned}
$$


where $\partial \dot{m}_{3,1}$ and $\partial \dot{m}_{3,5}$ are the mass flows entrained from stream 3 into streams 1 and 5 respectively.

Stream 4 is defined by

$$
\begin{aligned}
\rho_{4} u_{4} \partial A_{4}+\rho_{4} A_{4} \partial u_{4}+u_{4} A_{4} \partial \rho_{4}-\partial \dot{m}_{4}=0 \\
A_{4} \partial p+\rho_{4} u_{4} A_{4} \partial u_{4}+\left(u_{4}-u_{2}\right) \partial \dot{m}_{2,4}+\left(u_{4}-u_{6}\right) \partial \dot{m}_{6}=0 \\
u_{4} \partial u_{4}+C_{p_{4}} \partial T_{4}+\frac{\left\{C_{p_{2}}\left(T_{4}-T_{2}\right)+\frac{1}{2} u_{4}^{2}-\frac{1}{2} u_{2}^{2}\right\} \partial \dot{m}_{2,4}}{\dot{m}_{4}} \\
+\frac{\left\{C_{p_{6}}\left(T_{4}-T_{6}\right)+\frac{1}{2} u_{4}^{2}-\frac{1}{2} u_{6}^{2}\right\} \partial \dot{m}_{6}}{\dot{m}_{4}}=0 \\
\frac{1}{p} \partial p-\frac{1}{T_{4}} \partial T_{4}-\frac{1}{\rho_{4}} \partial \rho_{4}=0
\end{aligned}
$$

where $\dot{m}_{4}$ is the total mass flow in stream 4 , and $\partial \dot{m}_{2,4}$ and $\partial \dot{m}_{6}$ are the mass flows entrained into stream 4 from streams 2 and 6 respectively.

Stream 5 is defined by

$$
\begin{aligned}
& \rho_{5} u_{5} \partial A_{5}+\rho_{5} A_{5} \partial u_{5}+u_{5} A_{5} \partial \rho_{5}-\partial \dot{m}_{5}=0 \\
& A_{5} \partial p+\rho_{5} u_{5} A_{5} \partial u_{5}+\left(u_{5}-u_{3}\right) \partial \dot{m}_{3,5}+\left(u_{5}-u_{7}\right) \partial \dot{m}_{7}=0 \\
& u_{5} \partial u_{5}+C_{p_{5}} \partial T_{5}+\frac{\left\{C_{p_{3}}\left(T_{5}-T_{3}\right)+\frac{1}{2} u_{5}^{2}-\frac{1}{2} u_{3}^{2}\right\} \partial \dot{m}_{3,5}}{\dot{m}_{5}} \\
&+\frac{\left\{C_{p_{7}}\left(T_{5}-T_{7}\right)+\frac{1}{2} u_{5}^{2}-\frac{1}{2} u_{7}^{2}\right\} \partial \dot{m}_{7}}{\dot{m}_{5}}-\partial h_{p r_{5}}=0 \\
& \frac{1}{p} \partial p-\frac{1}{T_{5}} \partial T_{5}-\frac{1}{\rho_{5}} \partial \rho_{5}=0
\end{aligned}
$$

where $\dot{m}_{5}$ is the total mass flow in stream $5, \partial \dot{m}_{3,5}$ and $\partial \dot{m}_{7}$ are the mass flows entrained into stream 5 from streams 3 and 7 respectively, and $\partial h_{p r_{5}}$ is the change in enthalpy due to the conversion of reactants into products in stream 5 .

Stream 6 is defined by 


$$
\begin{aligned}
\rho_{6} u_{6} \partial A_{6}+\rho_{6} A_{6} \partial u_{6}+u_{6} A_{6} \partial \rho_{6}+\partial \dot{m}_{6} & =0 \\
A_{6} \partial p+\rho_{6} u_{6} A_{6} \partial u_{6}-u_{6} \partial \dot{m}_{6} & =0 \\
u_{6} \partial u_{6}+C_{p_{6}} \partial T_{6} & =0 \\
\frac{1}{p} \partial p-\frac{1}{T_{6}} \partial T_{6}-\frac{1}{\rho_{6}} \partial \rho_{6} & =0
\end{aligned}
$$

where $\partial \dot{m}_{6}$ is the mass flow entrained from stream 6 into stream 4 .

Stream 7 is defined by

$$
\begin{array}{r}
\rho_{7} u_{7} \partial A_{7}+\rho_{7} A_{7} \partial u_{7}+u_{7} A_{7} \partial \rho_{7}+\partial \dot{m}_{7}=0 \\
A_{7} \partial p+\rho_{7} u_{7} A_{7} \partial u_{7}-u_{7} \partial \dot{m}_{7}=0 \\
u_{7} \partial u_{7}+C_{p_{7}} \partial T_{7}=0 \\
\frac{1}{p} \partial p-\frac{1}{T_{7}} \partial T_{7}-\frac{1}{\rho_{7}} \partial \rho_{7}=0
\end{array}
$$

where $\partial \dot{m}_{7}$ is the mass flow entrained from stream 7 into stream 5 .

The final equation is the sum of areas

$$
\partial A_{1}+\partial A_{2}+\partial A_{3}+\partial A_{4}+\partial A_{5}+\partial A_{6}+\partial A_{7}-\partial A=0
$$

where $\partial A$ is the total duct area.

These equations hold for the first two sections of the duct spanning the region $0 \leq x / \lambda<10$.

\section{C.1.2 Section 3}

Stream 1 is defined by 


$$
\begin{aligned}
\rho_{1} u_{1} \partial A_{1}+\rho_{1} A_{1} \partial u_{1}+u_{1} A_{1} \partial \rho_{1}-\partial \dot{m}_{1} & =0 \\
A_{1} \partial p+\rho_{1} u_{1} A_{1} \partial u_{1}+\left(u_{1}-u_{2}\right) \partial \dot{m}_{2,1}+\left(u_{1}-u_{3}\right) \partial \dot{m}_{3,1} & =0 \\
u_{1} \partial u_{1}+C_{p_{1}} \partial T_{1}+\frac{\left\{C_{p_{2}}\left(T_{1}-T_{2}\right)+\frac{1}{2} u_{1}^{2}-\frac{1}{2} u_{2}^{2}\right\} \partial \dot{m}_{2,1}}{\dot{m}_{1}} & \\
+\frac{\left\{C_{p_{3}}\left(T_{1}-T_{3}\right)+\frac{1}{2} u_{1}^{2}-\frac{1}{2} u_{3}^{2}\right\} \partial \dot{m}_{3,1}}{\dot{m}_{1}}-\partial h_{p r_{1}} & =0 \\
\frac{1}{p} \partial p-\frac{1}{T_{1}} \partial T_{1}-\frac{1}{\rho_{1}} \partial \rho_{1} & =0
\end{aligned}
$$

Stream 2 is defined by

$$
\begin{aligned}
\rho_{2} u_{2} \partial A_{2}+\rho_{2} A_{2} \partial u_{2}+u_{2} A_{2} \partial \rho_{2}+\partial \dot{m}_{2,1}+\partial \dot{m}_{2,10} & =0 \\
A_{2} \partial p+\rho_{2} u_{2} A_{2} \partial u_{2}-u_{2} \partial \dot{m}_{2,1}-u_{2} \partial \dot{m}_{2,10} & =0 \\
u_{2} \partial u_{2}+C_{p_{2}} \partial T_{2} & =0 \\
\frac{1}{p} \partial p-\frac{1}{T_{2}} \partial T_{2}-\frac{1}{\rho_{2}} \partial \rho_{2} & =0
\end{aligned}
$$

where $\partial \dot{m}_{2,10}$ is the mass flow entrained from stream 2 into stream 10 .

Stream 3 is defined by

$$
\begin{aligned}
\rho_{3} u_{3} \partial A_{3}+\rho_{3} A_{3} \partial u_{3}+u_{3} A_{3} \partial \rho_{3}+\partial \dot{m}_{3,1}+\partial \dot{m}_{3,11}=0 \\
A_{3} \partial p+\rho_{3} u_{3} A_{3} \partial u_{3}-u_{3} \partial \dot{m}_{3,1}-u_{3} \partial \dot{m}_{3,11}=0 \\
u_{3} \partial u_{3}+C_{p_{3}} \partial T_{3}=0 \\
\frac{1}{p} \partial p-\frac{1}{T_{3}} \partial T_{3}-\frac{1}{\rho_{3}} \partial \rho_{3}=0
\end{aligned}
$$

where $\partial \dot{m}_{3,11}$ is the mass flow entrained from stream 3 into stream 11 .

Stream 4 is defined by 


$$
\begin{aligned}
\rho_{4} u_{4} \partial A_{4}+\rho_{4} A_{4} \partial u_{4}+u_{4} A_{4} \partial \rho_{4}=0 \\
A_{4} \partial p+\rho_{4} u_{4} A_{4} \partial u_{4}=0 \\
u_{4} \partial u_{4}+C_{p_{4}} \partial T_{4}=0 \\
\frac{1}{p} \partial p-\frac{1}{T_{4}} \partial T_{4}-\frac{1}{\rho_{4}} \partial \rho_{4}=0
\end{aligned}
$$

Stream 5 is defined by

$$
\begin{aligned}
\rho_{5} u_{5} \partial A_{5}+\rho_{5} A_{5} \partial u_{5}+u_{5} A_{5} \partial \rho_{5} & =0 \\
A_{5} \partial p+\rho_{5} u_{5} A_{5} \partial u_{5} & =0 \\
u_{5} \partial u_{5}+C_{p_{5}} \partial T_{5} & =0 \\
\frac{1}{p} \partial p-\frac{1}{T_{5}} \partial T_{5}-\frac{1}{\rho_{5}} \partial \rho_{5} & =0
\end{aligned}
$$

Stream 8 is defined by

$$
\begin{aligned}
\rho_{8} u_{8} \partial A_{8}+\rho_{8} A_{8} \partial u_{8}+u_{8} A_{8} \partial \rho_{8}+\partial \dot{m}_{8} & =0 \\
A_{8} \partial p+\rho_{8} u_{8} A_{8} \partial u_{8}-u_{8} \partial \dot{m}_{8} & =0 \\
u_{8} \partial u_{8}+C_{p_{8}} \partial T_{8} & =0 \\
\frac{1}{p} \partial p-\frac{1}{T_{8}} \partial T_{8}-\frac{1}{\rho_{8}} \partial \rho_{8} & =0
\end{aligned}
$$

Stream 9 is defined by

$$
\begin{array}{r}
\rho_{9} u_{9} \partial A_{9}+\rho_{9} A_{9} \partial u_{9}+u_{9} A_{9} \partial \rho_{9}+\partial \dot{m}_{9}=0 \\
A_{9} \partial p+\rho_{9} u_{9} A_{9} \partial u_{9}-u_{9} \partial \dot{m}_{9}=0 \\
u_{9} \partial u_{9}+C_{p_{9}} \partial T_{9}=0
\end{array}
$$




$$
\frac{1}{p} \partial p-\frac{1}{T_{9}} \partial T_{9}-\frac{1}{\rho_{9}} \partial \rho_{9}=0
$$

Stream 10 is defined by

$$
\begin{array}{r}
\rho_{10} u_{10} \partial A_{10}+\rho_{10} A_{10} \partial u_{10}+u_{10} A_{10} \partial \rho_{10}-\partial \dot{m}_{10}=0 \\
A_{10} \partial p+\rho_{10} u_{10} A_{10} \partial u_{10}+\left(u_{10}-u_{2}\right) \partial \dot{m}_{2,10}+\left(u_{10}-u_{8}\right) \partial \dot{m}_{8}=0 \\
u_{10} \partial u_{10}+C_{p_{10}} \partial T_{10}+\frac{\left\{C_{p_{2}}\left(T_{10}-T_{2}\right)+\frac{1}{2} u_{10}^{2}-\frac{1}{2} u_{2}^{2}\right\} \partial \dot{m}_{2,10}}{\dot{m}_{10}} \\
+\frac{\left\{C_{p_{8}}\left(T_{10}-T_{8}\right)+\frac{1}{2} u_{10}^{2}-\frac{1}{2} u_{8}^{2}\right\} \partial \dot{m}_{8}}{\dot{m}_{10}}=0 \\
\frac{1}{p} \partial p-\frac{1}{T_{10}} \partial T_{10}-\frac{1}{\rho_{10}} \partial \rho_{10}=0
\end{array}
$$

Stream 11 is defined by

$$
\begin{array}{r}
\rho_{11} u_{11} \partial A_{11}+\rho_{11} A_{11} \partial u_{11}+u_{11} A_{11} \partial \rho_{11}-\partial \dot{m}_{11}=0 \\
A_{11} \partial p+\rho_{11} u_{11} A_{11} \partial u_{11}+\left(u_{11}-u_{3}\right) \partial \dot{m}_{3,11}+\left(u_{11}-u_{9}\right) \partial \dot{m}_{9}=0 \\
u_{11} \partial u_{11}+C_{p_{11}} \partial T_{11}+\frac{\left\{C_{p_{3}}\left(T_{11}-T_{3}\right)+\frac{1}{2} u_{11}^{2}-\frac{1}{2} u_{3}^{2}\right\} \partial \dot{m}_{3,11}}{\dot{m}_{11}} \\
+\frac{\left\{C_{p_{9}}\left(T_{11}-T_{9}\right)+\frac{1}{2} u_{11}^{2}-\frac{1}{2} u_{9}^{2}\right\} \partial \dot{m}_{9}}{\dot{m}_{11}}-\partial h_{p r_{11}}=0 \\
\frac{1}{p} \partial p-\frac{1}{T_{11}} \partial T_{11}-\frac{1}{\rho_{11}} \partial \rho_{11}=0
\end{array}
$$

Summation of the areas gives

$$
\partial A_{1}+\partial A_{2}+\partial A_{3}+\partial A_{4}+\partial A_{5}+\partial A_{8}+\partial A_{8}+\partial A_{10}+\partial A_{11}-\partial A=0
$$




\section{C.2 Entrainment Model}

Once again, the duct was broken into three sections. These sections were the same regions described in Section C.1.

In general, the mass flow in any stream can be defined as

$$
\dot{m}=\rho Q
$$

where $\mathrm{Q}$ is the volumetric flow rate. Writing Equation C.67 in differential form

$$
\partial \dot{m}=\rho \partial Q+Q \partial \rho
$$

and substituting Equation 4.10

$$
\partial \dot{m}=c\left(\frac{1-r}{1+r}\right) U_{2} \rho+c\left(\frac{1-r}{1+r}\right) U_{2} x \partial \rho
$$

where, as before, $U_{2}$ is the high speed stream inlet velocity, $\mathrm{r}$ is the velocity ratio, and $\mathrm{c}$ is a proportionality constant. This proportionality constant was the value changed when the mixing rate was varied in the control volume model.

\section{C.2.1 Sections 1 and 2}

The mass flows entrained into and out of the various streams in sections 1 and 2 of the duct can be defined as follows.

$$
\begin{array}{r}
\partial \dot{m}_{1}=c_{1}\left(\frac{1-r_{1}}{1+r_{1}}\right) U_{2} \rho_{1}+c_{1}\left(\frac{1-r_{1}}{1+r_{1}}\right) U_{2} x \partial \rho_{1} \\
\partial \dot{m}_{2,1}=\frac{E_{m_{1}}}{1+E_{m_{1}}} \partial \dot{m}_{1}
\end{array}
$$




$$
\begin{aligned}
& \partial \dot{m}_{3,1}=\frac{1}{1+E_{m_{1}}} \partial \dot{m}_{1} \\
& \partial \dot{m}_{2,4}=\frac{E_{m_{4}}}{1+E_{m_{4}}} \partial \dot{m}_{4} \\
& \partial \dot{m}_{3,5}=\frac{E_{m_{5}}}{1+E_{m_{5}}} \partial \dot{m}_{5} \\
& \partial \dot{m}_{4}=c_{4}\left(\frac{1-r_{4}}{1+r_{4}}\right) U_{2} \rho_{4}+c_{4}\left(\frac{1-r_{4}}{1+r_{4}}\right) U_{2} x \partial \rho_{4} \\
& \partial \dot{m}_{5}=c_{5}\left(\frac{1-r_{5}}{1+r_{5}}\right) U_{3} \rho_{5}+c_{5}\left(\frac{1-r_{5}}{1+r_{5}}\right) U_{3} x \partial \rho_{5} \\
& \partial \dot{m}_{6}=\frac{1}{1+E_{m_{4}}} \partial \dot{m}_{4} \\
& \partial \dot{m}_{7}=\frac{1}{1+E_{m_{5}}} \partial \dot{m}_{5}
\end{aligned}
$$

where $U_{2}$ and $U_{3}$ are the inlet velocities of streams 2 and 3 respectively. The velocity ratios are defined as

$$
\begin{aligned}
& r_{1}=\frac{u_{3}}{u_{2}} \\
& r_{4}=\frac{u_{6}}{u_{2}} \\
& r_{5}=\frac{u_{7}}{u_{3}}
\end{aligned}
$$

and $E_{m_{1}}, E_{m_{4}}$, and $E_{m_{5}}$ are the mass entrainment ratios, defined by Hermanson and Dimotakis[10], and given by

$$
\begin{aligned}
& E_{m_{1}}=\frac{\partial \dot{m}_{2,1}}{\partial \dot{m}_{3,1}}=\left(\frac{\rho_{3}}{\rho_{2}}\right)^{-\frac{1}{2}}\left\{1+0.68\left(\frac{1-r_{1}}{1+r_{1}}\right)\right\} \\
& E_{m_{4}}=\frac{\partial \dot{m}_{2,4}}{\partial \dot{m}_{6}}=\left(\frac{\rho_{6}}{\rho_{2}}\right)^{-\frac{1}{2}}\left\{1+0.68\left(\frac{1-r_{4}}{1+r_{4}}\right)\right\} \\
& E_{m_{5}}=\frac{\partial \dot{m}_{3,5}}{\partial \dot{m}_{7}}=\left(\frac{\rho_{7}}{\rho_{3}}\right)^{-\frac{1}{2}}\left\{1+0.68\left(\frac{1-r_{5}}{1+r_{5}}\right)\right\}
\end{aligned}
$$




\section{C.2.2 Section3}

The entrained mass flows in section 3 are defined by

$$
\begin{aligned}
\partial \dot{m}_{1}=c_{1}\left(\frac{1-r_{1}}{1+r_{1}}\right) U_{2} \rho_{1}+c_{1}\left(\frac{1-r_{1}}{1+r_{1}}\right) U_{2} x \partial \rho_{1} \\
\partial \dot{m}_{2,1}=\frac{E_{m_{1}}}{1+E_{m_{1}}} \partial \dot{m}_{1} \\
\partial \dot{m}_{3,1}=\frac{1}{1+E_{m_{1}}} \partial \dot{m}_{1} \\
\partial \dot{m}_{2,10}=\frac{E_{m_{10}}}{1+E_{m_{10}}} \partial \dot{m}_{10} \\
\partial \dot{m}_{3,11}=\frac{E_{m_{11}}}{1+E_{m_{11}}} \partial \dot{m}_{11} \\
\partial \dot{m}_{10}=c_{10}\left(\frac{1-r_{10}}{1+r_{10}}\right) U_{2} \rho_{10}+c_{10}\left(\frac{1-r_{10}}{1+r_{10}}\right) U_{2} x \partial \rho_{10} \\
\partial \dot{m}_{11}=c_{11}\left(\frac{1-r_{11}}{1+r_{11}}\right) U_{3} \rho_{11}+c_{11}\left(\frac{1-r_{11}}{1+r_{11}}\right) U_{3} x \partial \rho_{11} \\
\partial \dot{m}_{8}=\frac{1}{1+E_{m_{10}}} \partial \dot{m}_{10} \\
\partial \dot{m}_{9}=\frac{1}{1+E_{m_{11}}} \partial \dot{m}_{11}
\end{aligned}
$$

where the velocity ratios are defined as

$$
\begin{aligned}
& r_{10}=\frac{u_{8}}{u_{2}} \\
& r_{11}=\frac{u_{9}}{u_{3}}
\end{aligned}
$$

and $E_{m_{10}}$ and $E_{m_{11}}$ are the mass entrainment ratios given by

$$
\begin{aligned}
& E_{m_{10}}=\frac{\partial \dot{m}_{2,10}}{\partial \dot{m}_{8}}=\left(\frac{\rho_{8}}{\rho_{2}}\right)^{-\frac{1}{2}}\left\{1+0.68\left(\frac{1-r_{10}}{1+r_{10}}\right)\right\} \\
& E_{m_{11}}=\frac{\partial \dot{m}_{3,11}}{\partial \dot{m}_{9}}=\left(\frac{\rho_{9}}{\rho_{3}}\right)^{-\frac{1}{2}}\left\{1+0.68\left(\frac{1-r_{11}}{1+r_{11}}\right)\right\}
\end{aligned}
$$




\section{C.3 Combustion Chemistry}

As noted in Section 3.1 the reaction mechanism used for the hydrogen-air reaction was

$$
\left(H_{2}+\frac{1}{\alpha} N_{2}\right)+\frac{1}{2 \psi}\left(O_{2}+3.76 N_{2}\right) \rightarrow H_{2} O+\left(\frac{1}{2 \psi}-\frac{1}{2}\right) O_{2}+\left(\frac{1.88}{\psi}+\frac{1}{\alpha}\right) N_{2}
$$

This can be written for streams 1,5 , and 11 where reaction occurs. These reaction mechanisms are

$$
\begin{aligned}
\left(H_{2}+\frac{1}{\alpha_{1}} N_{2}\right)+\frac{1}{2 \psi_{1}}\left(O_{2}+3.76 N_{2}\right) & \rightarrow H_{2} O \\
& +\left(\frac{1}{2 \psi_{1}}-\frac{1}{2}\right) O_{2}+\left(\frac{1.88}{\psi_{1}}+\frac{1}{\alpha_{1}}\right) N_{2} \\
\left(H_{2}+\frac{1}{\alpha_{5}} N_{2}\right)+\frac{1}{2 \psi_{5}}\left(O_{2}+3.76 N_{2}\right) & \rightarrow H_{2} O \\
& +\left(\frac{1}{2 \psi_{5}}-\frac{1}{2}\right) O_{2}+\left(\frac{1.88}{\psi_{5}}+\frac{1}{\alpha_{5}}\right) N_{2} \\
\left(H_{2}+\frac{1}{\alpha_{11}} N_{2}\right)+\frac{1}{2 \psi_{11}}\left(O_{2}+3.76 N_{2}\right) & \rightarrow H_{2} O \\
+ & \left(\frac{1}{2 \psi_{11}}-\frac{1}{2}\right) O_{2}+\left(\frac{1.88}{\psi_{11}}+\frac{1}{\alpha_{11}}\right) N_{2}
\end{aligned}
$$

where $\alpha, \phi$, and $\psi$ are defined for the three streams as follows

$$
\begin{aligned}
\alpha_{1} & =\left.\frac{N_{H_{2}}}{N_{N_{2}}}\right|_{s t r e a m 3} \\
\alpha_{5} & =\left.\frac{N_{H_{2}}}{N_{N_{2}}}\right|_{s t r e a m 5} \\
\alpha_{11} & =\left.\frac{N_{H_{2}}}{N_{N_{2}}}\right|_{s t r e a m 11}
\end{aligned}
$$

where $\mathrm{N}$ is the number of moles of a given gas, 


$$
\begin{gathered}
\Phi_{1}=\frac{\left.N_{H_{2}}\right|_{\text {stream } 3}}{\left.2 N_{O_{2}}\right|_{\text {stream } 2}} \\
\Phi_{5}=\frac{\left.N_{H_{2}}\right|_{\text {stream } 3}}{\left.2 N_{O_{2}}\right|_{\text {stream } 7}} \\
\Phi_{11}=\frac{\left.N_{H_{2}}\right|_{\text {stream } 3}}{\left.2 N_{O_{2}}\right|_{\text {stream }} 9} \\
\psi_{1}=\Phi_{1} \frac{\partial \dot{m}_{3,1}}{\partial \dot{m}_{2,1}} \frac{W_{2}}{W_{3}} \frac{\left.X_{H_{2}}\right|_{\text {stream } 3}}{\left.X_{O_{2}}\right|_{\text {stream } 2}} \\
\psi_{5}=\Phi_{5} \frac{\partial \dot{m}_{3,5}}{\partial \dot{m}_{7}} \frac{W_{7}}{W_{3}} \frac{\left.X_{H_{2}}\right|_{\text {stream } 3}}{\left.X_{O_{2}}\right|_{\text {stream } 7}} \\
\psi_{11}=\Phi_{11} \frac{\partial \dot{m}_{3,11}}{\partial \dot{m}_{9}} \frac{W_{9}}{W_{3}} \frac{\left.X_{H_{2}}\right|_{\text {stream } 3}}{\left.X_{O_{2}}\right|_{\text {stream }} 9}
\end{gathered}
$$

where $\mathrm{W}$ is the molecular weight of a given stream and $\mathrm{X}$ is the mole fraction of a given gas in a given stream.

The heat release due to reaction is given by $\partial h_{p r_{1}}, \partial h_{p r_{5}}$, and $\partial h_{p r_{11}}$ in Equations C.3, C.19, C.32, and C.64. These are computed using

$$
\partial h_{p r, k}=\sum-\nu_{k}\left[\int_{T_{0}}^{T} C_{p, k} \partial T+\Delta h_{f, k}^{\circ}\right]
$$

where $\Delta h_{f}^{\circ}$ is the enthalpy of formation referenced at $T_{0}=298 \mathrm{~K}$ and $\nu$ is the stoichiometric coefficient for each species, negative in value for reactants and positive for products.

$$
2556-37
$$

